Article

\title{
Algebra of Complex Vectors and Applications in Electromagnetic Theory and Quantum Mechanics
}

\author{
Kundeti Muralidhar \\ Physics Department, National Defence Academy, Khadakwasla, Pune-411 023, India; \\ E-Mail: kundetimuralidhar@gmail.com \\ External Editor: Palle E.T. Jorgensen
}

Received: 14 March 2015 / Accepted: 14 August 2015 / Published: 20 August 2015

\begin{abstract}
A complex vector is a sum of a vector and a bivector and forms a natural extension of a vector. The complex vectors have certain special geometric properties and considered as algebraic entities. These represent rotations along with specified orientation and direction in space. It has been shown that the association of complex vector with its conjugate generates complex vector space and the corresponding basis elements defined from the complex vector and its conjugate form a closed complex four dimensional linear space. The complexification process in complex vector space allows the generation of higher $n$-dimensional geometric algebra from $(n-1)$-dimensional algebra by considering the unit pseudoscalar identification with square root of minus one. The spacetime algebra can be generated from the geometric algebra by considering a vector equal to square root of plus one. The applications of complex vector algebra are discussed mainly in the electromagnetic theory and in the dynamics of an elementary particle with extended structure. Complex vector formalism simplifies the expressions and elucidates geometrical understanding of the basic concepts. The analysis shows that the existence of spin transforms a classical oscillator into a quantum oscillator. In conclusion the classical mechanics combined with zeropoint field leads to quantum mechanics.
\end{abstract}

Keywords: geometric algebra; complex vectors; particle spin; zeropoint field

\section{Introduction}

The ideas and concepts of physics are well expressed in the language of mathematics. The language of mathematics is improved from time to time to express new ideas. In the history of development of 
mathematics; complex algebra was developed from the real algebra, quaternion algebra was developed from complex algebra, vector algebra was developed from quaternion algebra and vector algebra was the basis for matrix algebra, tensor algebra and vector calculus. In 1880, Willard J Gibbs invented vector algebra [1] building upon the work of Hamilton. Certain limitations of vector algebra were effectively addressed in Clifford algebra by introducing a geometric product, a canonical composition of inner and outer products. A cross product in vector algebra was replaced by a better product called outer product which gives both rotation and the planar nature of the product. Clifford algebra or Geometric algebra is more efficient than the matrix algebra because of the fact that the components of geometric algebra can be expressed without introducing any arbitrary basis and turned out to be a superior mathematical tool to express many of the physical concepts and proved to provide simpler and straightforward description to the mathematical and physical problems. Clifford showed that the vector algebra is the subalgebra of geometric algebra. However, for several decades the Clifford algebra was lost amongst the wealth of vector algebra. Only much later, because of nature's violation of parity symmetry, we make a distinction between vectors and pseudovectors or axialvectors and between scalars and pseudoscalars which are necessary for writing effective Hamiltonian for weak interaction. But the geometric algebra contains bivectors and trivectors built into its foundations and these correspond directly to pseudovectors and pseudoscalars. The Clifford algebra was rediscovered by Dirac in his relativistic theory of electron [2] and introduced a complex operator $\gamma_{\mu} \partial^{\mu}$, where $\gamma$-matrices satisfy the anti-commutation relations. These matrices have been thought of as an internal electron spin space. The geometric algebra was rediscovered again by Hestenes [3-5] in the 1960s and it is being used by a growing number of physicists today. A wider application of geometric algebra came into existence with the idea of Hestenes that the Dirac matrices could be manipulated as vectors in spacetime algebra. In recent developments of mathematics, Sobczyk [6] introduced unitary geometric algebra which deals with complex vectors.

The rotation in vector algebra has two serious problems. Firstly the physical quantities change differently under space inversion, a reflection through a single chosen point. The displacements and velocities are equal to negative of the corresponding noninverted quantities. Thus quantities like angular velocity, torque remain same under space inversion. In vector algebra, this is handled with an ad-hoc introduction of an axialvector. These are simply decreed to be vectors that do not change sign upon space inversion of the corresponding physical systems. Secondly the cross product exists only in three dimensions and in four dimensions the concept of a vector orthogonal to the plane defined by a pair vectors is not unique. Thus rotations in four dimensional spacetime cannot be uniquely defined. Further, in two dimensional mechanics to represent quantities like angular velocity in vector algebra, one must somehow allow a vector perpendicular to a plane. Such limitations are simply removed by introducing a bivector product. The standard tensor algebra has certain practical limitations like its inability to deal with spinors. The tensor algebra is a coordinate based algebra and therefore so much of mathematical labor is required for proving covariance of physical quantities. However, geometric algebra simplifies and unifies mathematical methods in classical physics and non relativistic quantum mechanics.

The outer product was first introduced by Grassmann in 1880. To develop inner and outer products in a systematic way, first consider a square of the sum of two vectors a and b. A sum of two vectors is simply another vector and its square must be a scalar. This is expressed in the following manner.

$$
(\mathbf{a}+\mathbf{b})^{2}=a^{2}+b^{2}+(\mathbf{a b}+\mathbf{b a})
$$


As the term $\mathbf{a b}+\mathbf{b a}$ must be a scalar, the symmetric product or scalar product is then defined as

$$
\mathbf{a} \cdot \mathbf{b}=\frac{1}{2}(\mathbf{a b}+\mathbf{b a})
$$

In the above expression, we are assuming the symmetry of the product $\mathbf{a b}=\mathbf{b a}$ or $\mathbf{a b}-\mathbf{b a}=0$ or in other words the vectors $\mathbf{a}$ and $\mathbf{b}$ commute. In the general case the products $\mathbf{a b}$ and $\mathbf{b a}$ are not equal. Most probably by ignoring this simple fact, over hundred years the existence of the so called the Grassmann product is not reflected in the entire field of vector algebra. To find this product, let us take the product of vectors $(\mathbf{a}-\mathbf{b})$ and $(\mathbf{a}+\mathbf{b})$.

$$
(\mathbf{a}-\mathbf{b})(\mathbf{a}+\mathbf{b})=a^{2}-b^{2}+(\mathbf{a b}-\mathbf{b a})
$$

Since the vectors $(\mathbf{a}-\mathbf{b})$ and $(\mathbf{a}+\mathbf{b})$ are not collinear, their product cannot be a scalar. From Equation (3) we see that $a^{2}-b^{2}$ is a scalar and ( $\mathbf{a b}-\mathbf{b a}$ ) must represent a plane containing both the vectors $\mathbf{a}$ and $\mathbf{b}$. Now, we are in a position to define a new product

$$
\mathbf{a} \wedge \mathbf{b}=\frac{1}{2}(\mathbf{a b}-\mathbf{b a}),
$$

where the symbol $\wedge$ is pronounced as wedge. Thus the outer product can be obtained by introducing an asymmetric product by taking $\mathbf{a b}=-\mathbf{b a}$ or $\mathbf{a b}+\mathbf{b a}=0$ or in other words the vectors $\mathbf{a}$ and $\mathbf{b}$ anticommute. This product represents an oriented plane with magnitude. The product $\mathbf{a} \wedge \mathbf{b}$ is called a bivector and hence the outer product is also called a bivector product. Now, we write Equation (2) in the following form

$$
\mathbf{a} \cdot \mathbf{b}=\frac{1}{2}(\mathbf{a b}+\mathbf{b a})=\mathbf{a b}-\frac{1}{2}(\mathbf{a b}-\mathbf{b a})=\mathbf{a b}-\mathbf{a} \wedge \mathbf{b} .
$$

Rearranging the above equation, we find the geometric product of two vectors $\mathbf{a}$ and $\mathbf{b}$.

$$
\mathbf{a b}=\mathbf{a} \cdot \mathbf{b}+\mathbf{a} \wedge \mathbf{b}
$$

Thus the geometric product of two vectors is equal to a sum of scalar product and bivector product of the corresponding vectors. Changing the order of vectors in a geometric product is called reversion operation and it is denoted by the symbol over bar.

$$
\begin{gathered}
\overline{\mathbf{a b}}=\mathbf{b a} \\
\mathbf{b a}=\mathbf{a} \cdot \mathbf{b}-\mathbf{a} \wedge \mathbf{b} .
\end{gathered}
$$

The essential feature of a geometric product is that it is a sum of two different types of objects; a scalar and a bivector. Further these products are symmetric and asymmetric components of the geometric product. It can be seen that the bivector product is invariant under space inversion. Unlike in vector algebra, in geometric algebra the quantities such as rotations and angular velocity are correctly represented by bivectors because the bivectors are unchanged under space inversion. A single vector is used to represent all the information to abstract from a single curve by approximating with very small straight vectors. Similarly, a bivector physical surface can be represented by approximating with many small bivectors. It is independent of all details of the path of the actual rotation. The bivetor in a way represents a physical rotation that depends only on orientation of the plane of direct rotation from the 
initial to final attitude on the total magnitude of the direct rotation. A product of three orthogonal vectors is called a trivector which represents an oriented volume in space. If $\mathbf{a}, \mathbf{b}$ and $\mathbf{c}$ are three orthogonal vectors, then the product of these vectors can be expressed as a scalar times a pseudoscalar, $\mathbf{a b c}=\mathbf{i} \alpha$. The pseudoscalar is the product of three orthonormal unit vectors along $\mathbf{a}, \mathbf{b}$ and $\mathbf{c}$ and represents a unit oriented volume. Geometric algebra is a graded algebra. The scalar is a grade zero element and we call it a 0 -vector, a vector grade is one ( 1 -vector), a bivector grade is two (2-vector) and in general an element with grade $\mathrm{k}$ is called k-vector. Thus the geometric algebra includes scalar, vector, bivector and other higher grade vectors. The elements of this algebra are called multivectors and form a linear space. Thus the mathematics developed for geometric product can be viewed as geometric algebra. In fact the geometric product can be defined in any dimensional space. The starting point for geometric algebra is the real vector space from which the entire algebra will be generated. The space of elements of geometric algebra or multivectors is linear over the real numbers. It means, if $\alpha$ and $\beta$ are scalars and $\mathrm{A}$ and $\mathrm{B}$ are multivectors then $\alpha A+\beta B$ is also a multivector and hence multivecotrs form a linear space. Any multivector can be written as a sum of geometric products of vectors. An r-vector is a product of $r$ orthogonal vectors with grade $r$. A vector of grade $r$ is even (odd) when its grade is even (odd). Thus scalars and bivectors are of even grade and vectors and trivectors are of odd grade. The inner product of vectors $A_{k}$ of grade $k$ and $B_{m}$ of grade $m$ is defined as

$$
A_{k} \cdot B_{m}=\frac{1}{2}\left[A_{k} B_{m}+(-1)^{|k-m|} B_{m} A_{k}\right] .
$$

Similarly the outer product satisfies

$$
A_{k} \wedge B_{m}=\frac{1}{2}\left[A_{k} B_{m}-(-1)^{|k-m|} B_{m} A_{k}\right] .
$$

Then the geometric product of vectors $A_{k}$ of grade $k$ and $B_{m}$ of grade $m$ is defined as

$$
A_{k} B_{m}=A_{k} \cdot B_{m}+A_{k} \wedge B_{m} .
$$

Detailed account of geometric algebra was discussed in many books, particularly by Hestenes [7] and Doran and Lasenby [8].

In this article, the imaginary vector introduced by Gibbs has been reformulated by replacing the unit imaginary by pseudoscalar. Then the complex vector is defined as a sum of a vector and a bivector, and found to be a natural extension of a vector. The complex vector is expressed as $Z=\mathbf{a}+\mathbf{i} \mathbf{b}$. The complex vector $Z$ along with its conjugate $\bar{Z}$ generate a complex vector space. The complexification process in complex vector space allows the generation of higher $n$ dimensional geometric algebra $\mathcal{G}(n)$ from $(n-1)$ dimensional algebra $\mathcal{G}(n-1)$ by considering the unit pseudoscalar identification with square root of minus one. A quaternion can be defined from the set of basis bivectors and such quaternions form four dimensional algebra. The spacetime algebra of four dimensional spacetime $\mathcal{G}(1,3)$ can be generated from the geometric algebra $\mathcal{G}(3)$ by considering a vector identification with square root of plus one. A higer dimensional complex spacetime $\mathcal{G}(1, n+1)$ can be generated from $\mathcal{G}(1, n)$ by considering a unit pseudoscalar of $(n+1)$ dimensions. The complex vector algebra and the generation of complex vector space and spacetime are presented in Section 2.

It is well known that the elliptic harmonic motion can be better visualized in the complex vector approach and complex vector methods can be easily incorporated into the electromagnetic theory. In a 
finite transverse extent of an electromagnetic wave, the electromagnetic field lines are closed loops and represent circulating energy flow in addition to the energy flow in the propagation direction. Considering this view, we have expressed the photon energy as a multivector and explored the internal field rotations or oscillations in the complex vector formalism. In the microcosm, particles are treated as oscillators and hence their paths are blurred. The entire quantum mechanics was developed based on the fact that the particles are associated with waves or their positions and momenta are not exactly determined. The ineternal oscillatory behavior of particles has been found to be due to the presence of fluctuating electromagnetic zeropoint field. In the complex vector space such oscillations of the particles are considered as spatial local rotations and the angular momentum corresponding to these rotations is the zeropoint angular momentum or particle spin. The particles are in general not considered as point particles but with internal rotation as its structure. The superposition of internal rotation and translational motion of a particle can be expressed in terms of complex vector representing the particle complex position and complex momentum. The dynamics of a particle with extended structure and the particle internal harmonic oscillator in the complex vector formalism are developed. Section 3 is devoted to the applications of complex vector algebra in the electromagnetic theory and dynamics of the particles with extended structure. A short account of the algebra of complex vectors and its applications in electromagnetic theory and quantum mechanics is presented in the summary.

\section{Algebra of Complex Vectors}

The idea of complex vector has its origin in the formulation of elliptic harmonic motion was described by Gibbs and he called these as imaginary vectors or bivectors [1]. An imaginary vector is defined as a sum of two real vectors of which one has been multiplied by the imaginary scalar or unit imaginary $i=\sqrt{-1}$. An imaginary vector $A$ is then defined as

$$
A=\mathbf{a}+i \mathbf{b}
$$

Here, $\mathbf{a}$ and $\mathbf{b}$ are real vectors. From the above definition, Gibbs stated that the imaginary vector may be regarded as an inevitable extension of a real vector. This form of complex vector has been widely used as a Faraday bivector to represent electromagnetic fields, and to study polarization of the electromagnetic field $[9,10]$. However, considering the property of pseudoscalar $\mathbf{i}^{2}=-1$, we reformulate the imaginary vector in geometric algebra by replacing the unit imaginary by pseudoscalar and the resulting complex vector is defined as a sum of a vector and a bivector in three dimensions.

$$
Z=\mathbf{a}+\mathbf{i} \mathbf{b}
$$

The advantage of this definition of complex vector is that it gives additional geometric understanding of orientation of plane of rotation in space. We define a complex scalar as a sum of a scalar and a trivector and it can be expressed as $z=\alpha+\mathbf{i} \beta$ which is analogous to a complex number. A reversion operation on a complex vector changes the sign of bivector.

$$
\bar{Z}=\mathbf{a}-\mathbf{i} \mathbf{b}
$$


The complex vector $\bar{Z}$ is known as complex conjugate of $Z$. Two complex vectors are equal when their vector and bivector parts are equal. The inner product of two complex vectors $Z=\mathbf{a}+\mathbf{i} \mathbf{b}$ and $Y=\mathbf{c}+\mathbf{i} \mathbf{d}$ can be expressed as

$$
Z . Y=(\mathbf{a} . \mathbf{c}-\mathbf{b . d})+\mathbf{i}(\mathbf{b . c}+\mathbf{a . d})=\alpha+\mathbf{i} \beta .
$$

Thus the scalar product of two complex vectors is a complex scalar. The outer product of complex vectors $Z$ and $Y$ is

$$
Z \wedge Y=(\mathbf{a} \wedge \mathbf{c}-\mathbf{b} \wedge \mathbf{d})+\mathbf{i}(\mathbf{b} \wedge \mathbf{c}+\mathbf{a} \wedge \mathbf{d})
$$

The term $(\mathbf{a} \wedge \mathbf{c}-\mathbf{b} \wedge \mathbf{d})$ is a bivector and the term $\mathbf{i}(\mathbf{b} \wedge \mathbf{c}+\mathbf{a} \wedge \mathbf{d})$ is vector. Thus the bivector product of two complex vectors is a complex vector. From the above two products one can see that the geometric product of two complex vectors is a combination of a scalar, vector, bivector and trivector parts. Thus the geometric product of two complex vectors is a multivector. The inner and outer products of complex vectors are in general known as symmetric and asymmetric products respectively [11]. Multiplying a complex vector by a complex scalar does not change the form of complex vector.

$$
Z z=(\mathbf{a}+\mathbf{i} \mathbf{b})(\alpha+\mathbf{i} \beta)=(\alpha \mathbf{a}-\beta \mathbf{b})+\mathbf{i}(\alpha \mathbf{b}+\beta \mathbf{a})
$$

If we express the complex scalar as a rotor, $z=e^{\mathbf{i} \theta}$, then it can be seen that the multiplication of a complex vector by a complex scalar rotates the complex vector in the plane ab by an angle $\theta$. Two complex vectors $Z=\mathbf{a}+\mathbf{i} \mathbf{b}$ and $Y=\mathbf{c}+\mathbf{i} \mathbf{d}$ are said to be perpendicular when the condition a.c $=0$ is satisfied and when they are parallel when the condition $\mathbf{a} \wedge \mathbf{c}=0$ is satisfied. The square of a complex vector is a complex scalar.

$$
Z^{2}=(\mathbf{a}+\mathbf{i} \mathbf{b})(\mathbf{a}+\mathbf{i} \mathbf{b})=a^{2}-b^{2}+2 \mathbf{i}(\mathbf{a} \cdot \mathbf{b})
$$

Consider that the vectors $\mathbf{a}$ and $\mathbf{b}$ are orthogonal to each other then we have $\mathbf{a} \cdot \mathbf{b}=0$. In this case the complex vector represents an oriented directional ellipse. The bivector $\mathbf{i} b$ represents an oriented plane and the vector $\mathbf{a}$ lies in the plane of $\mathbf{i} \mathbf{b}$. The rotation in the plane $\mathbf{i} \mathbf{b}$ is counterclockwise for the complex vector $Z=\mathbf{a}+\mathbf{i} \mathbf{b}$ and clockwise for $\bar{Z}$. Since the vectors $\mathbf{a}$ and $\mathbf{b}$ are orthogonal, the square of $\mathbf{a}$ complex vector is a scalar.

$$
Z^{2}=\bar{Z}^{2}=a^{2}-b^{2}
$$

The product of a complex vector with its conjugate contains a scalar and a vector parts. The products $Z \bar{Z}$ and $\bar{Z} Z$ are written as

$$
\begin{aligned}
& Z \bar{Z}=a^{2}+b^{2}-2 \mathbf{i}(\mathbf{a} \wedge \mathbf{b}), \\
& \bar{Z} Z=a^{2}+b^{2}+2 \mathbf{i}(\mathbf{a} \wedge \mathbf{b}) .
\end{aligned}
$$

Since, $\mathbf{i}$ is a pseudoscalar which commutes with all vectors in three dimensional space, the quantity $2 \mathbf{i}(\mathbf{a} \wedge \mathbf{b})$ is a vector and it is normal to the orientation of the bivector $\mathbf{a} \wedge \mathbf{b}$. The scalar part is equal to the scalar product of $\bar{Z}$ and $Z$.

$$
\bar{Z} \cdot Z=\frac{1}{2}(\bar{Z} Z+Z \bar{Z})=a^{2}+b^{2}
$$

The vector part is equal to the bivector product of $\bar{Z}$ and $Z$.

$$
\bar{Z} \wedge Z=\frac{1}{2}(\bar{Z} Z-Z \bar{Z})=2 \mathbf{i}(\mathbf{a} \wedge \mathbf{b})
$$


In the case when the magnitudes of vectors $\mathbf{a}$ and $\mathbf{b}$ are equal, then the complex vector represents an oriented directional circle. Then the square of complex vector is $Z^{2}=\bar{Z}^{2}=0$ and therefore in this case the complex vector may be called complex null vector. In spacetime algebra, a complex vector is defined as a combination of relative vector and spatial bivector. We shall study these complex vectors in the next section. In n-dimensional space, one can define a complex vector as a sum of k-grade vector and a product of pseudoscalar times another k-grade vector. A complex vector of k-grade is defined as

$$
C_{k}=\mathbf{a}_{k}+\mathbf{i}_{n} \mathbf{b}_{k}
$$

The inner product of a vector $\mathbf{d}$ and a k-grade complex vector $C_{k}$ is defined for odd $k \geq 1$ as

$$
\begin{aligned}
& \text { d. } C_{k}=\left[\frac{1}{2}\left(\mathbf{d} \cdot C_{k}+\bar{C}_{k} \cdot \mathbf{d}\right)\right]_{k-1}, \\
& \text { d. } C_{k+1}=\left[\frac{1}{2}\left(\mathbf{d} \cdot C_{k}-\bar{C}_{k} \cdot \mathbf{d}\right)\right]_{k} .
\end{aligned}
$$

Similarly, the outer product of vector $\mathbf{d}$ and a k-grade complex vector $C_{k}$ is defined for odd $k \geq 1$ as

$$
\begin{gathered}
\mathbf{d} \wedge C_{k}=\left[\frac{1}{2}\left(\mathbf{d} \cdot C_{k}-\bar{C}_{k} \cdot \mathbf{d}\right)\right]_{k+1}, \\
\mathbf{d} \wedge C_{k+1}=\left[\frac{1}{2}\left(\mathbf{d} \cdot C_{k}+\bar{C}_{k} \cdot \mathbf{d}\right)\right]_{k+2} .
\end{gathered}
$$

Further, a detailed account of complex vectors including products of trivectors has been given by Sobczyk [6].

\subsection{Complex Vector Space}

A short version of complex vector space has been developed by the author in the reference [12] and a complete account of which is presented in this section. The complex vectors $Z=\mathbf{a}+\mathbf{i} \mathbf{b}$ and its conjugate $\bar{Z}=\mathbf{a}-\mathbf{i} \mathbf{b}$ defined in the Equations (13) and (14) actually form a physical space when the third direction is chosen normal to the plane containing the orthogonal vetors $\mathbf{a}$ and $\mathbf{b}$. Let $\mathbf{c}=2 \mathbf{i}(\mathbf{a} \wedge \mathbf{b})$ be a vector normal to both $\mathbf{a}$ and $\mathbf{b}$. Now, the vectors $\mathbf{a}, \mathbf{b}$ and $\mathbf{c}$ can be expressed in terms of $Z$ and $\bar{Z}$ complex vectors in the following form.

$$
\begin{gathered}
\mathbf{a}=\frac{Z+\bar{Z}}{2} \\
\mathbf{b}=\frac{Z-\bar{Z}}{2 \mathbf{i}} \\
\mathbf{c}=\frac{Z \bar{Z}-\bar{Z} Z}{2}
\end{gathered}
$$

If we choose a set of orthonormal right handed unit vectors $\left\{\boldsymbol{\sigma}_{k} ; k=1,2,3\right\}$ along the directions of orthogonal vectors $\mathbf{a}, \mathbf{b}$ and $\mathbf{c}$ respectively, then one can express the set of unit vectors $\left\{\boldsymbol{\sigma}_{k}\right\}$ in terms of complex vectors $Z$ and $\bar{Z}$.

$$
\boldsymbol{\sigma}_{1}=\frac{Z+\bar{Z}}{2 a}
$$




$$
\begin{gathered}
\boldsymbol{\sigma}_{2}=\frac{Z-\bar{Z}}{2 \mathbf{i} b} \\
\boldsymbol{\sigma}_{3}=\frac{Z \bar{Z}-\bar{Z} Z}{4 a b}
\end{gathered}
$$

From the scalar product $Z \cdot \bar{Z}$ the unit scalar can be expressed as

$$
1=\frac{Z \bar{Z}+\bar{Z} Z}{2\left(a^{2}+b^{2}\right)}
$$

Here, $a$ and $b$ are magnitudes of the respective vectors and the magnitude $|\mathbf{c}|=2 a b$. The algebra of complex vectors $Z$ and its conjugate $\bar{Z}$ generates complex vector space. The basis elements $\left\{1, \boldsymbol{\sigma}_{k}\right.$; $k=1,2,3\}$ form a closed complex four dimensional linear space. When the magnitudes of vectors $\mathbf{a}$ and $\mathbf{b}$ are equal, the complex vectors $Z$ and $\bar{Z}$ represent complex null vectors and these are expressed as

$$
\begin{aligned}
& Z=a\left(\boldsymbol{\sigma}_{1}+\mathbf{i} \boldsymbol{\sigma}_{2}\right), \\
& \bar{Z}=a\left(\boldsymbol{\sigma}_{1}-\mathbf{i} \boldsymbol{\sigma}_{2}\right) .
\end{aligned}
$$

The complex null vectors represent local rotation in space and hence these are found to be important entities in space. It will be shown in the later sections that the complex vectors $Z$ and $\bar{Z}$ are analogous to the creation and annihilation operators in quantum mechanics. The physical space formed by the complex null vectors is also analogous to the physical space formed by the creation and annihilation operators. Such physical space from fermion creation and annihilation operators was introduced by Baylis [13].

\subsubsection{Geoemtric Algebra of One and Two Dimensions}

The association of complex vector $Z$ with its conjugate $\bar{Z}$ generates a space in one dimension and a unit vector $\boldsymbol{\sigma}_{1}$ along a line can be defined as in Equation (32). The set of elements $\left\{1, \boldsymbol{\sigma}_{1}\right\}$ form geometric algebra $\mathcal{G}(1)$ of one dimension and the number of elements are $2^{1}$. The algebra $\mathcal{G}(1)$ does not contain any pseudoscalar. Complexifying $\mathcal{G}(1)$ generates the geometric algebra in two dimensions $\mathcal{G}(2)$.

$$
\mathcal{G}(2)=\mathcal{G}(1)+i \mathcal{G}(1)
$$

The unit vector $\boldsymbol{\sigma}_{2}$ is defined in terms of complex vectors in Equation (33) and the product $\boldsymbol{\sigma}_{1} \boldsymbol{\sigma}_{2}=i$ represents the pseudoscalar or unit bivector. The vector $\sigma_{2}$ is now defined as $\sigma_{2}=\sigma_{1} i$. The set of elements $\left\{1, \boldsymbol{\sigma}_{1}, \boldsymbol{\sigma}_{2}, i\right\}$ form geometric algebra $\mathcal{G}(2)$ of two dimensions and the number of elements are $2^{2}$. The pseudoscalar anticommutes with all vectors of the algebra, $\boldsymbol{\sigma}_{1} i=-i \boldsymbol{\sigma}_{1}$ and $\boldsymbol{\sigma}_{2} i=-i \boldsymbol{\sigma}_{2}$. A reversion operation represented by an over bar on $\boldsymbol{\sigma}_{1} \boldsymbol{\sigma}_{2}$ changes its order $\overline{\left(\boldsymbol{\sigma}_{1} \boldsymbol{\sigma}_{2}\right)}=\boldsymbol{\sigma}_{2} \boldsymbol{\sigma}_{1}$, therefore $\bar{i}=-i$. A general multivector in $\mathcal{G}(2)$ can be expressed as a combination of scalar, vector and bivector.

$$
M=\alpha+\mathbf{d}+i \delta
$$

Here, $\alpha$ and $\delta$ are scalars and $\mathbf{d}$ is a vector. The even part of this multivector is a complex scalar and odd part is a vector and the geometric algebra $\mathcal{G}(2)$ can be split into even and odd parts.

$$
\mathcal{G}(2)=\mathcal{G}^{+}(2)+\mathcal{G}^{-}(2)
$$


A multivector in $\mathcal{G}(2)$ is then split into even and odd parts represented by

$$
\begin{gathered}
M^{+}=\alpha+i \delta, \\
M^{-}=\mathbf{d} .
\end{gathered}
$$

Because of the property $i^{2}=-1$, the even multivectors in $\mathcal{G}^{+}(2)$ are complex scalars and and isomorphic to complex numbers. Then the even multivectors can be expressed in an exponential form by setting $\alpha=\rho \cos \theta$ and $\delta=\rho \sin \theta$.

$$
M^{+}=\rho(\cos \theta+i \sin \theta)=\rho e^{i \theta}
$$

This is a rotor and the pseudoscalar is visualized as the generator of rotations in the bivector plane $\boldsymbol{\sigma}_{1} \boldsymbol{\sigma}_{2}$. The product of two even multivectors gives another even multivector. Thus, $\mathcal{G}^{+}(2)$ is closed under multiplication and hence it forms subalgebra of $\mathcal{G}(2)$ and when $\rho=1$, they form a group called rotation group $S O(2)$. The unit complex numbers form a group called unitary group $U(1)$ which is isomorphic to $S O(2)$. A complex vector in $\mathcal{G}(2)$ can be defined as a combination of a vector and a bivector.

$$
C_{V}=\mathbf{d}+i \delta
$$

The square of complex vector $C_{V}^{2}=d^{2}-\delta^{2}$. When, $|\mathbf{d}|=\delta$ the complex vector is a null complex vector. The product $C_{V} \bar{C}_{V}=d^{2}+\delta^{2}+2 \delta i \mathbf{d}$. Thus the product $C_{V} \bar{C}_{V}$ is a multivector in $\mathcal{G}(2)$.

\subsubsection{Geometric Algebra of Euclidean Space}

The unit vector $\sigma_{3}$ is defined from a bivector product of complex vectors $Z$ and $\bar{Z}$ in Equation (34). The pseudoscalar in Euclidean space can be defined as $\mathbf{i}=\boldsymbol{\sigma}_{1} \boldsymbol{\sigma}_{2} \boldsymbol{\sigma}_{3}$. The geometric algebra $\mathcal{G}(3)$ can be generated from the complexification of the two dimensional geometric algebra $\mathcal{G}(2)$ [11].

$$
\mathcal{G}(3)=\mathcal{G}(2)+\mathbf{i} \mathcal{G}(2)
$$

The unit vector $\boldsymbol{\sigma}_{3}$ can also be defined as $\boldsymbol{\sigma}_{3}=\mathbf{i} \boldsymbol{\sigma}_{2} \boldsymbol{\sigma}_{1}$. The vectors $\left\{\boldsymbol{\sigma}_{k} ; k=1,2,3\right\}$ are basis unit right handed orthonormal vectors in three dimensional space. The pseudoscalar i represents a unit oriented volume. The square of a pseudoscalar is equal to minus one, $\mathbf{i}^{2}=-1$. Multiplying vectors $\boldsymbol{\sigma}_{k}$ by pseudoscalar form unit bivectors.

$$
B_{k}=\mathbf{i} \boldsymbol{\sigma}_{k}=\boldsymbol{\sigma}_{i} \boldsymbol{\sigma}_{j} \quad(i, j, k=1,2,3)
$$

Each unit bivector represents an oriented plane and the bivectors $\left\{B_{k} ; k=1,2,3\right\}$ form basis unit right handed orthonormal bivector oriented planes in the Euclidian space. The square of a unit bivector is equal to square root of minus one, $B_{k}^{2}=-1$. Then the unit imaginary or root minus one can be defined in two different ways by identifying either with pseudoscalar or unit bivector. Since the unit imaginary is associated with some physical plane, the correspondence with unit bivector is of particular importance. The product of all the basis bivectors is equal to positive unit scalar +1 .

$$
B_{1} B_{2} B_{3}=+1
$$


The set of elements $\left\{1, \boldsymbol{\sigma}_{k}, B_{k}, \mathbf{i} ; k=1,2,3\right\}$ form geometric algebra $\mathcal{G}(3)$ of Euclidean space and the number of elements are $2^{3}$. The geometric center is defined as that it consists of those elements of the algebra which commute with every element of the algebra [14]. As the pseudoscalar i commutes with all the elements of the algebra, it is called the geometric center of the algebra. However, algebraically the geometric center of $\mathcal{G}(3)$ is $(1, \mathbf{i})$ [14]. A general multivector in three dimensions can be expressed as a combination of scalar, vector, bivector and trivector parts

$$
M=\alpha+\mathbf{d}+\mathbf{i} \mathbf{g}+\mathbf{i} \delta
$$

where $\mathbf{d}$ and $\mathbf{g}$ are vectors, and $\alpha$ and $\delta$ are scalars and the last term $\mathbf{i} \delta$ is a trivector. Depending on the grade of elements, the geometric algebra can be expressed as a sum of even and odd parts.

$$
\mathcal{G}(3)=\mathcal{G}^{+}(3)+\mathbf{i} \mathcal{G}^{-}(3)
$$

Then the multivector can be written as a sum of even and odd parts $M=M^{+}+M^{-}$.

$$
\begin{aligned}
& M^{+}=\alpha+\mathbf{i} \mathbf{g} \\
& M^{-}=\mathbf{d}+\mathbf{i} \delta
\end{aligned}
$$

A product of two even multivectors gives another even multivector. Thus $\mathcal{G}^{+}(3)$ is closed under multiplication and hence it forms a subalgebra of $\mathcal{G}(3)$. In Equation (50), if we set $\alpha=\rho \cos (\theta / 2)$, and $\mathbf{i} \mathbf{g}=B \rho \sin (\theta / 2)$, with a unit bivector $B$ along the orientation of $\mathbf{i} \mathbf{g}$, the even multivector $M^{+}$can be expressed as

$$
M^{+}=\rho \exp (B \theta / 2)=\rho R
$$

The magnitude or the norm of an even multivector is

$$
\left|M^{+}\right|^{2}=M^{+} \bar{M}^{+}=\bar{M}^{+} M^{+}=\rho^{2} \bar{R} R=\rho^{2} R \bar{R}=\rho^{2} .
$$

Thus we have $\left|M^{+}\right|=\rho$ and $\bar{R} R=R \bar{R}=1$. When $\rho=1$, the magnitude of an even multivector equals one and they form a rotation group $\mathrm{SO}(3)$. A vector $\mathrm{x}$ can be rotated by an angle $\theta$ in a plane $B$ by using the following transformation.

$$
\mathbf{x}^{\prime}=R \mathbf{x} \bar{R}
$$

If we replace the rotor $R$ by the even multivector we have

$$
\mathbf{x}^{\prime}=M^{+} \mathbf{x} \bar{M}^{+}=\rho^{2} R \mathbf{x} \bar{R} .
$$

Therefore, the even multivector represents rotation followed by dilation. In particular the rotor in the plane $\boldsymbol{\sigma}_{1} \boldsymbol{\sigma}_{2}$ takes the form $R_{3}=\exp \left(-B_{3} \theta / 2\right)$. The even subalgebra $\mathcal{G}(3)$ is also known as spinor algebra, because it represents rotation followed by dilation and the geometric product of any two vectors, sum of a scalar and a bivector, in Euclidian space has an improved interpretation as a spinor. The algebra of spinors was invented by Hamilton and he called the algebra by the name quaternion algebra. A quaternion is in general known as a spinor because of its similarity with geometric product [15]. The even subalgebra is not commutative and it is isomorphic to quaternion algebra. If we represent the rotor by $R$, any vector can be rotated by the transformation $\mathbf{e}_{k}=R \boldsymbol{\sigma}_{k} \bar{R}$ such that the 
rotor satisfies the condition $R \bar{R}=1$. Then the rotor $R$ is an element of spin group which is a twofold covering group of the rotation group $S O(3)$ and the spin group has an isomorphic image, in the matrix formulation of Pauli matrices, the special unitary group $S U(2)$. The spinor algebra is very much useful for the formulation of Dirac theory and rigid body dynamics in classical mechanics. However, it can be verified that the odd part $M^{-}$is not a subalgebra of $\mathcal{G}(3)$. In geometric algebra $\mathcal{G}(3)$, a multivector can also be expressed as a sum of complex scalar and a complex vector parts $M=C_{S}+C_{V}$ and the physical interpretation of the structure is completely related to the pseudoscalar. The combination of scalar and trivector is called a complex scalar $C_{S}$ and the combination of vector and bivector is called a complex vector $C_{V}$.

$$
\begin{aligned}
& C_{S}=\alpha+\mathbf{i} \delta \\
& C_{V}=\mathbf{d}+\mathbf{i} \mathbf{g}
\end{aligned}
$$

In a complex scalar, the pseudoscalar replaces the function of unit imaginary such that $C_{S}$ has correspondence with normal complex numbers. The product of two complex scalars form another complex scalar, the algebra is closed under multiplication and forms a subalgebra of $\mathcal{G}(3)$. The geometric product of two complex vectors is a multivector. Thus the complex vector algebra is not closed and does not form subalgebra of geometric algebra $\mathcal{G}(3)$. From the complex null vector, we define a unit complex null vector in the form

$$
\begin{aligned}
D_{k} & =\frac{1}{2}\left(\boldsymbol{\sigma}_{i}+\mathbf{i} \boldsymbol{\sigma}_{j}\right), \\
\bar{D}_{k} & =\frac{1}{2}\left(\boldsymbol{\sigma}_{i}-\mathbf{i} \boldsymbol{\sigma}_{j}\right) .
\end{aligned}
$$

These unit complex null vectors satisfy the property $D_{k}^{2}=\bar{D}_{k}^{2}=0$ and the products

$$
\begin{aligned}
& D_{k} \bar{D}_{k}=\frac{1}{2}\left(1+\boldsymbol{\sigma}_{k}\right), \\
& \bar{D}_{k} D_{k}=\frac{1}{2}\left(1-\boldsymbol{\sigma}_{k}\right) .
\end{aligned}
$$

Thus these products are identified with self annihilating idempotents of the algebra. In particular, the products $D_{3} \bar{D}_{3}$ and $\bar{D}_{3} D_{3}$ correspond to the spinors $\mathcal{J}_{+}$and $\mathcal{J}_{-}$respectively and defined as

$$
\begin{aligned}
& \mathcal{J}_{+}=\frac{1}{2}\left(1+\boldsymbol{\sigma}_{3}\right), \\
& \mathcal{J}_{-}=\frac{1}{2}\left(1-\boldsymbol{\sigma}_{3}\right) .
\end{aligned}
$$

The idempotents $\mathcal{J}_{+}$and $\mathcal{J}_{-}$satisfy the following relations.

$$
\mathcal{J}_{ \pm} \mathcal{J}_{ \pm}=\mathcal{J}_{ \pm} ; \quad \mathcal{J}_{+} \mathcal{J}_{-}=\mathcal{J}_{-} \mathcal{J}_{+}=0
$$

One can easily find that these idempotents swallow the unit vector $\sigma_{3}$ when multiplied.

$$
\sigma_{3} \mathcal{J}_{ \pm}=\lambda \mathcal{J}_{ \pm}
$$

Thus the unit vector $\sigma_{3}$ acts as an operator to yield the eigen values $\lambda= \pm 1$. In conventional quantum mechanics these idempotents corresponds to the helicity states. Similarly one can easily verify the following relations.

$$
\boldsymbol{\sigma}_{1} \mathcal{J}_{ \pm}=\mathcal{J}_{\mp} \boldsymbol{\sigma}_{1}
$$




$$
\boldsymbol{\sigma}_{2} \mathcal{J}_{ \pm}=\mathcal{J}_{\mp} \boldsymbol{\sigma}_{2}
$$

Another important relations connected with idempotents is obtained by multiplying them by $B_{2}$ and $B_{1}$.

$$
\begin{aligned}
& -B_{2} \mathcal{J}_{+}=\sigma_{1} \mathcal{J}_{+}=\mathcal{J}_{-} \boldsymbol{\sigma}_{1} \quad \text { and } \quad B_{2} \mathcal{J}_{-}=\boldsymbol{\sigma}_{1} \mathcal{J}_{-}=\mathcal{J}_{+} \boldsymbol{\sigma}_{1} \\
& B_{1} \mathcal{J}_{+}=\boldsymbol{\sigma}_{2} \mathcal{J}_{+}=\mathcal{J}_{-} \boldsymbol{\sigma}_{2} \quad \text { and } \quad-B_{1} \mathcal{J}_{-}=\boldsymbol{\sigma}_{2} \mathcal{J}_{-}=\mathcal{J}_{+} \boldsymbol{\sigma}_{2}
\end{aligned}
$$

These relations are specifically used in the correspondence of spinor form of wavefunction. Geometrically one can visualize null complex vectors as points in space with zero magnitude, the scalars represent the magnitude of geometric elements. Normally the points are considered as scalars in geometric algebra [16]. However, it is more appropriate to treat scalars as the objects of magnitude as in conventional vector algebra. The vectors as usual are oriented line segments, the bivectors represent oriented planes and pseudoscalar represents an oriented unit volume in space.

\subsubsection{Generation of Higher Dimensional Space}

The four dimentional space can be generated from $\mathcal{G}(3)$ by defining $\mathbf{i}_{4}=\boldsymbol{\sigma}_{1} \boldsymbol{\sigma}_{2} \boldsymbol{\sigma}_{3} \boldsymbol{\sigma}_{4}$ and the vector $\sigma_{4}=\mathbf{i}_{4} \mathbf{i}$. The geometric algebra of four dimensional space can be expressed as

$$
\mathcal{G}(4)=\mathcal{G}(3)+\mathbf{i}_{4} \mathcal{G}(3)
$$

The set of elements $\left\{1, \boldsymbol{\sigma}_{i}, \boldsymbol{\sigma}_{i} \boldsymbol{\sigma}_{j}, \boldsymbol{\sigma}_{i} \boldsymbol{\sigma}_{j} \boldsymbol{\sigma}_{k}, \mathbf{i}_{4} ; i, j, k=1,2,3,4\right\}$ form geometric algebra $\mathcal{G}(4)$ of four space dimensions and the number of elements are $2^{4}$. The vectors of n-dimensional vector space generate geometric algebra $\mathcal{G}(n)$. The geometric algebra of higher dimensional space can be generated in a similar manner as above by defining a pseudoscalar.

$$
\mathcal{G}(n)=\mathcal{G}(n-1)+\mathbf{i}_{n} \mathcal{G}(n-1)
$$

The geometric algebra $\mathcal{G}(n)$ of $n$-dimensions contains a total number of elements $2^{n}$. A general multivector in $\mathrm{n}$-dimensional space can be expressed as

$$
M=a_{0}+a_{1}+a_{2}+a_{3}+\cdots+a_{n},
$$

where the subscript represents the grade of a vector. The geometric algebra $\mathcal{G}(n)$ can be split into even and odd parts.

$$
\mathcal{G}(n)=\mathcal{G}^{+}(n)+\mathcal{G}^{-}(n)
$$

Employing the procedure as above, the even multivectors of geoemetric algebra $\mathcal{G}(n)$ can be shown to represent rotations and dilations of $n$-dimensional vector space.

\subsubsection{Quaternion Algebra}

Since the basis bivectors of Euclidean space follow the same rules as those of unit imaginaries of quaternion algebra, a quaternion can be defined from the basis bivectors and such quaternions form four dimensional algebra with the basis $\left\{1, B_{k} ; k=1,2,3\right\}$. A quaternion in geometric algebra is an even multivector and can be expressed in the form

$$
Q=q_{0}+q_{k} B_{k}
$$


where $q_{\mu}(\mu=0,1,2,3)$ are real numbers and a summation convention on repeated indices is considered. A reversion operation on $Q$ changes the sign of bivectors.

$$
\bar{Q}=q_{0}-q_{k} B_{k}
$$

And the product

$$
\bar{Q} Q=\left(q_{0}^{2}+q_{1}^{2}+q_{2}^{2}+q_{3}^{2}\right)=\rho^{2} .
$$

If we set $q_{0}^{2}=d x_{0}^{2}=-c^{2} d t^{2}$ and $q_{k}^{2}=d x_{k}^{2}$, then the quaternion represents invariant length in spacetime.

$$
\bar{Q} Q=d x_{k}^{2}-c^{2} d t^{2}=d s^{2}=-c^{2} d \tau^{2}
$$

The proper length identified from the generalization of quaternion is in fact a two dimensional representation of Poincare group. The product of two quaternions $Q=q_{0}-q_{k} B_{k}$ and $P=p_{0}-p_{k} B_{k}$ can be expressed as

$$
\bar{Q} P=\alpha_{0}+\alpha_{k} B_{k}
$$

Thus the quaternions satisfy the multiplication rule and hence form subalgebra of geometric algebra. A quaternion can be normalized by choosing its length equal to one. Then a normalized quaternion represents spatial rotation.

$$
Q=\cos \frac{\theta}{2}+\left(a_{1} B_{1}+a_{2} B_{2}+a_{3} B_{3}\right) \sin \frac{\theta}{2}=e^{-B \theta / 2}
$$

Thus a normalized quaternion represents a rotor and satisfy the relation $Q \bar{Q}=1$. In Equation (79), $B=a_{k} B_{k}$ is a bivector. A rotation transformation of a vector $\mathbf{x}$ in the plane $B$ by angle $\alpha$ is represented by

$$
\mathbf{x}^{\prime}=Q \mathbf{x} \bar{Q}
$$

The transformation represents the counterclockwise rotation in the bivector plane $B$. The rotations in three dimensional space form the group representation $S O(3)$. In general, the same rotation in $S O(3)$ is represented by two unit quaternions $Q$ and $-Q$ because $Q \mathbf{x} \bar{Q}=(-Q) \mathbf{x}(-\bar{Q})$. Therefore the quaternions realize a double covering, i.e., 2:1 mapping of ordinary rotations. In other words the sphere of unit quaternions is a twofold covering group $S O(3)$ and hence the unit quaternions represent special unitary group $S U(2)$. A quaternion can also be expressed in a spinorial form.

$$
Q=\left(q_{0}-q_{3} B_{3}\right)-B_{2}\left(q_{2}-q_{1} B_{3}\right)
$$

The even multivectors in the brackets of above Equation (81) can be parameterized in the form of rotors.

$$
\begin{aligned}
& q_{0}-q_{3} B_{3}=\rho_{1} R_{1} \\
& q_{2}-q_{1} B_{3}=\rho_{2} R_{2}
\end{aligned}
$$

The quaternion $Q$ is now written as

$$
Q=\rho_{1} R_{1}-B_{2} \rho_{2} R_{2}
$$


This form of quaternion is called a spinor. In the Pauli two component spinning electron theory, one considers the column matrices or the spinors. The rotors $R_{1}$ and $R_{2}$ represent rotations in the $B_{3}$ plane. Without loss of generality, the rotors in Equation (84) can be replaced by a single rotor $\mathrm{R}$ in the spin plane $B_{3}$ with the condition that $\rho_{1}$ and $\rho_{2}$ are complex. This does not change the spinorial form of the quaternion.

$$
Q=\left(\rho_{1}-B_{2} \rho_{2}\right) R
$$

The above analysis gives a complimentary description of the spin wave function for fermions, like electrons and quarks, with the half angle rotor form satisfying the well known $4 \pi$ symmetry of the particles. The physical interpretation of the wave function requires normalization and can be achieved by redefining the quaternion as unitary and Equation (85) furnishes a most general two component spinor.

\subsection{Spacetime Algebra}

The complexification process in the complex space considered above, the geometric algebra $\mathcal{G}(3)$ is generated from $\mathcal{G}(2)$ and $\mathcal{G}(2)$ from $\mathcal{G}(1)$ by considering unit pseudoscalar identification with square root of minus one. The spacetime algebra of four dimensional space can be generated from the geometric algebra by considering square root of plus one with the identification of vector $\boldsymbol{\sigma}_{0}=\sqrt{+1}$ or $\boldsymbol{\sigma}_{0}^{2}=+1$. The unit vector $\sigma_{0}$ is identified with the unit vector along the future light cone. The set of othonormal right handed unit vectors $\left\{\gamma_{\mu} ; \mu=0,1,2,3\right\}$ form Minkowski spacetime.

$$
\gamma_{0}^{2}=1 \quad \text { and } \quad \gamma_{k}^{2}=\boldsymbol{\sigma}_{k} \gamma_{0} \boldsymbol{\sigma}_{k} \gamma_{0}=-1
$$

Normally, the vector $\boldsymbol{\sigma}_{0}$ is denoted by $\gamma_{0}$ in spacetime. The vectors $\gamma_{\mu}$ are in general known as covariant vectors. The contravariant vectors are expressed in the form

$$
\gamma^{0}=\gamma_{0} \quad \text { and } \quad \gamma^{k}=\gamma_{k}^{-1}=-\gamma_{k}
$$

The set of unit vectors $\gamma^{\mu}$ satisfy anticommutation relations

$$
\gamma_{\mu} \gamma_{\nu}+\gamma_{\nu} \gamma_{\mu}=2 \eta_{\mu \nu}
$$

where $\eta_{\mu \nu}$ is the flat spacetime metric with signature $(1,-1,-1,-1)$. It is well known that such anticommutation relations are satisfied by Dirac gamma matrices in relativistic quantum mechanics. Therefore the vectors $\gamma_{\mu}$ are isomorphic to Dirac gamma matrices. The unit vector $\sigma_{0}$ is identified with the unit vector along the future light cone and hence the vectors $\boldsymbol{\sigma}_{0}$ and $\gamma_{0}$ are equal. The set of basis vectors $\left\{\boldsymbol{\sigma}_{k}\right\}$ forms algebra for rest frame relative to the time like vector $\gamma_{0}$ and the vectors $\boldsymbol{\sigma}_{k}$ are interpreted as relative vectors.

$$
\boldsymbol{\sigma}_{k}=\gamma_{k} \gamma_{0}
$$

The set of relative vectors $\left\{\boldsymbol{\sigma}_{k}\right\}$ form a subalgebra of spacetime algebra. The products of these relative vectors represent spatial bivectors $\gamma_{i} \gamma_{j}$ in spacetime.

$$
B_{k}=\mathbf{i} \boldsymbol{\sigma}_{k}=\boldsymbol{\sigma}_{i} \boldsymbol{\sigma}_{j}=\gamma_{j} \gamma_{i}
$$


Thus $\boldsymbol{\sigma}_{k}$ in spacetime represent relative vector planes and $\mathbf{i} \boldsymbol{\sigma}_{k}$ are purely spatial bivector planes. The product $B_{1} B_{2} B_{3}=+1$ represents three dimensional hyperplane in spacetime. The unit pseudoscalar $I$ in spacetime is a product of vectors $\gamma_{0} \gamma_{1} \gamma_{2} \gamma_{3}$ and it is related to $\mathbf{i}$ by the equation

$$
\mathbf{i}=\boldsymbol{\sigma}_{1} \boldsymbol{\sigma}_{2} \boldsymbol{\sigma}_{3}=\gamma_{0} \gamma_{1} \gamma_{2} \gamma_{3}=I
$$

The pseudoscalar $I$ is a 4-vector and represents relative oriented volume in spacetime. The spacetime algebra is generated from $\mathcal{G}(3)$ by considering a space relative to the unit vector $\gamma_{0}$ but not by complexification.

$$
\mathcal{G}(1,3)=\mathcal{G}(3)+\gamma_{0} \mathcal{G}(3)
$$

Here, we introduced the square root of +1 to achieve the four dimensional spacetime generated from $\mathcal{G}(3)$. The set of elements $\left\{1, \gamma_{\mu}, \gamma_{\mu} \gamma_{\nu}, \gamma_{\mu} I, I\right\}$ form the geometric algebra of spacetime known as spacetime algebra developed by Hestenes [5]. The number of elements in this algebra is $2^{4}=16$. A general element in the algebra $\mathcal{G}(1,3)$ is represented by a multivector

$$
M=\alpha+d+F+g I+I \delta
$$

where $\alpha$ and $\delta$ are scalars, $d$ and $g$ are spacetime vectors and $F=\mathbf{c}+\mathbf{i} \mathbf{d}$ is a complex vector and the last term $I \delta$ is a four-vector. The spacetime vectors can be decomposed by a spacetime split. For any spacetime vector $p$ we use

$$
p=p^{\mu} \gamma_{\mu}=p^{0} \gamma_{0}-p^{k} \gamma_{k}
$$

And multiplying by $\gamma_{0}$ gives the so called spacetime split through the products

$$
\begin{gathered}
p \gamma_{0}=p^{0}-p^{k} \boldsymbol{\sigma}_{k}=p^{0}-\mathbf{p} \\
\gamma_{0} p=p^{0}+p^{k} \boldsymbol{\sigma}_{k}=p^{0}+\mathbf{p} \\
p^{2}=p \gamma_{0} \gamma_{0} p=p_{0}^{2}+\mathbf{p}^{2}
\end{gathered}
$$

The vector $p$ is said to be time-like, space-like and light-like when $p^{2}>0, p^{2}<0$ and $p^{2}=0$ respectively. In the case if the spacetime vector $p$ represents the proper momentum vector, then Equation (95) represents the spacetime split with energy $p^{0}$ and momentum $\mathbf{p}$. Using the decomposition of a spacetime vector, the scalar product of spacetime vectors $p$ and $q$ can be expressed as

$$
p . q=p \gamma_{0} \gamma_{0} q=\left(p^{0}-\mathbf{p}\right) \cdot\left(q^{0}+\mathbf{q}\right)=p^{0} \cdot q^{0}-\mathbf{p . q} .
$$

This is the required scalar product in the spacetime. The complex vector $F$ in spacetime is a combination of a relative vector and a spatial bivector and these are elements of subalgebra of spacetime algebra. Therefore, $F$ is called a complex vector in spacetime. A reversion operation on complex vector gives negative sign before both relative vector and spatial bivector.

$$
\bar{F}=-\mathbf{c}-\mathbf{i d}
$$

The vector $\gamma_{0}$ anticommutes with relative vectors and on the other hand it commutes with spatial bivectors. That is $\gamma_{0} \boldsymbol{\sigma}_{k} \gamma_{0}=-\boldsymbol{\sigma}_{k}$ and $\gamma_{0} \mathbf{i} \boldsymbol{\sigma}_{k} \gamma_{0}=\mathbf{i} \boldsymbol{\sigma}_{k}$. Multiplying $\bar{F}$ on both sides by $\gamma_{0}$ gives an 
operation known as relative reversion which is similar to the Hermitian operation in Dirac algebra and hence relative reversion is denoted by a dagger as super script. Relative reversion changes the sign of spatial bivectors but not the relative vectors.

$$
F^{\dagger}=\gamma_{0} \bar{F} \gamma_{0}=\mathbf{c}-\mathbf{i d}
$$

The relative vector and spatial bivector parts of a complex vector in spacetime are known as commuting and anticommuting parts with respect to $\gamma_{0}$ and these are expressed in the following form.

$$
\begin{gathered}
\mathbf{c}=\frac{1}{2}\left(F+F^{\dagger}\right) \\
\mathbf{i} \mathbf{d}=\frac{1}{2}\left(F-F^{\dagger}\right)
\end{gathered}
$$

Thus the complex vectors in spacetime can be decomposed by spacetime split into relative vectors and spatial bivectors and this is a $\gamma_{0}$ dependent operation. A unit complex vector $D_{k}$ in spacetime is a combination of unit relative vector and a unit spatial bivector.

$$
D_{k}=\frac{1}{2}\left(\boldsymbol{\sigma}_{i}+\mathbf{i} \boldsymbol{\sigma}_{j}\right)
$$

The operation of relative reversion on $D_{k}$ gives

$$
D_{k}^{\dagger}=\gamma_{0} \bar{D}_{k} \gamma_{0}=\frac{1}{2}\left(\boldsymbol{\sigma}_{i}-\mathbf{i} \boldsymbol{\sigma}_{j}\right)
$$

The unit bivectors $\boldsymbol{\sigma}_{i}$ and $\mathbf{i} \boldsymbol{\sigma}_{j}$ are commuting and anti commuting parts of complex vector $D_{k}$ with respect to the vector $\gamma_{0}$. The product of unit complex vector with its Hermitian conjugate gives the idempotents.

$$
\begin{aligned}
& D_{k} D_{k}^{\dagger}=\frac{1}{2}\left(1+\boldsymbol{\sigma}_{k}\right) . \\
& D_{k}^{\dagger} D_{k}=\frac{1}{2}\left(1-\boldsymbol{\sigma}_{k}\right) .
\end{aligned}
$$

These products are similar to the products in Equations (60) and (61) except for the relative vector nature of $\boldsymbol{\sigma}_{k}$. The unit trivectors are obtained by multiplying the pseudoscalr and the spacetime vectors $\gamma_{\mu}$.

$$
\begin{gathered}
d_{0}=\gamma_{0} \mathbf{i}=\gamma_{1} \gamma_{2} \gamma_{3} \quad \text { and } \quad d_{k}=\gamma_{k} \mathbf{i}=\gamma_{0} \gamma_{i} \gamma_{j} \\
d_{0}^{2}=+1 \quad \text { and } \quad d_{k}^{2}=-1
\end{gathered}
$$

The trivector $d_{0}$ is purely spatial and represents a three dimensional volume in spacetime and the trivectors $d_{k}$ may be called as relative bivectors just like relative vectors. Depending on the grade of elements, the spacetime algebra can be split into even and odd parts.

$$
\mathcal{G}(1,3)=\mathcal{G}^{+}(1,3)+\mathcal{G}^{-}(1,3)
$$

Then the multivectors in spacetime can be decomposed into even part and odd part, $M=M^{+}+M^{-}$.

$$
M^{+}=\alpha+F+I \delta
$$




$$
M^{-}=d+g I
$$

Even multivectors can be represented as a product of complex scalar and a quaternion.

$$
M^{+}=(\alpha+I \delta)\left(q_{0}-q_{k} B_{k}\right)=C_{S} Q
$$

After parameterization, an even multivector can be expressed as

$$
M^{+}=\rho^{1 / 2} e^{\mathbf{i} \varepsilon / 2} R
$$

Further, multiplying an even multivector $M^{+}$by a boost $L=e^{-\hat{\mathbf{v}} \phi / 2}$ does not change its form. Where, $\hat{\mathbf{v}}$ is the unit relative velocity vector and $\phi$ is the rapidity factor.

$$
M^{\prime+}=L M^{+}
$$

This is the representation of a spinor and along with Lorentz boost we express the even multivector as [17]

$$
\psi=\rho^{1 / 2} e^{\mathbf{i} \varepsilon / 2} L R=\rho^{1 / 2} e^{\mathbf{i} \varepsilon / 2} \Lambda,
$$

where $\Lambda=L R$ represents Lorentz boost followed by rotation or simply Lorentz rotation and the spinor $\psi$ is called the Hestenes spinor. The Lorentz rotor satisfies the relation $\Lambda \bar{\Lambda}=1$ and the set of all rotors form spin group of spacetime. The angle $\varepsilon$ is known as the Takabayasi-Hestens angle and gives a better interpretation for the passage of electron to positron.

\subsubsection{Three Dimensional Spacetime}

The space spanned by the unit vectors $\sigma_{1}$ and $\sigma_{2}$ is a flat space. Considering a unit vector $\gamma_{0}$ normal to the flat space forms a three dimensional spacetime. The idea of flat spacetime or spacetime in three dimensions was indeed introduced recently by Chappel et al. [18]. However, they considered the pseudoscalar $i=\sigma_{1} \sigma_{2}$ as a vector normal to the flat space instead of introducing a unit vector whose square root is equal to +1 . This method has some added advantage which can be examined below. The three dimensional spacetime can be generated from $\mathcal{G}(2)$ by considering a relative space with respect to the unit vector $\gamma_{0}$ along the direction of future light cone.

$$
\mathcal{G}(1,2)=\mathcal{G}(2)+\gamma_{0} \mathcal{G}(2)
$$

The set of othonormal right handed unit vectors $\left\{\gamma_{\mu} ; \mu=0,1,2\right\}$ form three dimensional spacetime. As usual we define spacetime vectors as

$$
\begin{gathered}
\gamma_{1}=\boldsymbol{\sigma}_{1} \gamma_{0} \quad ; \quad \gamma_{2}=\boldsymbol{\sigma}_{2} \gamma_{0} . \\
\gamma_{0}^{2}=1 ; \gamma_{k}^{2}=\boldsymbol{\sigma}_{k} \gamma_{0} \boldsymbol{\sigma}_{k} \gamma_{0}=-1
\end{gathered}
$$

The set of basis vectors $\left\{\boldsymbol{\sigma}_{k} ; k=1,2\right\}$ form algebra for rest frame relative to the time like vector $\gamma_{0}$ and the vectors $\sigma_{k}$ are interpreted as relative vectors. The products of these relative vectors represent spatial bivector $\gamma_{2} \gamma_{1}$.

$$
B=\boldsymbol{\sigma}_{1} \boldsymbol{\sigma}_{2}=\gamma_{2} \gamma_{1}
$$


The unit pseudoscalar $I_{3}$ is a three vector and defined as

$$
I_{3}=\gamma_{0} \gamma_{1} \gamma_{2}
$$

And the square of pseudoscalar is equal to minus one.

$$
I_{3}^{2}=\gamma_{0} \gamma_{1} \gamma_{2} \gamma_{0} \gamma_{1} \gamma_{2}=-1
$$

The pseudoscalar $I_{3}$ commutes with all elements of the three dimensional spacetime and hence it is the geometric center of the algebra. The set of elements $\left\{1, \gamma_{0}, \gamma_{k}, \gamma_{k} \gamma_{0}, B, I_{3}\right\}$ form the geometric algebra of three dimensional spacetime. The number of elements in this algebra is $2^{3}=8$. A general element in the algebra $\mathcal{G}(1,2)$ is represented by a multivector

$$
M=\alpha+d+\mathbf{g}+\beta B+I_{3} \delta
$$

where, $\alpha, \beta$ and $\delta$ are scalars, $d$ spacetime vector, $\mathbf{g}$ relative bivector, $B$ spatial unit bivector and the last term $I_{3} \delta$ is a trivector. The three dimensional spacetime can also be split into even and odd parts.

$$
\mathcal{G}(1,2)=\mathcal{G}^{+}(1,2)+\mathcal{G}^{-}(1,2)
$$

Now, the multivectors can be decomposed into even and odd parts.

$$
\begin{gathered}
M^{+}=\alpha+\mathbf{g}+\beta B \\
M^{-}=d+I_{3} \delta
\end{gathered}
$$

The odd multivectors are similar to complex numbers because of the property $I_{3}^{2}=-1$. Since $B^{2}=-1$, the complex scalar in three dimensional spacetime may also be defined as a sum of a scalar and a bivector just like in $\mathcal{G}(2)$.

$$
C_{S}=\alpha+\beta B
$$

This complex scalar represents spatial rotations in a plane and as usual unit bivector B is a generator of rotations in the planar space. A complex vector in three dimensional spacetime is defined as a sum of relative vector and a spatial bivector.

$$
C_{V}=\mathbf{g}+\beta B
$$

In this algebra, the spacetime events can be described by a complex vector written in the following form.

$$
T_{V}=\mathbf{x}+T
$$

Here, $T$ is a bivector time defined as $T=\gamma_{2} \gamma_{1} c t$. Thus time is treated as a plane in the local planar space. The geometrical concept of proper time as a plane defined by the angular momentum has been considered recently by Machicote [19]. The idea of proper time as a rotational entity or a bivector contradicts the steady flow of time. However, recent experiments use the rotating electric field in circularly polarized light as an ottosecond clock to probe atomic processes [20]. Such that proper time may be treated as plane of rotation. In the rest frame of the particle $d \tau=d t$. Taking reversion of the complex vector gives

$$
\bar{T}_{V}=-(\mathbf{x}+T) .
$$


Since, $\mathbf{x} T=-T \mathrm{x}$ one can find the product

$$
T_{V} \bar{T}_{V}=c^{2} t^{2}-x^{2}
$$

Identifying the product $T_{V} \bar{T}_{V}=c^{2} \tau^{2}$, the metric in the three dimensional spacetime can be obtained.

$$
c^{2} \tau^{2}=c^{2} t^{2}-x^{2}
$$

This is exactly the required form of the Minkowski metric and the method is quite straight forward. Taking the differential of complex vector $T_{V}, d T_{V}=d x+c d T$, the spacetime interval can be expressed as

$$
c^{2} d \tau^{2}=c^{2} d t^{2}-d x^{2}
$$

The proper velocity complex bivector in three dimensional spacetime is defined as

$$
U=\gamma(\mathbf{v}+c B)
$$

Taking a reversion operation on $U$ and finding the product $U \bar{U}$ gives

$$
u^{2}=U \bar{U}=c^{2}
$$

where $\gamma$ is the Lorenz factor and $u$ is the proper velocity vector. The product of proper velocity complex vector with mass of the particle gives the proper momentum complex vector.

$$
\begin{gathered}
P=m U=p+\frac{E}{c} B \\
p^{2}=P \bar{P}=\frac{E^{2}}{c^{2}}-p^{2}=m^{2} c^{2}
\end{gathered}
$$

The even mulitivectors in three dimensional spacetime may be expressed to represent Lorentz transformations in three dimensional spacetime. Now, we express the even multivector in the following form

$$
M^{+}=a_{0}-a_{1} \boldsymbol{\sigma}_{1}-a_{2} \boldsymbol{\sigma}_{2}-a_{3} B .
$$

Here, $a_{k}$ are scalars. To write this in an exponential form to represent both boost and rotation in three dimensional spacetime, we introduce the condition $\left(a_{0}^{2}+a_{3}^{2}\right)-\left(a_{1}^{2}+a_{2}^{2}\right)=1$ and then allow the following parameterization.

$$
\begin{aligned}
& a_{0}=\cosh (\phi / 2) \cos (\theta / 2) \\
& a_{1}=\frac{v_{1}}{|\mathbf{v}|} \sinh (\phi / 2) \cos (\theta / 2)-\frac{v_{2}}{|\mathbf{v}|} \sinh (\phi / 2) \sin (\theta / 2) \\
& a_{2}=\frac{v_{1}}{|\mathbf{v}|} \sinh (\phi / 2) \cos (\theta / 2)+\frac{v_{2}}{|\mathbf{v}|} \sinh (\phi / 2) \sin (\theta / 2) \\
& a_{3}=\cosh (\phi / 2) \sin (\theta / 2)
\end{aligned}
$$

In these equations, $\mathbf{v}=v_{1} \boldsymbol{\sigma}_{1}+v_{2} \boldsymbol{\sigma}_{2}$ is the relative velocity vector, $\phi$ the rapidity factor and $\theta$ is the angle of rotation in the spatial plane $B$. The even multivector is then expressed as

$$
M^{+}=e^{\hat{\mathbf{v}} \phi / 2} e^{-B \theta / 2}=L R=\Lambda
$$


where, $\hat{\mathbf{v}}$ is the unit vector along velocity vector $\mathbf{v}, L$ represents the boost and $R$ is the rotor in the bivector plane $B$. Thus, an even multivector $M^{+}$represents the Lorentz rotation in three dimensional spacetime. A general Lorentz transformation of a vector $\mathbf{v}$ is then expressed as

$$
\mathbf{x}^{\prime}=\Lambda \mathbf{x} \bar{\Lambda}
$$

Thus, the three dimensional spacetime gives a natural alternative to Minkowskii spacetime.

\subsubsection{Two Dimensional Spacetime}

Further reduction of space dimension gives two dimensional space time. Considering a unit vector $\gamma_{0}$ normal to the space vector $\boldsymbol{\sigma}_{1}$, the two dimensional spacetime can be generated from $\mathcal{G}(1)$ by considering a relative space with respect to the unit vector $\gamma_{0}$.

$$
\mathcal{G}(1,1)=\mathcal{G}(1)+\gamma_{0} \mathcal{G}(1)
$$

As usual we define spacetime vectors as

$$
\gamma_{1}=\boldsymbol{\sigma}_{1} \gamma_{0}, \quad \gamma_{0}^{2}=1 \quad \text { and } \quad \gamma_{1}^{2}=\boldsymbol{\sigma}_{1} \gamma_{0} \boldsymbol{\sigma}_{1} \gamma_{0}=-1
$$

The vectors $\boldsymbol{\sigma}_{1}$ is interpreted as relative vector $\boldsymbol{\sigma}_{1}=\gamma_{1} \gamma_{0}$. The set of elements $\left\{1, \gamma_{0}, \gamma_{1}, \gamma_{1} \gamma_{0}\right\}$ form the geometric algebra of two dimensional spacetime. The number of elements in this algebra is $2^{2}=4$. A general element in the algebra $\mathcal{G}(1,1)$ is represented by a multivector

$$
M=\alpha+d+\mathbf{g},
$$

where $\alpha$ is a scalar, $d$ spacetime vector and $\mathbf{g}$ relative vector. Multivectors can be decomposed into even part and odd part.

$$
M^{+}=\alpha+\mathbf{g} \text { and } M^{-}=d
$$

The odd multivector contains only one element. Multivector of even grade is a combination of a scalar and a relative vector. The spacetime events can be described by the even multivector written in the following form.

$$
T=c t+\mathbf{x}
$$

Here, $c t$ is a scalar, $\mathbf{x}=\sigma_{1} x$ and $T$ is the relative vector. One can find the product

$$
\bar{T} T=(c t-\mathbf{x})(c t+\mathbf{x})=c^{2} t^{2}-x^{2} .
$$

Identifying the product $\bar{T} T=c^{2} \tau^{2}$, the metric in the two dimensional spacetime is obtained.

$$
c^{2} \tau^{2}=c^{2} t^{2}-x^{2}
$$

Thus obtained metric is quite useful in the geometric representation of spacetime, where one space axis is considered and the other dimensions are suppressed. 


\subsubsection{Paravector Space}

The unit positive scalar in Equations (35) and (47) may geometrically be represented by a hyper surface in four dimensional space. Thus the fourth dimension time may be purely obtained from the scalar product of complex vectors or from the product of basis bivectors and can be represented by a vector $\boldsymbol{\sigma}_{0}=1$. This identification cuts the geometric algebra $\mathcal{G}(3)$ into half such that the set of elements $\left\{\boldsymbol{\sigma}_{\mu} ; \mu=0,1,2,3\right\}$ represent the four dimentional physical space [21] if its natural complex structure is recognized. The canonical element $\mathbf{i}$ squares to minus one and commutes with all other elements and its function is exactly similar to the unit imaginary and hence it may be identified with the field of complex numbers. In such a complex structure, every element can be expressed as a complex linear combination of basis elements $\left\{\boldsymbol{\sigma}_{\mu}\right\}$. The geometric algebra generated by real three dimensional Euclidean space thus operates in a complex four dimensional vector space which contains both the complex field and three dimensional space as subspace. A general element $p$ in complex structure of $\mathrm{Cl}_{3}$ is called as a paravector and equal to the sum of a scalar $p_{0}$ and a vector $\mathbf{p}$.

$$
p=p_{0}+\mathbf{p}
$$

Since the product of a complex vector with its conjugate is equal to the sum of a scalar and a vector, a paravector can also be defined from the product of a complex vector with its conjugate. The spatial reversal of a paravector $p$ reverses the sign of vector part.

$$
p *=p_{0}-\mathbf{p}
$$

The paravector $p *$ is also known as Clifford conjugate of $p$. Then the product

$$
p p *=p_{0}^{2}-\mathbf{p}^{2} \text {. }
$$

If $p_{0}$ and $\mathbf{p}$ represent the components of four momentum, the paravector $p$ then represents proper momentum. The paravector can be expressed in terms of basis elements as

$$
p=p^{\mu} \boldsymbol{\sigma}_{\mu}
$$

The paravector space provides the required representation for four dimensional spacetime only with the consideration of some additional set of rules.

\subsubsection{Generation of Higher Dimensional Complex Spacetime}

The method followed in the generation of $\mathcal{G}(3)$ can be extended in the same manner to generate higher space dimensions. Higher dimensional spacetime can be generated from spacetime by defining pseudoscalar $I_{4}$ with additional spatial vector $\gamma_{4}$.

$$
I_{4}=\gamma_{0} \gamma_{1} \gamma_{2} \gamma_{3} \gamma_{4}
$$

Then we have $\gamma_{4}=I I_{4}$ and $\gamma_{4}^{2}=-1$. The set of unit orthonormal vectors $\left\{\gamma_{\mu} ; \mu=0,1,2,3,4\right\}$ form a five dimensional spacetime. The pseudoscalar squares to plus one, $I_{4}^{2}=+1$. The geometric algebra 
$\mathcal{G}(1,4)$ with its geometric centre $I_{4}$ contains $2^{5}=32$ number of elements and can be represented by the equation

$$
\mathcal{G}(1,4)=\mathcal{G}(1,3)+I_{4} \mathcal{G}(1,3) .
$$

And in general this is expressed as

$$
\mathcal{G}(1, n+1)=\mathcal{G}(1, n)+I_{n+1} \mathcal{G}(1, n) .
$$

Thus the geometric algebra of higher dimensional space is generated from the identification of the corresponding pseudoscalar to the square root of minus one in the case of odd spatial dimensions and to the square root of plus one in the case of even spatial dimensions $(n>2)$ which gives the underlying complex nature of the algebra.

\subsubsection{Matrix Representation}

The basis vectors $\left\{\boldsymbol{\sigma}_{k} ; k=1,2,3\right\}$ in $\mathcal{G}(3)$ satisfy the following commutation rules.

$$
\left[\boldsymbol{\sigma}_{i}, \boldsymbol{\sigma}_{j}\right]=2 \delta_{i j}+2 \epsilon_{i j k} \boldsymbol{\sigma}_{k}
$$

The set of Lie bracket commutation relations in the Equation (154) is a local expression for group multiplication law. The coefficients $\epsilon_{i j k}$ are called structure coefficients of the Lie algebra and $\delta_{i j}$ are neutral elements and the factor 2 represents the $2: 1$ homomorphism between $S U(2)$ and $O(3)$ groups. It is well known fact that the basis vectors are isomorphic to Pauli matrices in general.

$$
\boldsymbol{\sigma}_{1}=\left(\begin{array}{cc}
0 & 1 \\
1 & 0
\end{array}\right) ; \quad \boldsymbol{\sigma}_{2}=\left(\begin{array}{cc}
0 & -i \\
i & 0
\end{array}\right) ; \quad \boldsymbol{\sigma}_{3}=\left(\begin{array}{cc}
1 & 0 \\
0 & -1
\end{array}\right)
$$

Thus the basis vectors play the role of spin operators and at the same time these represent the Pauli matrices without any ambiguity as normally encountered in quantum physics. The basis bivectors $\left\{B_{k} ; k=1,2,3\right\}$ in $\mathcal{G}(3)$ satisfy the commutation relations given by

$$
\left[B_{i}, B_{j}\right]=-2 \delta_{i j}+2 \epsilon_{i j k} B_{k}
$$

It is trivial to see that the basis bivectors are isomorphic to the matrices of the type

$$
B_{1}=\mathbf{i} \boldsymbol{\sigma}_{1}=\left(\begin{array}{cc}
0 & i \\
i & 0
\end{array}\right) ; \quad B_{2}=\mathbf{i} \boldsymbol{\sigma}_{2}=\left(\begin{array}{cc}
0 & 1 \\
-1 & 0
\end{array}\right) ; \quad B_{3}=\mathbf{i} \boldsymbol{\sigma}_{3}=\left(\begin{array}{cc}
i & 0 \\
0 & -i
\end{array}\right)
$$

Thus these matrices form a basis for the algebra of $S U(2)$ group. Any arbitrary complex vector in $\mathcal{G}(3)$ is then be represented in matrix form using Equations (155) and (157). The complex vector $C_{V}=\mathbf{a}+\mathbf{i} \mathbf{b}$ can be represented in the matrix form as

$$
C_{V}=\left(\begin{array}{cc}
a_{3}+i b_{3} & \left(a_{1}+b_{2}\right)-i\left(a_{2}-b_{1}\right) \\
\left(a_{1}-b_{2}\right)+i\left(a_{2}+b_{1}\right) & -\left(a_{3}+i b_{3}\right)
\end{array}\right) .
$$

If we choose the vectors $\mathbf{a}$ and $\mathbf{b}$ along $\sigma_{1}$ and $\sigma_{2}$, the above matrix representation is simplified.

$$
C_{V}=\left(\begin{array}{cc}
0 & a+b \\
a-b & 0
\end{array}\right)
$$


The unit complex vector $D_{3}=(1 / 2)\left(\boldsymbol{\sigma}_{1}+\mathbf{i} \boldsymbol{\sigma}_{2}\right)$ and its conjugate $\bar{D}_{3}$ are now expressed in the matrix form as

$$
D_{3}=\left(\begin{array}{cc}
0 & 1 \\
0 & 0
\end{array}\right) ; \quad \bar{D}_{3}=\left(\begin{array}{cc}
0 & 0 \\
1 & 0
\end{array}\right) .
$$

With this correspondence the idempotents $\mathcal{J}_{+}$and $\mathcal{J}_{-}$can be represented as a basis column spinors.

$$
\begin{aligned}
& \mathcal{J}_{+}=\frac{1}{2}\left(1+\boldsymbol{\sigma}_{3}\right)=\left(\begin{array}{l}
1 \\
0
\end{array}\right) \\
& \mathcal{J}_{-}=\frac{1}{2}\left(1-\boldsymbol{\sigma}_{3}\right)=\left(\begin{array}{l}
0 \\
1
\end{array}\right)
\end{aligned}
$$

Sometimes, it is quite useful to identify the following correspondence for idempotents. When multiplied with a unit matrix the idempotents do not change.

$$
1 . \mathcal{J}_{ \pm}=\mathcal{J}_{ \pm}
$$

The matrix form of $-B_{2}$ multiplied with the idempotents gives the following relations.

$$
\begin{aligned}
& -B_{2} \mathcal{J}_{+}=\left(\begin{array}{cc}
0 & -1 \\
1 & 0
\end{array}\right)\left(\begin{array}{l}
1 \\
0
\end{array}\right)=\left(\begin{array}{l}
0 \\
1
\end{array}\right) \\
& -B_{2} \mathcal{J}_{-}=\left(\begin{array}{cc}
0 & -1 \\
1 & 0
\end{array}\right)\left(\begin{array}{l}
0 \\
1
\end{array}\right)=-\left(\begin{array}{l}
1 \\
0
\end{array}\right)
\end{aligned}
$$

In view of the above relations, the idempotents can also be represented in the following form [8].

$$
1 \rightarrow\left(\begin{array}{l}
1 \\
0
\end{array}\right) ; \quad-B_{2} \rightarrow\left(\begin{array}{l}
0 \\
1
\end{array}\right)
$$

Since, the basis bivectors are having one to one correspondence with the unit imaginaries of the Hamilton quaternion algebra, a quaternion $Q$ can be represented by a matrix

$$
Q=\left(\begin{array}{cc}
q_{0}-i q_{3} & -q_{2}-i q_{1} \\
q_{2}-i q_{1} & q_{0}+i q_{3}
\end{array}\right) .
$$

For a unit quaternion this matrix is unitary as well as unimodular, that is the determinant of $Q$ equals one. The four components of a unit quaternion matrix are called Caley-Klein parameters and in this context the unit quaternions form the group $S U(2)$. The spinor form of the quaternion $Q=\left(\rho_{1}-B_{2} \rho_{2}\right) R$ can be expressed as a column matrix by identifying 1 and $-B_{2}$ as basis column spinors.

$$
Q=\left(\begin{array}{c}
\rho_{1} R \\
\rho_{2} R
\end{array}\right)
$$

The spacetime vectors $\left\{\gamma_{\mu}\right\}$ in $\mathcal{G}(1,3)$ satisfy the anti-commutation relations given by the Equation (88). In the Dirac-Pauli representation the spacetime vectors are represented in the matrix form as

$$
\gamma^{0}=\left(\begin{array}{cc}
1 & 0 \\
0 & -1
\end{array}\right) ; \quad \gamma^{k}=\left(\begin{array}{cc}
0 & \boldsymbol{\sigma}_{k} \\
-\boldsymbol{\sigma}_{k} & 0
\end{array}\right),
$$

where 1 is the $2 \times 2$ unit matrix. It should be noted that the matrix representation of complex vectors does not contain any geometrical interpretation and may be useful to compare with matrix algebra. 


\section{Applications of Complex Vector Algebra}

For historical reasons we shall start our applications of complex vectors with the elliptic harmonic motion. It is well known that the complex vector algebra can be incorporated into several applications in the theory of electromagnetism, particularly in the propagation of electromagnetic waves. We introduce here, the photon energy multivector and photon oscillator in complex vector space. A general view of space is that it is empty and featureless. However, it is actually filled with fluctuating electromagnetic zeropoint field. Such field causes the particle to oscillate. Because of these oscillations particles in their motion deviate from their mean path. The internal oscillatory and translation motion of particles can be expressed by complex vectors. We shall discuss the elementary particle dynamics at length in this section.

\subsection{Elliptic Harmonic Motion}

The harmonic motion can be defined by an equation

$$
\ddot{\mathbf{x}}-\omega^{2} \mathbf{x}=0 .
$$

Here, $\omega$ is the frequency of oscillation and an over dot denotes the time derivative. Let $\boldsymbol{\xi}$ be the displacement of the oscillating point at a position $\mathbf{x}$. A general solution of the above equation represents a wave of elliptic harmonic motion advancing in the direction of wave vector $\mathrm{k}$ and is giveny by

$$
\boldsymbol{\xi}=\mathbf{a} \cos (\mathbf{k} \cdot \mathbf{x}-\omega t)-\mathbf{b} \sin (\mathbf{k} \cdot \mathbf{x}-\omega t)
$$

The velocity of the displaced point at any given moment is

$$
\mathbf{u}=\dot{\boldsymbol{\xi}}=\omega[\mathbf{a} \sin (\mathbf{k} \cdot \mathbf{x}-\omega t)+\mathbf{b} \cos (\mathbf{k} \cdot \mathbf{x}-\omega t)]
$$

A complex vector is defined from the Equations (171) and (172) as

$$
C=\boldsymbol{\xi}+\mathbf{i} \mathbf{u} \omega^{-1}=(\mathbf{a}+\mathbf{i} \mathbf{b}) e^{\mathbf{i}(\mathbf{k} \cdot \mathbf{x}-\omega t)} .
$$

Thus any undamped elliptic harmonic wave may be represented by a product of a complex vector and an exponential factor. The harmonic vibrations takes place in the plane of the bivector $\mathbf{i} b$ and the wave advances in the direction of $\mathbf{k}$. Thus the complex vector in this case represents a train of plane waves of elliptic harmonic motion.

\subsection{Electromagnetic Theory}

In the electromagnetic theory, we study the propagation of electromagnetic waves in the realm of Maxwell's equations. The electromagnetic field in vacuum is determined by a set of four Maxwell's equations. In the complex vector formalism in three dimensional space, we express the electric field as a vector $\mathbf{E}$ and the magnetic field as a bivector $\mathbf{i} \mathbf{B}$ and in this notation the Maxwell's equations are expressed in the following form.

$$
\nabla \wedge \mathbf{E}=-\frac{1}{c} \frac{d \mathbf{i} \mathbf{B}}{d t} ; \quad \nabla \wedge(\mathbf{i} \mathbf{B})=0 ; \quad \nabla \cdot \mathbf{E}=0 ; \quad \nabla \cdot(\mathbf{i} \mathbf{B})=-\frac{1}{c} \frac{d \mathbf{E}}{d t}
$$


Here, the identities $\nabla \wedge(\mathbf{i} \mathbf{B})=\mathbf{i}(\nabla . \mathbf{B})$ and $\nabla .(\mathbf{i} \mathbf{B})=\mathbf{i}(\nabla \wedge \mathbf{B})$ are used and these dualities of inner and outer products can be found from Equations (9) and (10). These equations can be combined into a simple and single equation by expressing the the electromagnetic field $F$ as a complex vector.

$$
F=\frac{1}{2}(\mathbf{E}+\mathbf{i} \mathbf{B})
$$

Now, the set of Maxwell's equations are written in a single equation.

$$
\left(\frac{1}{c} \frac{d}{d t}+\nabla\right) F=0
$$

Multiplying the above equation by the operator $\left(\frac{1}{c} \frac{d}{d t}-\nabla\right)$ gives the d'Alembert equation or the wave equation of the electromagnetic field

$$
\partial^{2} F=0
$$

where the operator $\partial^{2}=\frac{1}{c^{2}}\left(\frac{d^{2}}{d t^{2}}-\nabla^{2}\right)$ is the d'Alembertian operator. The product $\bar{F} F$ is now written as

$$
\bar{F} F=\frac{1}{4}\left(\mathbf{E}^{2}+\mathbf{B}^{2}\right)+\frac{1}{2} \mathbf{i}(\mathbf{E} \wedge \mathbf{B}) .
$$

Similarly, we find

$$
F \bar{F}=\frac{1}{4}\left(\mathbf{E}^{2}+\mathbf{B}^{2}\right)-\frac{1}{2} \mathbf{i}(\mathbf{E} \wedge \mathbf{B}) .
$$

The energy density of electromagnetic field can be obtained from the scalar product

$$
2 \bar{F} \cdot F=\frac{1}{2}\left(\mathbf{E}^{2}+\mathbf{B}^{2}\right) .
$$

The energy of electromagnetic field per unit volume is then expressed as

$$
W=\frac{1}{4 \pi} \int d^{3} r(F \bar{F}+\bar{F} F) .
$$

The product $\bar{F} \wedge F$ gives a vector of the form

$$
2 \bar{F} \wedge F=\mathbf{i}(\mathbf{E} \wedge \mathbf{B}) .
$$

The energy flux per unit volume is expressed as

$$
S=\frac{c}{4 \pi} \int d^{3} r(\bar{F} F-F \bar{F})
$$

where $c$ is the magnitude of velocity of the electromagnetic wave. In the case of monochromatic waves, the electromagnetic fields are periodic functions of time. The solution of the d'Alembert equation, specifically for electric field can be expressed as

$$
\mathbf{E}=\mathbf{E}_{0} e^{\mathbf{i}(\mathbf{k} \cdot \mathbf{r}-\omega t+\alpha)}
$$

In this equation the quantity $\mathbf{E}_{0}$ is a complex vector [22] and it can be expressed as $\mathbf{E}_{0}=\mathbf{a}+\mathbf{i} \mathbf{b}$ with $\mathbf{a}$ and $\mathbf{b}$ real vectors satisfying the orthogonality condition $\mathbf{a} \cdot \mathbf{b}=0$. We choose the vector $\mathbf{a}$ along $\mathbf{x}$-axis and $\mathrm{b}$ along $\mathrm{y}$-axis such that the wave will be propagating along $\mathrm{z}$-direction and then

$$
\mathbf{E}=(\mathbf{a}+\mathbf{i} \mathbf{b})[\cos (\mathbf{k} . \mathbf{r}-\omega t+\alpha)+\mathbf{i} \sin (\mathbf{k} \cdot \mathbf{r}-\omega t+\alpha)]
$$


Comparing the vector parts, we find the components of electric field vector along $\mathrm{x}$ and $\mathrm{y}$ directions.

$$
\begin{gathered}
\mathbf{E}_{x}=\mathbf{a} \cos (\mathbf{k} \cdot \mathbf{r}-\omega t+\alpha) \\
\mathbf{E}_{y}=-\mathbf{b} \sin (\mathbf{k} \cdot \mathbf{r}-\omega t+\alpha)
\end{gathered}
$$

Thus we see that the electric field vector rotates in an elliptic plane normal to the direction of propagation of the wave and the electromagnetic wave is said to be elliptically polarized. The rotation is counterclockwise for $\mathbf{E}_{0}=\mathbf{a}+\mathbf{i} \mathbf{b}$ and clockwise for $\overline{\mathbf{E}}_{0}=\mathbf{a}-\mathbf{i} \mathbf{b}$. In the case $a^{2}=b^{2}$, the electric field vector rotates in a circular plane normal to the direction of propagation of the wave and the wave is said to be circularly polarized. The electric field vector can be expressed in terms of unit complex vector $D_{k}=\frac{1}{\sqrt{2}}\left(\boldsymbol{\sigma}_{i}+\mathbf{i} \boldsymbol{\sigma}_{j}\right)$.

$$
\mathbf{E}=a D_{k} e^{(\mathbf{k} \cdot \mathbf{r}-\omega t+\alpha)}
$$

The complex vectors $D_{k}$ then describe a general state of polarization [23]. In the case either $\mathbf{a}$ or $\mathbf{b}$ equals zero, the wave is said to be linearly or plane polarized.

In the presence of sources, electromagnetic fields interact with charges and a continuum of charge distribution is characterized by a charge density $\rho(r)$ and the velocities of the charges is characterized by a vector current density J. In the Euclidian space, the Maxwell's equations in the presence of sources can be expressed as

$$
\left(\frac{1}{c} \frac{\partial}{\partial t}+\nabla\right) 2 F=\mathbf{J}
$$

In the relativistic mechanics, the electromagnetic field is expressed as a combination of relative electric field $\mathbf{E}$ and spatial bivector magnetic field $I \mathbf{B}$.

$$
F=\frac{1}{2}(\mathbf{E}+I \mathbf{B})
$$

From Equations (101) and (102), we see that the relative electric and spatial bivector fields represent commuting and anticommuting parts of the complex electromagnetic field.

$$
\begin{aligned}
& \mathbf{E}=F+\gamma_{0} \bar{F} \gamma_{0} \\
& I \mathbf{B}=F-\gamma_{0} \bar{F} \gamma_{0}
\end{aligned}
$$

Further, we express charge density and current density as a combined quantity denoted by a source field $Q$.

$$
Q=\rho-\mathbf{J}
$$

The negative sign in the above equation is associated with the signature of spacetime. Now, the Maxwell's equations take the form

$$
\left(\frac{1}{c} \frac{\partial}{\partial t}+\nabla\right) 2 F=Q
$$

A general solution of this equation can be obtained by using Green function method [24]. Let $\phi$ and A be the scalar vector potentials of the electromagnetic field. Then we define electric and magnetic fields in terms of scalar and vector potentials as

$$
\mathbf{E}=\nabla \phi-\frac{1}{c} \frac{\partial \mathbf{A}}{\partial t}
$$




$$
I \mathbf{B}=\nabla \wedge \mathbf{A}
$$

Using the Lorentz gauge condition $\nabla . \mathbf{A}+\frac{1}{c} \frac{\partial \phi}{\partial t}=0$, one can express the electromagnetic field in terms of scalar and vector potentials as

$$
2 F=\left(\frac{1}{c} \frac{\partial}{\partial t}+\nabla\right) \phi-\left(\frac{1}{c} \frac{\partial}{\partial t}+\nabla\right) \mathbf{A} .
$$

In the absence of sources, the electromagnetic field complex vector is a function of vector potential only. For the applications of complex vector method in the electromagnetic theory, the electromagnetic complex vector may be defined without the factor $1 / 2$ in the Equation (175). However, this particular definition of $F$ in the Equation (175) is quite useful in the following subsections.

\subsubsection{Photon Energy Multivector and Angular Momentum}

The concept of photon, the energy quanta of electromagnetic radiation, was introduced by Planck in the blackbody radiation formula and by Einstein in the explanation of photoelectric effect. We know that the photon is a massless bundle of electromagnetic energy and because it imparts momentum we define the momentum of photon as the ratio between energy and velocity of photon. Belinfante established the fact that the photon spin could be regarded as the angular momentum due to circulating flow of energy and this idea was well explained by Ohanian [25]. In an infinite plane wave, the electric and magnetic field vectors are perpendicular to the propagation direction. In a finite transverse extent, the field lines are closed loops and represent circulating energy flow and imply the existence of angular momentum whose orientation is in the plane of circulation and this angular momentum is the spin angular momentum of photon. Further, as the electromagnetic waves propagate, the energy also flows along the direction of propagation. Thus the photon energy may be a combination of kinetic energy and rotational energy. The electromagnetic energy density obtained from the product $\bar{F} . F$ in Equation (180) represents the energy density of photon as it propagates and thus it may be treated as the kinetic energy density of photon.

$$
\bar{F} \cdot F=\frac{1}{4}\left(E^{2}+B^{2}\right)
$$

From the product $\bar{F} \wedge F$, one can express the energy of internal electromagnetic flux flow in the bivector plane normal to the propagation direction.

$$
-\mathbf{i} \bar{F} \wedge F=\frac{1}{2}(\mathbf{E} \wedge \mathbf{B})
$$

This energy density of the photon may be identified as the rotational energy density. The energy terms in Equations (198) and (199) combine to give the total energy density of photon.

$$
\varepsilon=\frac{1}{4}\left(E^{2}+B^{2}\right)+\frac{1}{2}(\mathbf{E} \wedge \mathbf{B})=\bar{F} \cdot F-\mathbf{i} \bar{F} \wedge F
$$

Thus, the total energy density of photon is a sum of a scalar and a bivector components. We identify the scalar part as the kinetic part which shows the flow of energy in the direction of propagation and bivector part as the rotational energy flow in the plane normal to the direction of propagation over a finite extent. Thus the total energy of a photon contains kinetic energy and rotational energy components.

$$
\mathcal{E}_{\text {kin }}=\frac{1}{4 \pi} \int d^{3} r \bar{F} \cdot F
$$




$$
\mathcal{E}_{\text {rot }}=-\mathbf{i} \frac{1}{4 \pi} \int d^{3} r \bar{F} \wedge F
$$

In general, twice the kinetic energy is treated as the electromagnetic energy per unit volume and it is the energy of photon. Since the energy of a photon is expressed as momentum times its velocity, one can define kinetic momentum of a photon from Equation (201) as

$$
\mathbf{p}_{k}=\frac{\mathcal{E}_{k i n}}{\mathbf{v}},
$$

where the velocity $\mathbf{v}=\mathbf{n} c$ and $\mathbf{n}$ is the unit vector along the direction of propagation. The internal momentum vector $\pi$ representing the rotational flux flow may be defined from the Equation (202) as

$$
\boldsymbol{\pi}=\frac{\mathcal{E}_{r o t}}{\mathbf{u}}
$$

where the velocity $\mathbf{u}$ represents the internal velocity of flux flow and satisfies the condition $\mathbf{u} . \boldsymbol{\pi}=0$ and $|\mathbf{u}|=c$. Then the total energy is expressed as an even multivector.

$$
\mathcal{E}=\mathbf{p}_{k} \cdot \mathbf{v}+\boldsymbol{\pi} \wedge \mathbf{u}
$$

This definition allows us to construct photon momentum complex vector and photon velocity complex vector in the following form.

$$
\begin{gathered}
P=\mathbf{p}_{k}+\mathbf{i} \boldsymbol{\pi} \\
U=\mathbf{v}+\mathbf{i} \mathbf{u}
\end{gathered}
$$

A reversion operation on $P$ gives $\bar{P}=\mathbf{p}_{k}-\mathbf{i} \boldsymbol{\pi}$. Since the magnitudes $\left|\mathbf{p}_{k}\right|$ and $|\boldsymbol{\pi}|$ are equal, we have $P^{2}=\bar{P}^{2}=0$. Therefore, the complex vector $P$ is a complex null vector which represents the light like nature of photon. Similarly the complex velocity vector is also a complex null vector. Now, the total energy of photon is expressed as

$$
\mathcal{E}=P \bar{U}
$$

The multivector form given in the Equation (205) can be compared to the symmetric energy momentum tensor $\theta^{\mu \nu}$ with the identification of the scalar part with $\theta^{00}$ and bivector part with $\theta^{i j}$. Thus the even multivector $\mathcal{E}$ may be called as the energy momentum multivector.

The property of even multivector is that it represents rotations in the bivector plane. Then, the photon energy even multivector represents a rotor. To see this we need to consider that the energy depends on some phase factor.

$$
\mathbf{p}_{k} \cdot \mathbf{v}=\mathcal{E}_{0} \cos \omega t
$$

The bivector part can be expressed as

$$
\boldsymbol{\pi} \wedge \mathbf{u}=\hat{\mathbf{J}} \mathcal{E}_{0} \cos \omega t
$$

where $\hat{\mathbf{J}}$ is a unit bivector along the orientation of angular momentum. The phase angle is then defined as

$$
\omega t=\tan ^{-1}\left(\frac{|\boldsymbol{\pi} \wedge \mathbf{u}|}{\mathbf{p}_{k} \cdot \mathbf{v}}\right)
$$


Then, we express the multivector energy as the product of $\mathcal{E}_{0}$ times a rotor.

$$
\mathcal{E}=\mathcal{E}_{0} e^{\hat{\mathbf{J}} \omega t}
$$

This relation shows that photon contains internal complex rotations of the electromagnetic field and these rotations are analogous to the internal complex rotations or zitterbewegung of electron. The cause of these internal rotations may be attributed to the fluctuations of the zeropoint field. In Equation (212), the internal rotation is the clockwise or right-handed rotation. A reversion operation on $\mathcal{E}$ gives counterclockwise or left handed rotation.

$$
\overline{\mathcal{E}}=\mathcal{E}_{0} e^{-\hat{\mathbf{J}} \omega t}
$$

Normally, in the symmetric conditions the kinetic and rotational energies are equal in magnitude and the phase angle equals $\pi / 4$. The frequency of internal rotation is the rate per unit energy flux flow within the photon.

$$
\Omega=-\hat{\mathbf{J}} \omega=-\frac{1}{\mathcal{E}} \frac{d \mathcal{E}}{d t}
$$

Here, the frequency of internal rotation represents the counterclockwise direction and it is a bivector quantity and the magnitude of internal frequency of rotation is $\omega$. Since the frequency of internal rotation is $\omega$, the particles involved are bosons with spin magnitude $\hbar$. However, we shall see in the following section that the internal frequency of rotation is $2 \omega$ for fermions with spin magnitude $\hbar / 2$. Thus the photon consists of an internal harmonic oscillator which represents the complex internal rotations. The internal complex rotations suggest that there exists internal complex spin structure of photon. The linear momentum density of the electromagnetic field is given by the Pointing vector $\mathbf{E} \times \mathbf{B}$ and the angular momentum is the cross product with position $\mathbf{r} \times \mathbf{E} \times \mathbf{B}$. The magnetic field vector can be expressed as curl of a vector potential $\mathbf{A}$ and the angular momentum density becomes $\mathbf{E} \times \mathbf{A}$. A close inspection shows that the total angular momentum has two components; one the spin angular momentum associated with the polarization and the other the orbital angular momentum associated with the spatial distribution [23]. The total angular momentum $J$ can be split into spin angular momentum $S$ and orbital angular momentum $L[26]$.

$$
J=\frac{1}{4 \pi} \int \mathbf{E} \times \mathbf{A} d^{3} x+\frac{1}{4 \pi} \int \mathbf{E}^{n}(\mathbf{r} \times \nabla) \mathbf{A}^{n} d^{3} x=S+L
$$

It has been shown explicitly in the reference [27] that in the case of photon propagating in an arbitrary direction, say $\mathbf{n}$, the spin angular momentum and orbital angular momentum are expressed as

$$
\begin{aligned}
& S=\operatorname{in} \hbar, \\
& L=\operatorname{im} \hbar .
\end{aligned}
$$

The unit vectors $\mathbf{n}$ and $\mathbf{m}$ satisfy the orthogonality condition $\mathbf{n} . \mathbf{m}=0$ and the vector $\mathbf{m}$ lies in the plane of the unit bivector in. The spin angular momentum causes the complex vector field $F$ to rotate in the $\mathbf{E} \wedge \mathbf{B}$ plane without changing the direction of propagation vector $\mathbf{k}$. Whereas, the orbital angular momentum causes the plane having orientation defined by the bivector $\mathbf{r} \wedge \mathbf{k}$ to rotate without changing the direction of the vector $\mathbf{k}$ and the orientation of the plane $\mathbf{E} \wedge \mathbf{B}$. 


\subsubsection{The Photon Harmonic Oscillator in Complex Vector Space}

In the absence of sources, the field complex vector F can be expressed only in terms of a vector potential. First, we consider the Fourier transform of vector potential $\mathbf{A}(\mathbf{r}, t)$.

$$
\mathbf{A}(\mathbf{r}, t)=\frac{1}{2 \pi} \int \mathcal{A}(\mathbf{k}, t) e^{-i \mathbf{k} \cdot \mathbf{r}} d^{3} k
$$

The unit imaginary in the above equation is normally replaced by a bivector $\mathbf{i} \hat{\mathbf{k}}$, where $\hat{\mathbf{k}}$ is a unit vector along $\mathbf{k}$. To find $\nabla \wedge \mathbf{A}$ we need to evaluate the term $\nabla \wedge e^{-i \mathbf{k} . \mathbf{r}}$.

$\nabla \wedge e^{-i \mathbf{k} . \mathbf{r}}=-\mathbf{i} \hat{\mathbf{k}}(\nabla \wedge \mathbf{k} \cdot \mathbf{r}) e^{-i \mathbf{k} \cdot \mathbf{r}}=-\frac{\mathbf{i} \omega}{c} e^{-i \mathbf{k} . \mathbf{r}}$.

Now, the magnetic field bivector in terms of vector potential is expressed as

$$
\mathbf{i B}=\nabla \wedge \mathbf{A}=-i \mathbf{k A}=-\mathbf{i} \omega\left(\frac{\mathbf{A}}{c}\right),
$$

where $k c=\omega$. The electric field vector in terms of vector potential is defined as

$$
\mathbf{E}=-\frac{\partial}{\partial t}\left(\frac{\mathbf{A}}{c}\right) .
$$

Then the electromagnetic field complex vector $F$ is expressed as

$$
F=-\frac{1}{2}\left[\frac{\partial}{\partial t}\left(\frac{\mathbf{A}}{c}\right)+\mathbf{i} \omega\left(\frac{\mathbf{A}}{c}\right)\right] .
$$

Now, consider the canonical position and momentum coordinates $\boldsymbol{\xi}=\left(\frac{\mathbf{A}}{c}\right)$ and $\boldsymbol{\pi}=\dot{\boldsymbol{\xi}}=\frac{\partial}{\partial t}\left(\frac{\mathbf{A}}{c}\right)$. The canonical position and momentum coordinates of this form were introduced by Kobe [28] and Cohen-Tanoudji et al. [26]. The Hamiltonian representing the energy of photon is then expressed as

$$
H=\frac{1}{2}\left(\pi^{2}+\omega^{2} \xi^{2}\right)
$$

In the above expression, the integral $\frac{1}{4 \pi} \int d^{3} r$ is supressed. However, in all the expressions in this section we implicitly consider this integral wherever required but not shown explicitly. The canonical position and momentum vectors are regarded as internal coordinates of the photon and these are analogous to the internal position and momentum coordinates of an electron oscillator [12]. To analyze these internal oscillations, consider the vectors $\mathbf{a}=\omega^{1 / 2} \boldsymbol{\xi}$ and $\mathbf{b}=\boldsymbol{\pi} / \omega^{1 / 2}$ and define the complex vector $Z$ in the following form

$$
Z=k(\mathbf{a}+\mathbf{i} \mathbf{b})
$$

where the constant $k^{2}=1 / 2 \hbar$. A reversion operation on $Z$ gives

$$
\bar{Z}=k(\mathbf{a}-\mathbf{i} \mathbf{b})
$$

When $a^{2}=b^{2}$, the internal complex rotations are circular and $\bar{Z}^{2}=Z^{2}=0$. In this special case, for example in circularly polarized light, the complex vectors are complex null vectors. However, it is not necessary that the magnitudes $a$ and $b$ be equal and in the general case the complex vectors are not complex null vectors. The photon structure is characterized by the complex vectors which represent the 
internal complex rotations in complex vector space. From the Equations (223) and (224), the products $Z \bar{Z}$ and $\bar{Z} Z$ can be obtained as

$$
\begin{aligned}
& Z \bar{Z}=k^{2}\left(a^{2}+b^{2}\right)-2 \mathbf{i} k^{2}(\mathbf{a} \wedge \mathbf{b}), \\
& \bar{Z} Z=k^{2}\left(a^{2}+b^{2}\right)+2 \mathbf{i} k^{2}(\mathbf{a} \wedge \mathbf{b}) .
\end{aligned}
$$

From these products $Z \bar{Z}$ and $\bar{Z} Z$, the scalar and bivector products can be expressed in the following form.

$$
\begin{gathered}
Z \cdot \bar{Z}=\frac{1}{2}(Z \bar{Z}+\bar{Z} Z)=k^{2}\left(a^{2}+b^{2}\right)=\frac{H}{\hbar \omega} \\
\bar{Z} \wedge Z=\frac{1}{2}(\bar{Z} Z-Z \bar{Z})=2 \mathbf{i} k^{2}(\mathbf{a} \wedge \mathbf{b})=\mathbf{i} \frac{\mathbf{E} \wedge \mathbf{B}}{\hbar \omega}=\frac{\mathbf{i}}{\hbar}(S+L)
\end{gathered}
$$

In the above equation, the term $\mathbf{E} \wedge \mathbf{B}$ is equal to the energy of rotation $\mathcal{E}_{\text {rot }}$, and the total angular momentum $J=\mathcal{E}_{\text {rot }} / \omega$. Using the relations for $S$ and $L$ given by the Equations (216) and (217), the above Equation (228) is expressed as

$$
(Z \bar{Z}-\bar{Z} Z)=2(\mathbf{n}+\mathbf{m})
$$

Introducing idempotents in the form $\mathcal{J}_{n \pm}=(1 / 2)(1 \pm \mathbf{n})$ and multiplying the above equation from right gives the relation

$$
(Z \bar{Z}-\bar{Z} Z) \mathcal{J}_{n \pm}= \pm 2 \mathcal{J}_{n \pm}
$$

The property of the idempotent is that it absorbs the unit vector $\mathbf{n}$, i.e., $\mathbf{n} \mathcal{J}_{n+}=+\mathcal{J}_{n+}$ and in the above Equation (230) we have used the orthogonality condition $\mathbf{n} \cdot \mathbf{m}=0$. The Equation (230) shows that a photon has two helicity states depending on the rotation in the spin plane. Similarly introducing the idempotent in the form $\mathcal{J}_{m \pm}=(1 / 2)(1 \pm \mathbf{m})$ and multiplying the Equation (229) from right gives

$$
(Z \bar{Z}-\bar{Z} Z) \mathcal{J}_{m \pm}= \pm 2 \mathcal{J}_{m \pm}
$$

This relation shows that the orbital angular momentum bivector has two different orientations. Further, Equations (230) and (231) show that both spin and orbital angular momentum are separately conserved and the photon spin and orbital angular momenta are separately observable. In Beth's experiment [29], with circularly polarised light, only spin was measured from the rotational motion of quarter wave plate and in most of the experiments using Laguerre-Gaussian mode laser beams [30], the orbital angular momentum was measured and not along with spin. Hence spin and orbital angular momenta are separately conserved in the case of photons. Combining Equations (230) and (231) gives

$$
(Z \bar{Z}-\bar{Z} Z)\left(\mathcal{J}_{n+}+\mathcal{J}_{m+}\right)=+2\left(\mathcal{J}_{n+}+\mathcal{J}_{m+}\right)
$$

The complex vectors $Z \bar{Z}$ and $\bar{Z} Z$ represent transverse complex vector fields and are analogous to the vector fields introduced by Cohen-Tanoudgi et al. [26] in the reciprocal space. Multiplying Equations (225) and (226) from right by the idempotent $\mathcal{J}_{n+}$, we find the following relations.

$$
Z \bar{Z} \mathcal{J}_{n+}=\left(\frac{H}{\hbar \omega}+1\right) \mathcal{J}_{n+}
$$




$$
\bar{Z} Z \mathcal{J}_{n+}=\left(\frac{H}{\hbar \omega}-1\right) \mathcal{J}_{n+}
$$

These two equations satisfy Equation (230). Since the product $Z . \bar{Z}$ is a scalar product, the Equation (227) shows that the energy of the oscillator is a linear function of $\hbar \omega$. Suppose that the energy of the oscillator is expressed as $H=H^{\prime}+\hbar \omega$, and when substituted into Equations (233) and (234) gives

$$
\begin{gathered}
Z \bar{Z} \mathcal{J}_{n+}=\left(\frac{H^{\prime}}{\hbar \omega}+2\right) \mathcal{J}_{n+}, \\
\bar{Z} Z \mathcal{J}_{n+}=\left(\frac{H^{\prime}}{\hbar \omega}\right) \mathcal{J}_{n+} .
\end{gathered}
$$

These relations again satisfy the condition given by Equation (230). Similarly, if the energy is expressed as $H=H^{\prime \prime}+2 \hbar \omega$ and substituted into Equations (233) and (234) and the resulting equations again satisfy the condition given by Equation (230). The argument can be continued and finally we arrive at a conclusion that Equations (233) and (234) can be expressed as

$$
\begin{gathered}
Z \bar{Z} \mathcal{J}_{n+}=(n+2) \mathcal{J}_{n+}, \\
\bar{Z} Z \mathcal{J}_{n+}=n \mathcal{J}_{n+},
\end{gathered}
$$

where $n=0,1,2, \cdots$ is an integer. Now, the energy of the oscillator can be obtained from Equation (227).

$$
H \mathcal{J}_{n+}=Z . \bar{Z} \hbar \omega \mathcal{J}_{n+}=(n+1) \hbar \omega \mathcal{J}_{n+}
$$

Thus the energy of the photon harmonic oscillator becomes

$$
E_{n}=(n+1) \hbar \omega .
$$

The ground state energy of the oscillator corresponds to circular complex rotations which satisfy the condition $a^{2}=b^{2}$. In this case Equations (237) and (238) can be expressed as

$$
Z \bar{Z} \mathcal{J}_{n+}=2 \mathcal{J}_{n+} \text { and } \bar{Z} Z \mathcal{J}_{n+}=0 .
$$

The ground state energy of the oscillator is then equal to

$$
E_{0}=\hbar \omega .
$$

Thus the zeropoint energy of photon is $\hbar \omega$ and the spin frequency of photon is $\omega$. However, if the zeropoint radiation field contains $N$ number of photons in a unit volume, each photon has its polarization in an arbitrary direction and to find the average energy of zeropoint radiation, one must consider a stochastic average of all the orientations in space. Such an average simply gives a factor $1 / 2$ and the zeropoint radiation per mode becomes $\hbar \omega / 2$. Higher energies of the oscillator correspond to the harmonic frequencies and the photon energy becomes $2 \hbar \omega, 3 \hbar \omega \cdots$ etc. To find more insight into the problem, let us consider a set of orthonormal unit vectors $\left\{\boldsymbol{\sigma}_{a}, \boldsymbol{\sigma}_{b}, \mathbf{n}\right\}$ along $\mathbf{a}, \mathbf{b}$ and propagation direction respectively. Then, the complex vectors $\bar{Z}$ and $Z$ are expressed as

$$
\bar{Z}=k \boldsymbol{\sigma}_{a}(a+b \mathbf{n}) \quad \text { and } \quad Z=k \boldsymbol{\sigma}_{a}(a-b \mathbf{n}) .
$$


Now, it is easy to show the following relations.

$$
\begin{aligned}
& Z \bar{Z} \mathcal{J}_{n+}=k^{2}(a+b)^{2} \mathcal{J}_{n+} \\
& \bar{Z} Z \mathcal{J}_{n+}=k^{2}(a-b)^{2} \mathcal{J}_{n+} .
\end{aligned}
$$

Comparing Equations (244) and (245) with Equations (237) and (238), we find

$$
2 k a=\sqrt{(n+2)}+\sqrt{n}, \quad 2 k b=\sqrt{(n+2)}-\sqrt{n} \quad \text { and } \quad 4 k^{2} a b=2 .
$$

Since $4 k^{2} a b=2$, the photon spin remains constant with its magnitude $a b=\hbar$. Multiplying the complex vectors in Equation (243) with the idempotent $\mathcal{J}_{n+}$ gives

$$
\bar{Z} \mathcal{J}_{n+}=\sqrt{(n+2)} \boldsymbol{\sigma}_{a} \mathcal{J}_{n+} \quad \text { and } \quad Z \mathcal{J}_{n+}=\sqrt{n} \boldsymbol{\sigma}_{a} \mathcal{J}_{n+}
$$

If we define a multivector $N=Z \bar{Z}$ analogous to the number operator, it satisfies the relation $N \mathcal{J}_{n+}=n \mathcal{J}_{n+}$ and we have

$$
\begin{gathered}
N \bar{Z} \mathcal{J}_{n+}=\bar{Z}(n+2) \mathcal{J}_{n+}, \\
N Z \mathcal{J}_{n+}=(Z \bar{Z}-2) Z \mathcal{J}_{n+}=Z(n-2) \mathcal{J}_{n+} .
\end{gathered}
$$

From the above relations we find the following commutation relations.

$$
[N, \bar{Z}] \mathcal{J}_{n+}=2 \bar{Z} \mathcal{J}_{n+} \quad \text { and } \quad[N, Z] \mathcal{J}_{n+}=-2 Z \mathcal{J}_{n+}
$$

In the case of ground state of the oscillator, the above equations reduce to

$$
N \bar{Z} \mathcal{J}_{n+}=(+2) \bar{Z} \mathcal{J}_{n+} \quad \text { and } \quad N Z \mathcal{J}_{n+}=(-2) Z \mathcal{J}_{n+} .
$$

These equations indicate the fact that when one photon is created or destroyed always two modes of zeropoint field are involved and gives further implication that when one photon is created there may be a particle pair (electron and positron) annihilated and similarly when one photon is destroyed there may be a particle pair created in the zeropoint fields. The transverse quantum fields defined by creation and annihilation operators $a^{\dagger}$ and $a$ satisfy the commutation relation of the same type given in the Equation (251) and therefore the complex vectors $\bar{Z}$ and $Z$ resemble the operators $a^{\dagger}$ and $a$ respectively. The consequence of zeropoint fluctuations leads to internal complex rotations and in turn we define the angular momentum of photon as the angular momentum of zeropoint field. In the above treatment we have analyzed the complex rotations of photon in terms of the oscillations of the harmonic oscillator in the complex vector formalism pertaining to internal spin rotations. However, the internal complex rotations are not only limited to the rotations pertaining to the plane of spin angular momentum but also exists in the plane of orbital angular momentum. Starting from Equation (231), it is trivial to show that the corresponding equations representing complex rotations of photon pertaining to the orbital angular momentum. In the Laguerre-Gaussian modes of laser beams it has been shown explicitly in the quantum mechanical approach that the orbital angular momentum of light beams resembles the angular momentum of harmonic oscillator [31]. 


\subsection{Elementary Particle Dynamics}

In the Dirac relativistic theory, electron executes rapid oscillations superimposed on its translational motion. Schrödinger examined the behavior of Dirac electron and discovered the so called zitterbewegung motion or the trembling motion of electron. In the Schrödinger's zitterbewegung derivation [32,33], the Dirac Hamiltonian for a free electron can be expressed as

$$
H=c \boldsymbol{\alpha} \cdot \mathbf{p}+m c^{2} \alpha_{0}
$$

where the set of matrices $\left\{\alpha_{\mu} ; \mu=0,1,2,3\right\}$ are known as Dirac matrices and are given by

$$
\alpha_{0}=\left(\begin{array}{cc}
1 & 0 \\
0 & -1
\end{array}\right) \quad \text { and } \quad \alpha_{k}=\left(\begin{array}{cc}
0 & \sigma_{k} \\
-\sigma_{k} & 0
\end{array}\right) \text {. }
$$

The matrices $\left\{\sigma_{k} ; k=1,2,3\right\}$ are the usual Pauli spin matrices. These matrices are related to the $\gamma$-matrices by the relations $\alpha_{0}=\gamma_{0}$ and $\alpha_{k}=\gamma_{0} \gamma_{k}$. The Dirac matrices satisfy the following anti-commutation relations.

$$
\left\{\alpha_{i}, \alpha_{j}\right\}=\alpha_{i}, \alpha_{j}+\alpha_{j}, \alpha_{i}=2 \delta_{i j} 1 ; \quad\left\{\alpha_{i}, \alpha_{0}\right\}=0 \quad \text { and } \quad \alpha_{0}^{2}=1
$$

The position $\hat{x}$ and momentum $\hat{p}$ operators of the electron satisfy the quantum commutation relation.

$$
[\hat{x}, \hat{p}]=i \hbar
$$

In the Heisenberg picture all these relations hold at any time and the time derivative of an operator $\hat{a}$ is given by

$$
\frac{d \hat{a}}{d t}=\frac{i}{\hbar}[H, \hat{a}]
$$

When we replace the operator $\hat{a}$ in the above equation with the position operator $\hat{x}$ and using the operator form of the Hamiltonian given in Equation (252), we find $\dot{x}=c \alpha$ and similarly one can find

$$
-i \hbar \dot{\alpha}=2 H\left(\alpha-c H^{-1} p\right)=2 H \eta
$$

Here, $\eta=\alpha-c H^{-1} p$. Since, the time derivative $\dot{\eta}=\dot{\alpha}$ the above equation can be expressed as

$$
\dot{\eta}=\frac{i 2 H}{\hbar} \eta
$$

The solution for this first order differential equation is

$$
\eta=\eta(0) \exp \left(\frac{i 2 H}{\hbar}\right)
$$

where $\eta(0)=\alpha(0)-c H^{-1} p$. Now, combining the above equation with $\dot{x}=c \alpha$ gives

$$
\dot{x}=c^{2} H^{-1} p+\left[c \alpha(0)-c^{2} H^{-1} p\right] \exp \left(\frac{i 2 H}{\hbar}\right) .
$$

Upon integration of this equation one finds

$$
x=(x(0)+\dot{x} t)+\frac{1}{2} i \hbar\left[c \alpha(0)-c^{2} H^{-1} p\right] H^{-1} \exp \left(\frac{i 2 H}{\hbar}\right) .
$$


The first term represents the position of the particle and the second term represents the internal oscillations of the particle. The cause of these oscillations has been attributed to the presence of electromagnetic fluctuating zeropoint field throughout space. Hence the particle charge oscillates from the mean position of the particle. The frequency of internal oscillations is $2 \omega=2 m c^{2} \hbar^{-1}$. Replacing the unit imaginary in Equation (261) by pseudoscalar the position coordinate can be expressed as a complex vector

$$
X=\mathbf{x}(t)+\mathbf{i} \boldsymbol{\xi}(t),
$$

where, $\mathbf{x}(t)$ represents the average position of the electron or the center of mass point and $\boldsymbol{\xi}(t)$ the center of oscillating charge point. This form is similar to the coordinate considered by Barut and Zanghi [34] with the exception that $\mathbf{i} \boldsymbol{\xi}$ is a bivector. Thus the structure of an elementary charged particle is not definitely a point particle with charge and mass or a spherical rigid body with charge distribution. The structure of electron is visualized as the point charge in a circular motion, the frequency of rotation is equal to the zitterbewegung frequency and the average radius of rotation is equal to half the Compton wavelength. The angular momentum of this internal rotation represents the electron spin. The circular motion is observed from the rest frame positioned at the centre of rotation which is also the centre of mass point. Thus the centre of mass point and the centre of charge point are separated by the radius of rotation. Differentiating Equation (262) with respect to time gives

$$
U=\mathbf{v}(t)+\mathbf{i} \mathbf{u}(t)
$$

where, $U=d X / d t, \mathbf{u}=d \boldsymbol{\xi} / d t$ is the internal instantaneous velocity whose magnitude is equal to the velocity of light and $\mathbf{v}$ is the velocity of the center of mass point.

The idea that the electron spin generated from the circular motion of zitterbewegung was advocated by several researchers. In particular, in the Hestenes model of Dirac electron [35-37] the spin was considered as a dynamical property of electron motion and described as circulation of electron mass and charge. In the extensions of semiclassical theories, the spin was identified with a bivector and point particle executes circular motion by absorbing energy from zero point field $[8,38]$. The angular momentum of this internal rotation represents particle spin and it has been explicitly shown as a bivector quantity representing the orientation of the plane of rotation $[39,40]$. The internal angular momentum or the particle bivector spin is expressed as

$$
S=\boldsymbol{\xi} \wedge \pi
$$

where, $\boldsymbol{\pi}=m \mathbf{u}$ is the internal momentum of the particle in its rest frame. The approach of extended particle structure was developed by Wyssenhoff and Raabe [41], Barut and Zhanghi [34], Salesi and Recami [42] and others. The mass of the particle is seen as the energy of oscillations confined to a region of space of dimensions of the order of Compton wavelength [33]. The local spin rotation produced by the zeropoint field allows us to interpret the mass of the particle as the local spatial complex rotation in the particle rest frame [12]. The local spin rotation produced by the zeropoint fields makes it to propose the local complex rotation as the mass of the particle. This local complex rotation will be treated in a rest frame with its origin at the center of rotation or equivalently the center of mass and may be called as the particle rest frame. Since the deviation in position $\boldsymbol{\xi}$ is considered orthogonal to $\mathbf{x}$, the velocity $\mathbf{u}$ of 
the particle internal motion is orthogonal to the particle translational velocity $\mathbf{v}$. A reversion operation on $U$ gives

$$
\bar{U}=\mathbf{v}-\mathbf{i} \mathbf{u},
$$

and the scalar product

$$
\bar{U} \cdot U=v^{2}+u^{2} .
$$

In the particle rest frame $\mathbf{v}=0$ and $\bar{U} \cdot U=u^{2}$. Since the particle internal velocity in the particle rest frame $u=c$ the velocity of light, $|U|=u=c$. However, when the particle is observed from an arbitrary frame different from the rest frame of the particle center of mass, as the centre of mass moves with velocity $\mathbf{v}$, the particle motion contains both translational and internal rotational motion of the particle. Then the particle internal velocity can be seen as

$$
u^{2}=c^{2}-v^{2}
$$

This can be expressed as

$$
u=c\left(1-\boldsymbol{\beta}^{2}\right)^{1 / 2}=c \gamma^{-1},
$$

where $\boldsymbol{\beta}=\mathbf{v} / c$ and the factor $\gamma$ is the usual Lorentz factor. The angular frequency of rotation of the particle internal motion is equal to the ratio between the velocity $c$ and radius of rotation .

$$
\omega_{s}=\frac{c}{\xi}
$$

When observed from an arbitrary frame, the angular frequency $\omega$ will be equal to the ratio between $u$ and $\xi$.

$$
\omega=\frac{u}{\xi}
$$

Expressing $u$ and $\xi$ as vectors the angular velocity is expressed as a bivector. Thus we have $-\mathbf{i} \boldsymbol{\omega}=\mathbf{u} / \boldsymbol{\xi}$ or $-\mathbf{i} \boldsymbol{\omega} \boldsymbol{\xi}=\mathbf{u}$, and Equation (270) is then expressed as

$$
\Omega=\Omega_{s} \gamma^{-1}
$$

where $\Omega=-\mathbf{i} \omega$ and $\Omega_{s}=-\mathbf{i} \omega_{s}$. Thus the angular frequency of rotation decreases when observed from an arbitrary frame and the decrease depends on the velocity of the center of mass. Considering the helical motion of the particle, this method of calculation for time dilation was first shown in a simple manner by Cavelleri [43] and also in the review by Bosi [44,45]. The above analysis shows that the basic reason for the relativistic effects that we observe is due to the internal complex rotation which is a consequence of fluctuating zeropoint fields. If the particle moves over a distance along the rotation path in $d t$ time, the proper time interval will be $d \tau=\gamma^{-1} d t$. Similarly the particle center of mass point moves over a proper distance $d x^{\prime}$, the distance observed in the arbitrary frame would be $d x^{\prime}=\gamma d x$. These equations are simply time dilation and length contraction relations. The relative velocity is then equal to $\gamma \mathbf{v}$ and the relativistic momentum is then expressed as $\mathbf{p}=\gamma m \mathbf{v}$ and this momentum in the rest frame of the particle is zero. Now, the momentum complex vector can be constructed as

$$
P=\mathbf{p}+\mathbf{i} \boldsymbol{\pi}
$$


From this momentum complex vector one can find the scalar product $P . \bar{P}$ as

$$
P \cdot \bar{P}=p^{2}+\pi^{2}
$$

Multiplying throughout by $c^{2}$ and noting $P \bar{P} c^{2}=E^{2}$ gives the relativistic energy relation in terms of momentum.

$$
E^{2}=p^{2} c^{2}+m^{2} c^{4}
$$

The above analysis confirms the fact that the special theory of relativity emerges from the internal complex rotations in the local space or in other words the relativistic effects are the manifestation of zeropoint fields and produce those results as that of the mathematical frame work of special relativity. However, in the presence of external electromagnetic field we normally replace the momentum by $\mathbf{p}-e \mathbf{A}$ in the minimal coupling prescription. Using $\mathbf{p} \rightarrow \mathbf{p}-e \mathbf{A}$ in Equation (274) and equating the scalar parts, the total energy of the particle can be expressed as

$$
E^{2}=p^{2} c^{2}-2 e c \mathbf{p} \cdot \mathbf{A}+e^{2} A^{2}+m^{2} c^{4},
$$

where A represents the zeropoint electromagnetic field vector potential. In the rest frame of the particle, i.e., when the velocity of centre of mass $\mathbf{v}=0$, the above expression reduces to

$$
E_{0} \sim m c^{2}+\frac{e^{2} A^{2}}{2 m c^{2}}
$$

In the above equation, higher order terms are neglected. Thus under the influence of zeropoint field, the term $\left(e^{2} A^{2}\right) /\left(2 m c^{2}\right)$ represents a correction to mass. The particle mass which arises due to local complex rotations in the zeropoint field is regarded as the so called bare mass and when the particle is observed from an arbitrary frame, the particle mass has some mass correction due to the presence of external zeropoint field.

\subsubsection{The Particle Spin in the Complex Vector Space}

Spin plays an important role in particle dynamics. When we consider complex internal rotations of the particle, we find some interesting results. The orbital angular momentum of the particle in a curved path of the center of mass point is a bivector quantity given by $L=\mathbf{x} \wedge \mathbf{p}$. Expanding the bivector product $X \wedge \bar{P}$ gives [12]

$$
X \wedge \bar{P}=\mathbf{x} \wedge \mathbf{p}+\boldsymbol{\xi} \wedge \boldsymbol{\pi}+\mathbf{i}(\boldsymbol{\xi} \wedge \mathbf{p})-\mathbf{i}(\mathbf{x} \wedge \boldsymbol{\pi}) .
$$

The first and second terms on right of the above equation are the orbital angular momentum and the bivector spin. The vector term $\mathbf{i}(\mathbf{x} \wedge \boldsymbol{\pi})$ represents a phase factor and the term $\mathbf{i}(\boldsymbol{\xi} \wedge \mathbf{p})$ represents an additional vector term. The last two terms in Equation (277) can be expressed as a complex vector

$$
S_{t}=S+\mathbf{i} S_{L}
$$

where $S_{L}=\boldsymbol{\xi} \wedge \mathbf{p}$ represents an oriented plane perpendicular to spin plane. The bivector $S_{L}$ can be expressed using the magnitude of average radius of rotation, which is equal to half the Comptopn wavelength $\hbar(2 m c)^{-1}$.

$$
S_{L}=\mathbf{i} \boldsymbol{\sigma}_{2} \gamma \boldsymbol{\beta} \frac{\hbar}{2}
$$


The scalar product $S_{t} \cdot \bar{S}_{t}$ is then expressed as

$$
S_{t} \cdot \bar{S}_{t}=S^{2}+S_{L}^{2}=-\gamma^{2} S^{2} .
$$

Considering $S_{t} \cdot \bar{S}_{t}=-S_{0}^{2}$, we have

$$
\left|S_{0}\right|=\gamma|S| \text {. }
$$

Multiplying this equation throughout by i $\sigma_{3}$ gives

$$
S_{0}=\mathbf{i} \boldsymbol{\sigma}_{3}\left|S_{0}\right|=\gamma S .
$$

This shows that as the particle moves with velocity $\mathbf{v}$, the magnitude of spin appears to increase by a Lorentz factor. A relation between particle mass and spin has been derived by the author [12] recently.

$$
m c^{2}=\lambda \Omega_{s} . S
$$

In this equation $\lambda= \pm 1$, and it shows the two fold nature of the equation and represents particle and antiparticle conjugation. From Equations (271) and (282) one can arrive at the relation

$$
\Omega . S_{0}=\Omega_{s} . S .
$$

It means that the particle mass remains unchanged even if the particle center of mass is in motion or in other words Equation (284) leads to the nonexistence of the so called relativistic mass. Thus a general point of view, that either in Newtonian or relativistic theories the particle mass $m$ is independent of a reference frame [46]. Thus the property of material particle mass is a conserved quantity. The bivector spin rate can be obtained by differentiating $S=\boldsymbol{\xi} \wedge \boldsymbol{\pi}$ with respect to time $t$ [12]

$$
\dot{S}=\frac{d S}{d t}=\mathbf{u} \wedge \boldsymbol{\pi}+\boldsymbol{\xi} \wedge \dot{\boldsymbol{\pi}}=-\Omega_{s} \times S,
$$

where $\dot{\boldsymbol{\pi}}$ is replaced by $-\mathbf{i} \boldsymbol{\omega}_{s} \boldsymbol{\pi}=\Omega_{s} \cdot \boldsymbol{\pi}$ and $\mathbf{u} \wedge \boldsymbol{\pi}=0$. The solution of this Equation (285) can be expressed as

$$
S(t)=S(0) e^{\Omega_{s} t}
$$

where $S(0)$ is a constant spin bivector at time $t=0$. The above Equation (286) shows that the internal spin rotation has $U(1)$ group symmetry in the rest frame of the particle. It is quite interesting to see that when the particle moves with velocity $\mathbf{v}$ the internal spin rotation rate of the particle remains constant which is a consequence of invariance of particle mass with velocity.

$$
\frac{d S_{0}}{d \tau}=-\Omega_{s} \times S
$$

Since $\dot{S}$ is equal to the constant internal energy of rotation, the spin is not a constant of motion. However, as the product $S \dot{S}=0$, the differential of square of spin bivector is zero and $S^{2}=S . S=$ $-\left|S^{2}\right|$ is a constant of motion. For an electron with charge $e$ and mass $m$, in the present complex vector treatment and in the rest frame of the particle, the charge rotation produces a current equal to $e \omega_{s}$ and the oriented area of rotation is $(\boldsymbol{\xi} \wedge \mathbf{u}) / \omega_{s}$. Then the intrinsic bivector magnetic moment $M_{0}$ lies in the same orientation of spin $S$,

$$
M_{0}=\frac{e}{m c} S
$$


and there appears to be an instantaneous dipole moment $\mathbf{d}=e \boldsymbol{\xi}$ associated with the particle. The total of electric and magnetic moments can be written as a complex vector.

$$
M_{V}=d+M_{0}=\frac{e}{m c}(m c \boldsymbol{\xi}+S) .
$$

The electromagnetic field in the rest frame of the particle center of mass can be expressed as a complex vector $F=\mathbf{E}+\mathbf{i}$ B. The interaction of $M_{V}$ with the electromagnetic field gives the interaction energy and can be expressed as

$$
E_{m}=M_{V} \cdot F=e \boldsymbol{\xi} \cdot \mathbf{E}+\frac{e}{m c} S . \mathbf{i} \mathbf{B} .
$$

The average of electric dipole interaction energy term over one complete internal rotation goes to zero. The last term in Equation (290) is a constant and represents magnetic dipole interaction energy term and gives the gyromagnetic ratio of electron $g=2$. From Equation (290), it is possible to express the spin precession in the magnetic field.

$$
\left(\frac{d S}{d t}\right)_{m}=\Omega_{m} \cdot S
$$

The angular frequency bivector $\Omega_{m}=(e / m c) \mathbf{i} \mathbf{B}$ is connected to the spin precession in the rest frame of the particle due to constant magnetic field. Then, in the presence of magnetic field, Equation (286) becomes

$$
S(t)=S(0) e^{\Omega_{t} t}
$$

Here, $\Omega_{t}=\Omega_{s}+\Omega_{m}$. Thus an external electromagnetic field leads to an additional phase in the internal spin rotation of the particle. In these equations the magnetic and electric field vectors are taken with respect to the rest frame. These fields do change for an arbitrary frame of reference and the spin equation changes accordingly.

\subsubsection{Particle Motion in Spacetime}

When the particle is in motion, the direction of internal velocity $\mathbf{u}$ is no more in the same direction as that in the rest frame of the particle. Let the new direction of $\mathbf{u}$ be denoted by a unit vector $\boldsymbol{\sigma}_{0}$. To study particle dynamics in spacetime, it is required to find the Lorentz rotation of the vector $\sigma_{0}$ and spinbivector $S$. Since both vectors are invariant under special rotation, in this section we find Lorentz boost of these vectors. In general, the Lorentz boost can be expressed in terms of rapidity factor $\varphi$ and a unit vector $\hat{\mathbf{v}}$ along particle velocity.

$$
L=\exp \left(\frac{-\hat{\mathbf{v}} \varphi}{2}\right) \quad \text { and } \quad \bar{L}=\exp \left(\frac{\hat{\mathbf{v}} \varphi}{2}\right)
$$

Here, $\bar{L}$ is the inverse of $L$. Then the active Lorentz rotation of vector $\sigma_{0}$ gives a unit complex vector along the direction of proper velocity complex vector [47].

$$
\hat{u}_{0}=\exp \left(\frac{-\hat{\mathbf{v}} \varphi}{2}\right) \boldsymbol{\sigma}_{0} \exp \left(\frac{\hat{\mathbf{v}} \varphi}{2}\right)=\boldsymbol{\sigma}_{0} \cosh \varphi+\left(\boldsymbol{\sigma}_{0} \wedge \hat{\mathbf{v}}\right) \sinh \varphi
$$

This is simply the active boost of $\sigma_{0}$ through the rapidity factor in the direction of particle velocity and generates a new complex unit vector $\hat{u}_{0}$. The bivector $\sigma_{0} \wedge \hat{\mathbf{v}}$ represents spacetime plane of rotation. 
The unit vector $\boldsymbol{\sigma}_{0}$ represents the fourth dimension in spacetime and it is same as the vector $\gamma_{0}$ considered in spacetime algebra. Multiplying the unit vector $\hat{u}_{0}$ with velocity of light $c$ gives the proper velocity complex vector $u_{0}$ of the particle. The proper velocity vector is then expressed as

$$
u_{0}=c \gamma\left(\boldsymbol{\sigma}_{0}+\boldsymbol{\sigma}_{0} \wedge \boldsymbol{\beta}\right)
$$

Multiplying Equation (295) from left by the unit vector $\boldsymbol{\sigma}_{0}$ gives the velocity four vector.

$$
u_{\mu}=\boldsymbol{\sigma}_{0} u_{0}=c \gamma(1+\boldsymbol{\beta})
$$

This type of split is called spacetime split described in Equation (95). The square of proper velocity vector is obtained as

$$
u_{0}^{2}=u_{0} \boldsymbol{\sigma}_{0} \boldsymbol{\sigma}_{0} u_{0}=c \gamma(1-\boldsymbol{\beta}) c \gamma(1+\boldsymbol{\beta})=c^{2} .
$$

Then the square of proper velocity $u_{0}^{2}$ represents the time-like case. However, the product $\bar{u}_{0} u_{0}$ is not a scalar but $\bar{u}_{0} u_{0}=\left(1+\beta^{2}\right) c^{2} \gamma^{2}+2 \boldsymbol{\beta} c^{2} \gamma^{2}$. From the definition of spin bivector one can easily find the product of spin bivector with the proper velocity $u_{0}$ turns out to be zero.

$$
S . u_{0}=\boldsymbol{\xi} \wedge m \mathbf{u} . u_{0}=0
$$

This condition is an important property of an elementary particle and responsible for the intrinsic magnetic moment. Multiplying Equation (295) throughout by $d t$ gives

$$
d t u_{0}=\gamma\left(c d t \boldsymbol{\sigma}_{0}+\boldsymbol{\sigma}_{0} \wedge d \mathbf{x}\right)
$$

Using the relation $d \tau \gamma=d t$, we have

$$
d \tau u_{0}=c d t \boldsymbol{\sigma}_{0}-\boldsymbol{\sigma}_{k} \wedge \boldsymbol{\sigma}_{0} d x_{k}
$$

In terms of spacetime basis vectors $\gamma_{\mu}$ defined in Equation (86), one can express the above equation as

$$
d \tau u_{0}=\gamma_{\mu} d x^{\mu}
$$

Then the square of proper length is

$$
c^{2} d \tau^{2}=\gamma_{\mu} d x^{\mu} \gamma_{\nu} d x^{\nu}=\eta_{\mu \nu} d x^{\mu} d x^{\nu}
$$

where $\gamma_{\mu} \gamma_{\nu}=\eta_{\mu \nu}$ is the flat spacetime metric. In a similar manner the proper momentum vector can be expressed as

$$
p=\gamma m c\left(\boldsymbol{\sigma}_{0}+\boldsymbol{\sigma}_{0} \wedge \boldsymbol{\beta}\right) .
$$

Multiplying Equation (303) from right by the unit vector $\boldsymbol{\sigma}_{0}$ gives four-vector momentum.

$$
p^{\mu}=p \boldsymbol{\sigma}_{0}=p^{0}-\mathbf{p}
$$

And the product $p^{\mu} p_{\mu}$ is expressed as

$$
p^{\mu} p_{\mu}=p \boldsymbol{\sigma}_{0} \boldsymbol{\sigma}_{0} p=\left(p^{0}-\mathbf{p}\right)\left(p^{0}+\mathbf{p}\right)=m^{2} c^{2} .
$$


Thus $p^{2}$ is a constant of motion. In the quantum notation, this is stated as the eigen state of matter $p^{2}$ has an eigen value $m^{2} c^{2}$.

To find the Lorentz rotation of spin bivector, first let us decompose the unit velocity vector $\hat{\mathbf{v}}$ into parallel and normal components with respect to the spin plane, $\hat{\mathbf{v}}=v_{p}+v_{n}$. The vectorer $v_{n}$ is along the direction of proper velocity $\hat{u}_{0}$ and it commutes with $S, S \hat{u}_{0}=\hat{u}_{0} S$. According to Equation (266), we discern that the unit vector $v_{p}$ is along the direction of internal velocity $\mathbf{u}, v_{p}=\hat{\mathbf{u}}=\mathbf{u} /|\mathbf{u}|$, and it anticommutes with $\mathrm{S}, S \hat{\mathbf{u}}=-\hat{\mathbf{u}} S$. This decomposition gives $L S \bar{L}=S \exp \hat{\mathbf{u}} \varphi$. Then, the Lorentz boost of spin bivetor is given by

$$
S^{\prime}=S(\gamma+\hat{\mathbf{u}} \gamma \beta)=S \gamma+S . \hat{\mathbf{u}} \gamma \beta
$$

The last term in the above equation is a vector and it is equal to a constant times radius of rotation. On physical grounds this term does not contribute to particle spin. In fact, when we take an average of Equation (306) over a complete rotation S.û vanishes. Now, without loss of generality, the proper spin bivector is then expressed as

$$
S_{0}=\gamma S
$$

Equation (298) shows that the scalar product of bivector spin and proper momentum vanishes.

$$
S_{0} \cdot p=S \cdot p=0
$$

Thus the spin bivector in spacetime is purely a spatial bivector. Then the tensor components of spin bivector can be expressed as $S^{0}=0$ and $S^{i j}=S$. The spin vector components are obviously written as $s^{0}=0$ and $s^{k}=s$. Further, with the identification of components $W_{0}=p . s$ and $W_{k}=p^{0} s_{k}$, a proper complex vector which corresponds to Pauli-Lubanski four-vector can be defined as

$$
W=\boldsymbol{\sigma}_{0} W_{0}+\boldsymbol{\sigma}_{0} \wedge W_{k}
$$

Multiplying the above equation from right by $\boldsymbol{\sigma}_{0}$ gives the Pauli-Lubanski four-vector $W_{\mu}$.

$$
W_{\mu}=W \boldsymbol{\sigma}_{0}=W_{0}-W_{k}
$$

Now, the product $W_{\mu} W^{\mu}$ is expressed as

$$
W_{\mu} W^{\mu}=W \boldsymbol{\sigma}_{0} \boldsymbol{\sigma}_{0} W=\left(W_{0}-W_{k}\right)\left(W_{0}+W_{k}\right)=-m^{2} s^{2} .
$$

From Equation (308) we have $W \cdot p=0$ and hence $p^{2}$ and $W^{2}$ are two independent constants of motion and in general known as first and second Casimir invariants.

When the particle moves with velocity $\mathbf{v}$ in a homogeneous and transverse magnetic field, the rate of spin precession is not same as that obtained in the rest frame of the particle. The constant magnetic field seen by the moving particle is obtained by taking Lorentz transofmation. The required transformation for the magnetic field bivector is expressed as

$$
L \mathbf{i} \mathbf{B} \bar{L}=\gamma\left(\mathbf{i} \mathbf{B}+\frac{\mathbf{i} \mathbf{B} \cdot \mathbf{v}}{c}\right) .
$$

In deriving the above equation the identity $(\mathbf{i} \mathbf{B}) \wedge \hat{\mathbf{v}}=\mathbf{i}(\mathbf{B} \cdot \hat{\mathbf{v}})$ is used. In the case of transverse magnetic field, B. $\hat{\mathbf{v}}=0$. The velocity of the particle in crossed electromagnetic fields satisfies the 
relation i B.v $=c \mathbf{E}$. Now, using the relations $d / d t=\gamma d / d \tau$ and $S_{0}=\gamma S$, the spin precession in the constant magnetic field can be expressed for $g=2$ as

$$
\left(\frac{d S_{0}}{d \tau}\right)_{m}=\frac{e}{m c}(\mathbf{E}+\mathbf{i} \mathbf{B}) \cdot S_{0}=\frac{e}{m c} F \cdot S_{0} .
$$

The electromagnetic field $F$ is the complex bivector in spacetime with relative vector $\mathbf{E}$ and spatial bivector i B. However, when $g \neq 2$ Equation (313) can be expressed as

$$
\left(\frac{d S_{0}}{d \tau}\right)_{m}=\frac{e}{m c}\left[F+\left(\frac{g}{2}-1\right) \mathbf{i} \mathbf{B}\right] \cdot S_{0} .
$$

This is simply the well known BMT equation obtained by Hestenes [48]. The above equation is generally used to measure the anomalous magnetic moment of the particles. Thus, when the particle moves in the external electromagnetic field, the particle experiences a very low angular frequency spin precession in its motion in addition to its internal complex rotations.

\subsection{Quantum Nature of Particles}

The results obtained in the previous sections provide an evidence that quantum behavior of particle is a consequence of the superposition of internal complex rotation on the translational motion and leads to the celebrated de Broglie hypothesis of wave nature of particles. The difference between internal angular frequency $\omega_{s}$ and the angular frequency $\omega$ observed from an arbitrary frame is related to the particle velocity. Dividing Equation (263) by $\xi$ and using Equations (270) and (271), we find this difference frequency [12]

$$
\omega_{B}=\beta \omega_{s}=\gamma \beta \omega .
$$

Then the wavelength associated with this frequency is

$$
\lambda_{B}=\frac{2 \pi c}{\gamma \beta \omega}=\frac{h}{p}
$$

where $h$ is the Planck's constant. This gives the genesis of de Broglie relation and the quantum nature of elementary particles in general. The wave nature of particles is therefore associated with the zeropoint fields. Uncertainty relations are said to be a consequence of wave nature of particles. However, the uncertainty principle is merely a consequence of more fundamental property of the particles related to the particle spin.

\subsubsection{Quantum and Classical Correspondence}

The Planck's idea of zeropoint energy was studied by Marshall [49] in terms of classical stochastic electrodynamics and found the relation between classical and quantum oscillators. This has inspired interesting modifications to classical electrodynamics and called as stochastic electrodynamics. Stochastic electrodynamics deals with the movement of charged particles in the classical electromagnetic fluctuating zeropint field. Stochastic electrodynamics was developed basically to derive quantum mechanics from classical physics [50-52]. The oscillatory nature of the particle is attributed to the random fluctuations defined by zeropoint field. The spectral density of stochastic zeropoint radiation 
is proportional to $\omega^{3}$ which leads to the Lorentz invariance and the field is identical from all inertial frames of reference. It is therefore called classical electromagnetic zeropoint field. The electromagnetic zeropoint field consists of fluctuating radiation that can be expressed as a superposition of polarised plane waves. In the zeropoint field, a free particle cannot remain at rest but due to random impulses from fluctuating zeropoint field, oscillates about its equilibrium position. In the treatment of classical stochastic electrodynamics, the explanation of uncertainty principle and many other quantum phenomena was shown by several authors. A complete review of stochastic electrodynamics which treats the vacuum fluctuations of quantum electrodynamics in the ground state as a real classical electromagnetic field, and a phenomenological stochastic approach to the fundamental aspects of quantum mechanics was given by de La Pena et al. [52,53]. In the stochastic electrodynamics, if the upper cut-off frequency to the spectrum of zeropoint field is not imposed, the energy of the oscillator would be divergent. Despite of its success in explaining several quantum phenomena, the results obtained in the stochastic electrodynamics have certain drawbacks; it neglects Lorentz force due to zeropoint magnetic field, it fails in the case of nonlinear forces, explanation of sharp spectral lines is not possible, diffraction of electrons cannot be explained and further the Schrödinger equation can be derived in particular cases only. Recently Cavalleri et al. [45] introduced stochastic electrodynamics with spin and explained several interesting phenomena for example, stability of elliptical orbits in an atom, the origin of special relativity and the explanation for diffraction of electrons. It has been shown that the drawbacks of stochastic electrodynamics can be removed with the introduction of spin into the problem. The particle has a natural cut-off frequency equal to the spin frequency which is the maximum frequency of the electron in the zitterbewegung interpretation. This eliminates the problem of divergence in stochastic electrodynamics. These recent advancements in the field of stochastic electrodynamics fully support the assumption that the stochastic electromagnetic field represents the zeropoint field.

In quantum mechanics we treat the particle as point particle without any structure or one may choose the cutoff radius which tends to zero. In the complex structure of a charged particle, we consider the centre of mass position and the centre of charge positions as separate. Denoting the centre of local complex rotations by the position vector $\mathrm{x}$ and the radius of rotation by the vector $\boldsymbol{\xi}$, a complex vector, connected with both the motion of the centre of mass point and internal complex rotations, is given by Equation (262). The complex spin bivector and momentum vector are given by Equations (264) and (272) respectively. In stochastic electrodynamics approach, considering a stationary solution at constant frequency of the charged particle oscillator in random zeropoint field, Boyer [54] showed that an average component of adiabatic action invariant $\langle J\rangle$ was found to be equal to $\hbar / 2$ which is in accordance with the adiabatic hypothesis of quantum theory. Then we consider the magnitude of spin angular momentum of the particle as $\hbar / 2$. One can choose any axis as the spin direction in the rest frame. Considering general quantization axis along z-axis, the spin orientation of bivector spin plane may be chosen along the plane $\mathrm{i} \boldsymbol{\sigma}_{3}$.

$$
S=\boldsymbol{\xi} \wedge \boldsymbol{\pi}=\mathbf{i} \boldsymbol{\sigma}_{3} \hbar / 2
$$

The quantum condition is the fundamental idea that leads to the development of quantum mechanics. The transition from classical to quantum mechanics can be achieved by replacing canonical conjugate dynamical variables by Hermitian operators and the non-commutativity of these operators gives the quantum conditions. The general quantum condition or the fundamental commutation relation between 
position and momentum linear operators $[\hat{x}, \hat{p}]=i \hbar$ is the basis of the whole quantum mechanics. Dirac has given an elegant generalisation of quantum condition from the dynamical theory of Poisson brackets in classical mechanics. The quantum Poisson bracket of any two dynamical variables is defined as [2]

$$
\mathbf{a b}-\mathbf{b a}=i k[\mathbf{a}, \mathbf{b}]_{P . B} .
$$

Here, the real constant $k$ must be a universal constant and chosen as the reduced Planck's constant $\hbar$ and the unit imaginary is introduced to make the left side of Equation (318) real. In the case of canonical coordinates and momenta, the Poisson bracket is replaced by $\delta_{i j}$. Now, we transform the dynamical variables into linear operators to define finally the quantum condition. Dirac wrote in his principles of quantum mechanics that if the linear operators $a$ and $b$ are real in general, the product $a b$ is not real. Further, $a b+b a$ is real and so is $i(a b-b a)$. This turns out to be the important difference between classical mechanics and quantum mechanics. The analogy between quantum mechanics and classical mechanics is obtained in the limiting case $\hbar \rightarrow 0$. In Dirac's opinion the quantum condition is a more fundamental concept than classical Poisson bracket. However, the reason behind assigning the position and momentum as operators is not exactly known, but the quantum condition is taken as a basic condition.

To find the classical correspondence in the case of the particle with internal structure, one can observe that the magnitude $|\boldsymbol{\xi}|$ refers to the average values of deviations in the position measurements and in the same state $|\boldsymbol{\pi}|$ refers to the average values of deviations in the momentum measurement. Theoretically, $|\boldsymbol{\xi}|$ gives the limit with which the position of a particle is predicted. Experimentally, $|\boldsymbol{\xi}|$ describes the accuracy with which the position of the particle is controlled. The internal position and momentum may be assumed to be equivalent to the deviations of the particle in its path and therefore we express

$$
\begin{aligned}
\boldsymbol{\xi} & =\Delta \mathbf{x}=\mathbf{n}(x-\bar{x}), \\
\boldsymbol{\pi} & =\Delta \mathbf{p}=\mathbf{m}(p-\bar{p}),
\end{aligned}
$$

where, $\bar{x}$ and $\bar{p}$ are average values of position and momentum and $\mathbf{n}$ and $\mathbf{m}$ are unit vectors along $\boldsymbol{\xi}$ and $\pi$ vectors respectively. Now, the bivector product

$$
\boldsymbol{\xi} \wedge \boldsymbol{\pi}=\mathbf{n}(x-\bar{x}) \wedge \mathbf{m}(p-\bar{p})=\mathbf{x} \wedge \mathbf{p}-\mathbf{n} \wedge \mathbf{m}(x \bar{p}-\bar{x} p)+\mathbf{n} \wedge \mathbf{m}(\bar{x} \bar{p}),
$$

where $(\mathbf{n} \wedge \mathbf{m}) x p$ is written as $\mathbf{x} \wedge \mathbf{p}$. Taking the stochastic averages of this expression on both sides gives

$$
\langle\mathbf{x} \wedge \mathbf{p}\rangle=\langle\boldsymbol{\xi} \wedge \boldsymbol{\pi}\rangle .
$$

Here, the stochastic averages $\langle\bar{x} p\rangle,\langle x \bar{p}\rangle$ and $\langle\bar{x} \bar{p}\rangle$ vanish. Without loss of generality, one can choose the unit bivector $\mathbf{n} \wedge \mathbf{m}$ equal to i $\sigma_{3}$. Using Equation (317) and expanding the bivector product $\mathbf{x} \wedge \mathbf{p}$ in the above equation gives

$$
\langle\mathbf{x p}-\mathbf{p x}\rangle=\mathrm{i} \sigma_{3} \hbar .
$$

Using the correspondence principle in quantum mechanics, replacing the vectors $\mathbf{x}$ and $\mathbf{p}$ by corresponding operators and the bivector $\mathbf{i} \sigma_{3}$ by ordinary unit imaginary, one can arrive at the quantum condition from the above equation. Thus the quantum condition is in fact connected with the particle spin. Therefore, the operators correspond to the internal structure of the particle and their postulated introduction in quantum mechanics occurs without the awareness of their existence. Then, we conclude that the classical mechanics combined with zeropoint field leads to quantum mechanics. 


\subsubsection{The Schrödinger Equation in Complex Vector Space}

The Schrödinger equation was originally derived from the modification of classical Hamilton-Jacobi equation [55]. Wigner [56] showed the connection between classical Liouville equation for a probability distribution in phase space and the Schrödinger equation for quadratic potentials. Considering the phase space evolution of an ensemble of particles described by the Wigner probability density distribution function $W(\mathbf{x}, \mathbf{p}, t)$ in phase space, the Liouville equation is expressed as

$$
\left(\frac{\partial}{\partial t}+\dot{\mathbf{x}} \frac{\partial}{\partial \mathbf{x}}+\dot{\mathbf{p}} \frac{\partial}{\partial \mathbf{p}}\right) W(\mathbf{x}, \mathbf{p}, t)=0
$$

where $\dot{\mathbf{x}}$ and $\dot{\mathbf{p}}$ are obtained from the classical Hamiltonian equations. In the presence of zeropoint vacuum fields, Dechoum et al. [57] derived the Liouvillian form of time evolution of the ensemble of particles. The classical probability amplitude is related to the Wigner distribution function. To introduce the complex nature of the wave function, one can consider a Fourier transform of the function $W(\mathbf{x}, \mathbf{p}, t)$ and using this transform in Equation (324) Dechoum et al. arrived at the Schrödinger equation. Similarly, they also derived the Pauli- Schrödinger equation from Liouville equation and it has been shown that the kinetic energy operator $-\left(\hbar^{2} / 2 m\right)\left(\partial^{2} / \partial x^{2}\right)$ has its origin in the convective operator $\dot{\mathbf{x}}(\partial / \partial \mathbf{x})$ of Equation (324) [58]. In this correspondence, we are implicitly considering the fluctuations of position coordinate and through the kinetic energy operator we incorporate zeropoint field into the Schrödinger equation [59]. In a systematic development of stochastic quantum mechanics, considering a generalized Fokker-Planck diffusion equation, de la Peña et al. [53] derived stationary state Schrödinger equation and proved that the quantum behaviour is in general a manifestation of zeropoint fields present throughout space. In the non-Markovian stochastic process, Cavelleri et al. [60,61] extended the density gradient expansion and derived a complete Schröinger equation by assuming the zitterbewegung motion of the particle in the absence of external forces. Thus the stochastic electrodynamics with spin gives a more refined Schrödinger equation in the point particle limit. Hestenes [62] pointed out that the appearance of $i \hbar$ in the Schrödinger equation is related to particle spin. Then the geometrical meaning of unit imaginary can be elucidated by identifying it as a unit bivector in the spin plane. The basic aim of these efforts is to evolve a physical theory that completely solves the problems of micro and macro aspects of matter.

To find Schrödinger equation in complex vector approach, first consider a free particle of mass $m$ and its position defined by the complex vector $X=\mathrm{x}+\mathrm{i} \boldsymbol{\xi}$, and express the partial time derivative in the following form.

$$
\frac{\partial}{\partial t}=\frac{\partial X}{\partial t} \frac{\partial}{\partial X}=\dot{X} \frac{\partial}{\partial X}
$$

Using $X=\mathbf{x}+\mathbf{i} \boldsymbol{\xi}$, expanding the partial derivative $\partial / \partial X$ gives

$$
\frac{\partial}{\partial X}=\frac{\partial}{\partial \mathbf{x}}+\mathbf{i} \boldsymbol{\xi} \frac{\partial^{2}}{\partial x^{2}}-O\left(\xi^{2}\right)
$$

Differentiating the complex vector $X$ with respect to time gives the velocity complex vector $\dot{X}=\mathbf{v}+\mathbf{i}$ u. Neglecting higher order terms in Equation (326) and substituting into Equation (325) we find

$$
\frac{\partial}{\partial t}=\mathbf{v} \frac{\partial}{\partial \mathbf{x}}+\mathbf{i} \mathbf{u} \wedge \frac{\partial}{\partial \mathbf{x}}+\mathbf{v} \wedge \mathbf{i} \boldsymbol{\xi} \frac{\partial^{2}}{\partial x^{2}}-\mathbf{u} \wedge \boldsymbol{\xi} \frac{\partial^{2}}{\partial x^{2}}
$$


The plane wave form of the wave function representing the state of the particle is defined by

$$
\psi(\mathbf{x}, t)=\rho \exp [(E t-\mathbf{p} \cdot \mathbf{x}) / 2 S]
$$

where $2 S=\mathbf{i} \boldsymbol{\sigma}_{3} \hbar$. Now, multiplying Equation (327) by the wave function $\psi(\mathbf{x}, t)$ from right gives

$$
\frac{\partial \psi}{\partial t}=\mathbf{v} \frac{\partial \psi}{\partial \mathbf{x}}+\mathbf{i} \mathbf{u} \wedge \frac{\partial \psi}{\partial \mathbf{x}}+\mathbf{v} \wedge \mathbf{i} \boldsymbol{\xi} \frac{\partial^{2} \psi}{\partial x^{2}}-\mathbf{u} \wedge \boldsymbol{\xi} \frac{\partial^{2} \psi}{\partial x^{2}}
$$

The partial derivative $\partial / \partial \mathbf{x}$ acting on the wave function gives the momentum vector. Then the first term on the right hand side of Equation (329) is a scalar. The partial derivative $\partial / \partial t$ acting on the wave function gives the spin bivector in the denominator and the second and third terms on right of Equation (329) are vector quantities. Therefore, equating the bivector quantities of Equation (329) yields

$$
\frac{\partial \psi(\mathbf{x}, t)}{\partial t}=\frac{\boldsymbol{\xi} \wedge \boldsymbol{\pi}}{m} \frac{\partial^{2} \psi(\mathbf{x}, t)}{\partial x^{2}} .
$$

Here, $S=\boldsymbol{\xi} \wedge \boldsymbol{\pi}$ is the bivector spin of the particle. Multiplying on both sides by $2 S$ and adding a potential function on right of the above equation finally gives the required Schrödinger equation.

$$
2 S \frac{\partial \psi(\mathbf{x}, t)}{\partial t}=\left[-\frac{\hbar^{2}}{2 m} \frac{\partial^{2}}{\partial x^{2}}+V(\mathbf{x})\right] \psi(\mathbf{x}, t)
$$

Thus the internal structure and the particle spin plays an important role in the foundations of quantum mechanics. The above Equation (331) gives the result that the very appearance of Planck's constant in Schrödinger theory is directly related to the existence of spin. Interestingly in discussing the macro to micro transition, Schrödinger [55] arrives at a finite spread to the particle represented by a group of waves.

\subsubsection{The Dirac Equation in Complex Vector Spacetime}

The Dirac equation in quantum mechanics is a relativistic extension of Schrödinger equation. The internal structure is hidden in both Schrödinger and the Dirac theories. The particle structure in the local rest frame is because of the local internal rotations. It has been shown that the Lorenz boost of such rotations gives the Dirac equation in complex vector spacetime [12]. In the rest frame of the particle the rotation in spin plane can be expressed by a rotor. The frequency of rotation is the frequency of spin rotation. The spin rotations are represented by half angle rotors of the form

$$
R=\exp \left(\frac{\Omega_{s}}{2} t\right)
$$

where $\Omega_{s}=-\mathbf{i} \omega$. In the present context Equation (332) can also be expressed in the following form.

$$
R=\exp \left(\frac{\Omega_{s} \cdot S}{2 S} t\right)
$$

Then the rotor equation in the rest frame can be obtained by differentiating above equation with respect to time.

$$
\dot{R}=R \frac{1}{2 S} \Omega_{s} . S
$$


The over dot on $\mathrm{R}$ represents differentiation with respect to time. Since rotor satisfies the condition $R \bar{R}=1$. Using Equation (283), the rotor equation in the rest frame of the particle is now expressed as

$$
\dot{R} 2 S=\Omega_{s} \cdot S R=\lambda m c^{2} R
$$

The value $\lambda= \pm 1$ gives the rotor equation for particle and antiparticle respectively.

$$
\begin{aligned}
& \dot{R} 2 S=+m c^{2} R \\
& \dot{\bar{R}} 2 S=-m c^{2} \bar{R}
\end{aligned}
$$

If the differential is expressed in the notation $\partial_{t}=\partial / \partial c t$, then Equation (336) can be rewritten as

$$
\partial_{t} R 2 S-m c R=0
$$

This is the form of Dirac equation in the rest frame of the particle. Multiplying with $\sigma_{0}$ from left gives

$$
\boldsymbol{\sigma}_{0} \partial_{t} R 2 S-\boldsymbol{\sigma}_{0} m c R=0
$$

When the particle is in motion as observed by an orbitrary observer, the equation of motion of the particle is obtained by applying Lorenz boost to Equation (339).

$$
L \boldsymbol{\sigma}_{0} \partial_{t} \bar{L} L R 2 S-L \boldsymbol{\sigma}_{0} m c R=0 .
$$

Using Equation (301), the term $L \boldsymbol{\sigma}_{0} \partial_{t} \bar{L}$ can be expressed as

$$
L \boldsymbol{\sigma}_{0} \partial_{t} \bar{L}=\frac{\partial}{u_{0} c \partial \tau}=\gamma_{\mu} \frac{\partial}{\partial x^{\mu}}=\partial
$$

Since, $\gamma_{0}$ is invariant under spatial rotation, Equation (340) can be written as

$$
\partial L R 2 S-m c L R \gamma_{0}=0
$$

A homogeneous Lorentz transformation is obtained by Lorentz boost followed by rotation. Then the Lorentz rotation is the product $\Lambda=L R$ and satisfies the condition $\bar{\Lambda} \Lambda=1$. Now, the equation of motion of the particle can be expressed in the following form.

$$
\partial \Lambda 2 S-m c \Lambda \gamma_{0}=0
$$

In Equation (115), we have expressed an even multivector in complex spacetime by a general spinor

$$
\psi=\rho^{1 / 2} e^{\mathbf{i} \varepsilon / 2} \Lambda
$$

where $\rho$ is the probability density and it can be defined as $\psi \bar{\psi}=\rho$ and the phase factor $e^{\mathrm{i} \varepsilon}$ is equal to +1 for $\varepsilon=0$ and -1 for $\varepsilon=\pi$. Multiplying Equation (343) by the factor $\rho^{1 / 2} e^{\mathbf{i} \varepsilon / 2}$ gives the equation

$$
\partial \psi 2 S-m c \psi \gamma_{0}=0 .
$$

This is the form of Dirac equation for spin half particles. Dirac equation for spin half antiparticles can be obtained from Equation (337) in a similar manner.

$$
\partial \psi 2 S+m c \psi \gamma_{0}=0
$$


In the presence of external electromagnetic fields, using minimal coupling prescription, we replace the momentum by $\mathbf{p}-\frac{q}{c} \mathbf{A}$, where $q$ is the particle charge. Then Equation (345) becomes

$$
\partial \psi 2 S-q \mathbf{A} \psi-m c \psi \gamma_{0}=0
$$

For an electron $q=-e$ and Equation (347) is the well known Dirac-Hestenes equation in spacetime algebra. The geometrical interpretation of Equation (347) has been extensively discussed by Hestens in several of his articles [17,35-37] and also by Boudet [63]. The Dirac theory is regarded as a fundamental theory and also a general theory. Since the wave function in Equation (345) is an even multivector, the product $\psi \bar{\psi}$ is equal to its own inverse. Since the wave function is a solution of Dirac equation, it is a spinor field. The wave function determines at every spacetime point a unique time like vector field and in the Dirac theory of electron, it is the proper current density which is defined as

$$
j=\psi c \gamma_{0} \bar{\psi}
$$

Since the pseudoscalar i anticommutes with $\gamma_{0}$, the proper current density becomes

$$
j=\rho c \Lambda \gamma_{0} \bar{\Lambda}=\rho u \text {. }
$$

Thus the above equation represents the Lorentz rotation of the vector $\gamma_{0}$ into the direction of proper current. From the Dirac equation, it follows that $\partial \rho u=0$ and hence the Dirac current is interpreted as the probability current and $\rho$ as the probability density in the local rest frame. Since the relative vector $\boldsymbol{\sigma}_{3}=\gamma_{3} \gamma_{0}$, the Dirac-Hestenes equation is invariant if we make simultaneous replacements

$$
\psi^{\prime}=\psi e^{\left(\mathrm{i} \sigma_{3} \chi\right)} \quad \text { and } \quad A^{\prime}=A-\partial \chi .
$$

This gauge invariance gives the observables of the particle motion. In this symmetry the observed proper current density is the Dirac current density.

$$
j=\psi^{\prime} c \gamma_{0} \bar{\psi}^{\prime}
$$

With the use of Equation (349), it can be shown that the Dirac current is conserved even in the presence of electromagnetic field. The spin vector is given by $\mathbf{s}=(\hbar / 2) \boldsymbol{\sigma}_{3}$ and the corresponding current is

$$
\frac{\hbar}{2} \psi c \boldsymbol{\sigma}_{3} \bar{\psi}=\frac{\hbar}{2} \psi c \gamma_{3} \gamma_{0} \bar{\psi}=\rho s u \text {. }
$$

Similarly, one can express the bivector spin current as

$$
\frac{\hbar}{2} \psi c \mathbf{i} \boldsymbol{\sigma}_{3} \bar{\psi}=\frac{\hbar}{2} \psi c \gamma_{2} \gamma_{1} \bar{\psi}=\rho c S .
$$

The plane wave solution of Dirac equation for a free particle can be expressed by decomposing the spinor $\Lambda$ into $\Lambda_{0} e^{\mathbf{p} \cdot \mathbf{x} / 2 S}$.

$$
\psi=\rho^{1 / 2} e^{\mathbf{i} \varepsilon / 2} \Lambda_{0} e^{\mathbf{p} \cdot \mathbf{x} / 2 S}
$$

Substituting this wave function in the Dirac equation for a free particle, Equation (345) gives

$$
p \psi-m c \psi \gamma_{0}=0
$$


Multiplying the above equation from right by $\bar{\psi}$ and using Equation (349), we find the proper momentum

$$
p=m u \text {. }
$$

This equation gives the energy of a free particle or electron. For an antiparticle or a positron the spin is negative and the wave function in Equation (354) becomes

$$
\psi=\rho^{1 / 2} e^{\mathbf{i} \varepsilon / 2} \Lambda_{0} e^{-\mathbf{p} \cdot \mathbf{x} / 2 S} .
$$

Thus the wave functions for particle and antiparticle are differentiated by the orientation of spin. In other words the electron and positron wave functions differ by ascribing the senses of local spatial rotation and assigning a value 0 or $\pi$ to the charge conjugation parameter $\varepsilon$.

The wave functions of mass less particles are quite important in the theory of weak interactions. We can decompose the wave function $\psi$ into orthogonal components by multiplying it by the idempotents defined in Equations (62) and (63).

$$
\psi_{ \pm}=\psi \mathcal{J}_{ \pm}
$$

Then the wave function can be expressed as a sum of $\psi_{+}$and $\psi_{-}$.

$$
\psi=\psi_{+}+\psi_{-}
$$

The bivector spin current density is defined as

$$
\frac{\hbar}{2} \psi_{ \pm} c \mathbf{i} \boldsymbol{\sigma}_{3} \bar{\psi}_{ \pm}=\frac{\hbar}{2} \psi c \gamma_{2} \gamma_{1} \bar{\psi}=\rho c S
$$

Therefore, the spin is an independent degree of freedom. The functions $\psi_{+}$and $\psi_{-}$represent the left and right handed wave functions. The zero mass free particle Dirac equation is then given by replacing the wave function $\psi$ with the orthogonal components and setting $m=0$ in the Dirac equation Equation (345).

$$
\partial \psi_{ \pm} 2 S=0
$$

Substituting the plane wave solution of wave function in the above equation gives

$$
p \psi_{ \pm}=0
$$

Squaring $p \psi_{ \pm}$gives $p^{2} \psi_{ \pm} \bar{\psi}_{ \pm}=0$ or $p^{2}=0$. The momentum value in the above equation corresponds to a state with a well defined spin state. The theory is quite applicable to particles like neutrinos.

\subsubsection{The Particle Oscillator in the Complex Vector Space}

Complex vector approach of harmonic oscillator for spin half particles was previously developed by the author [12]. In this section, the harmonic oscillator problem is extended to spin 0,1 and $m / 2$. From the concepts discussed in the above sections, we discern that an elementary particle immersed in zeropoint field may be considered as an oscillator and such an oscillator in complex vector space executes complex rotations with internal radius of rotation $\boldsymbol{\xi}$ and momentum $\boldsymbol{\pi}$. The average values 
of these internal parameters correspond to the deviations in the position and momentum of the particle. Then the Hamiltonian of such the harmonic oscillator in the rest frame of the particle can be expressed as

$$
H=\frac{m \omega_{0}^{2} \xi^{2}}{2}+\frac{\pi^{2}}{2 m}=\frac{\omega_{0}}{2}\left(m \omega_{0} \xi^{2}+\frac{\pi^{2}}{m \omega_{0}}\right),
$$

where $\omega_{0}$ is the characteristic angular frequency of oscillations around a center of point or center of mass point of the particle and $m$ is the mass of the particle. Let us consider a parameter $z$ defined by

$$
z^{2}=\frac{H}{\hbar \omega_{0}}=\frac{1}{2 \hbar}\left(m \omega_{0} \xi^{2}+\frac{\pi^{2}}{m \omega_{0}}\right)=k^{2}\left(a^{2}+b^{2}\right),
$$

where, $k^{2}=1 / 2 \hbar, a^{2}=m \omega_{0} \xi^{2}$ and $b^{2}=\pi^{2} / m \omega_{0}$. Now, the parameter $z$ can be written as a complex number.

$$
z=k(a+i b)
$$

The product $z z^{*}=z^{2}, z^{*}$ being the complex conjugate. The complex numbers correspond to even multivectors in the geometric algebra of Eucledian space and in general the unit imaginary is replaced by a bivector i $\sigma_{3}=\sigma_{1} \sigma_{2}$.

$$
z=k\left(a+\mathbf{i} \sigma_{3} b\right)
$$

When $a$ and $b$ are considered as pure scalars, the even multivecrtor $z$ can be written in a parametric from of a rotor representing rotation in $\mathrm{i} \sigma_{3}$ plane.

$$
z=k r\left(\cos \theta+\mathbf{i} \boldsymbol{\sigma}_{3} \sin \theta\right)=k r \exp \left(\mathbf{i} \boldsymbol{\sigma}_{3} \theta\right),
$$

where the scalars $a$ and $b$ are expressed as $a=r \cos \theta$ and $b=r \sin \theta$. The above equation shows that the oscillations of the particle are equivalent to local complex rotations. A reversion operation on $z$ gives $\bar{z}$.

$$
\bar{z}=k r \exp \left(-\mathbf{i} \boldsymbol{\sigma}_{3} \theta\right)
$$

The product $z \bar{z}$ then gives the energy of the oscillator. However, if the constants $a$ and $b$ were treated as operators just like in quantum mechanics, the product would have contained an additional term i $\sigma_{3}(a b-b a)$ and using the commutation relation $(a b-b a)=i \hbar$ one can easily arrive at the energy of the harmonic oscillator. However, the connection of particle spin to the zero point fields is masked by the quantum mechanical commutation relation. It has been shown in the previous sections that the existence of unit imaginary corresponds to the particle spin. To reveal spin connection of the harmonic oscillator, the particle internal harmonic oscillator is analyzed in the complex vector formalism and this method is purely classical that is without using any quantum commutation relations. This treatment not only gives the correct energy of the oscillator but also elucidates the connection of spin to the particle mass.

Since the internal radius of rotation and momentum of the oscillating particle are basically vectors, one must treat the even multivector $z$ as a complex vector rather than a simple complex number and this can be done by utilizing the property of pseudoscalar $\mathbf{i}^{2}=-1$ and replacing the unit imaginary in Equation (365) by the pseudo scalar $\mathbf{i}$ and expressing $a$ and $b$ as vectors. Then the complex vector $Z$ and its conjugate $\bar{Z}$ can be defined as

$$
Z=k(\mathbf{a}+\mathbf{i} \mathbf{b})
$$




$$
\bar{Z}=k(\mathbf{a}-\mathbf{i} \mathbf{b})
$$

where the vectors $\mathbf{a}$ and $\mathbf{b}$ are defined as

$$
\mathbf{a}=\left(m \omega_{0}\right)^{1 / 2} \boldsymbol{\xi} \quad \text { and } \quad \mathbf{b}=\left(m \omega_{0}\right)^{-1 / 2} \boldsymbol{\pi}
$$

As the pseudoscalar i commutes with all vectors in three dimensional space, we have

$$
\bar{Z} Z=k^{2}\left(a^{2}+b^{2}\right)+2 k^{2} \mathbf{i}(\mathbf{a} \wedge \mathbf{b})
$$

The product $\bar{Z} Z$ is seen to be a multivector containing scalar and vector parts. The scalar part $k^{2}\left(a^{2}+b^{2}\right)$ is equal to $H / \hbar \omega_{0}$. The bivector product, $\mathbf{a} \wedge \mathbf{b}=\boldsymbol{\xi} \wedge \boldsymbol{\pi}$ is the internal zeropoint angular momentum of the particle and represents the spin bivector $S$. In quantum mechanics, conventionally the quantization axis is chosen along $\mathrm{z}$-axis and hence one can choose the spin vector along $\sigma_{3}$ or the spin bivector along $\mathrm{i} \sigma_{3}$ direction. The particle spin bivector is given by Equation (317) and substituting it in Equation (372) gives

$$
\bar{Z} Z=\frac{H}{\hbar \omega_{0}}+\frac{1}{\hbar} \mathbf{i} S=\frac{H}{\hbar \omega_{0}}-\frac{1}{2} \boldsymbol{\sigma}_{3}
$$

Similarly, one can express the product $Z \bar{Z}$ as

$$
Z \bar{Z}=\frac{H}{\hbar \omega_{0}}-\frac{1}{\hbar} \mathbf{i} S=\frac{H}{\hbar \omega_{0}}+\frac{1}{2} \boldsymbol{\sigma}_{3}
$$

Adding Equations (373) and (374), the scalar product of the complex vectors $Z$ and $\bar{Z}$ can be obtained as

$$
Z . \bar{Z}=\frac{1}{2}(Z \bar{Z}+\bar{Z} Z)=\frac{H}{\hbar \omega_{0}} .
$$

Subtracting Equations (373) and (374), we find the relation

$$
(Z \bar{Z}-\bar{Z} Z)=\sigma_{3}
$$

The unit vector $\boldsymbol{\sigma}_{3}$ acts like an operator on the idempotents $\mathcal{J}_{+}$and $\mathcal{J}_{-}$given in Equations (62) and (63) and produces the eigen values $\lambda= \pm 1$.

$$
\sigma_{3} \mathcal{J}_{ \pm}=\lambda \mathcal{J}_{ \pm}
$$

Then multiplying Equation (376) from right by the idempotent $\mathcal{J}_{+}$yields spin-up state of the particle.

$$
(Z \bar{Z}-\bar{Z} Z) \mathcal{J}_{+}=+1 \mathcal{J}_{+}
$$

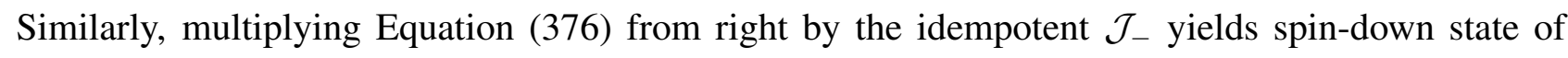
the particle.

$$
(Z \bar{Z}-\bar{Z} Z) \mathcal{J}_{-}=-1 \mathcal{J}_{-}
$$

Further, multiplying Equations (373) and (374) from right by the idempotent $\mathcal{J}_{+}$gives

$$
\bar{Z} Z \mathcal{J}_{+}=\left(\frac{H}{\hbar \omega_{0}}-\frac{1}{2}\right) \mathcal{J}_{+},
$$




$$
Z \bar{Z} \mathcal{J}_{+}=\left(\frac{H}{\hbar \omega_{0}}+\frac{1}{2}\right) \mathcal{J}_{+}
$$

When the particle oscillations and the fluctuations of the zeropoint field are in equilibrium, the oscillations are the simple harmonic oscillations at resonance. Since all these oscillations are at random because of the random fluctuations of the zeropoint field, we consider stochastic average of all such oscillations. In the previous sections, the internal particle structure has been considered with centre of charge and centre of mass points separated by the average radius of rotation. This radius of rotation is considered as a vector $\boldsymbol{\xi}$ which is perpendicular to the momentum vector $\boldsymbol{\pi}$. In the ground state energy of the particle, the motion may be considered as a circular motion with constant magnitude $|\boldsymbol{\xi}|$. In this special case of minimum steady state energy or ground state energy of the harmonic oscillator, the kinetic and potential energies are equal or the total energy is twice the kinetic energy and from the definition of constants $a$ and $b$ in Equation (369), we find $a^{2}=b^{2}$. Now, it can be shown that $Z^{2}=\bar{Z}^{2}=0$. Then the complex vectors $Z$ and $\bar{Z}$ are complex null vectors and expressed as

$$
\begin{aligned}
& Z=k a\left(\boldsymbol{\sigma}_{a}+\mathbf{i} \boldsymbol{\sigma}_{b}\right), \\
& \bar{Z}=k a\left(\boldsymbol{\sigma}_{a}-\mathbf{i} \boldsymbol{\sigma}_{b}\right),
\end{aligned}
$$

where $\sigma_{a}$ and $\sigma_{b}$ are unit vectors along a and $\mathbf{b}$. Since we have chosen the unit vector $\sigma_{3}$ normal to the bivector $\boldsymbol{\sigma}_{a} \boldsymbol{\sigma}_{b}$, Equations (382) and (383) can be written as

$$
\begin{aligned}
& Z=k a \boldsymbol{\sigma}_{a}\left(1-\boldsymbol{\sigma}_{3}\right), \\
& \bar{Z}=k a \boldsymbol{\sigma}_{a}\left(1+\boldsymbol{\sigma}_{3}\right) .
\end{aligned}
$$

Now, the products $Z \bar{Z}$ and $\bar{Z} Z$ are expressed as

$$
\begin{aligned}
& Z \bar{Z}=2 k^{2} a^{2}\left(1+\boldsymbol{\sigma}_{3}\right), \\
& \bar{Z} Z=2 k^{2} a^{2}\left(1-\boldsymbol{\sigma}_{3}\right) .
\end{aligned}
$$

Since, $4 k^{2} \mathbf{a b}=\mathbf{i} \sigma_{3}$, in the case of ground state of the oscillator, the magnitude $4 k^{2} a^{2}=1$. Multiplying the products in the Equations (386) and (387) from right by the idempotent $\mathcal{J}_{+}$gives

$$
\begin{gathered}
Z \bar{Z} \mathcal{J}_{+}=1 \mathcal{J}_{+}, \\
\bar{Z} Z \mathcal{J}_{+}=0 .
\end{gathered}
$$

These relations combined with Equations (380) and (381) directly yield the ground state energy $E_{0}$ of the oscillator per mode.

$$
E_{0}=\frac{\hbar \omega_{0}}{2}
$$

The origin of zeropoint energy is presumed to be due to quantum mechanical effect and considered to be uniformly present throughout space in the form of randomly fluctuating electromagnetic zeropoint fields. In stochastic electrodynamics, Marshall [49] showed that for a harmonic oscillator, the fluctuations produced by zeropoint fields on the particle are exactly in agreement with quantum theory. Thus the ground state energy corresponds to the zeropoint energy of the oscillator per mode [50] 
and an average value is obtained by taking a stochastic average of all such modes. In general the frequency of oscillations of the particle may not necessarily be at resonance with the fluctuations of the spectrum of random zeropoint fields and in such cases one may take into account the higher energy states of the particle oscillator. The random zeropoint field in interaction with the particle oscillator would be expected to produce random displacements producing a shift in the average energy minimum without any change in the particle spin. In the case of higher energy states of the oscillator, the magnitudes of vectors $\mathbf{a}$ and $\mathbf{b}$ are not equal and there is always a possibility of frequencies $\omega_{n}=n \omega_{0}$ with which the particle may oscillate. The particle oscillator may acquire additional energy from the spectrum of zeropoint fields and the Hamiltonian contains additional energy terms. Now, generalizing the Hamiltonian with the condition $\left(H / \hbar \omega_{0}\right) \geq 1 / 2$, suppose that $H=H^{\prime}+\hbar \omega_{0}$. Then the Equations (380) and (381) can be expressed as

$$
\begin{aligned}
& \bar{Z} Z \mathcal{J}_{+}=\left(\frac{H^{\prime}}{\hbar \omega_{0}}+\frac{1}{2}\right) \mathcal{J}_{+}, \\
& Z \bar{Z} \mathcal{J}_{+}=\left(\frac{H^{\prime}}{\hbar \omega_{0}}+\frac{3}{2}\right) \mathcal{J}_{+} .
\end{aligned}
$$

These equations satisfy the relation $(Z \bar{Z}-\bar{Z} Z) \mathcal{J}_{+}=+1 \mathcal{J}_{+}$. Similarly this relation is satisfied in all the cases when $H=H^{\prime \prime}+2 \hbar \omega_{0}, H=H^{\prime \prime \prime}+3 \hbar \omega_{0}$ etc. Continuing the same procedure until the energy reaches its minimum value $H=H_{0}+n \hbar \omega_{0}$ one can find that in each case the relation $(Z \bar{Z}-\bar{Z} Z) \mathcal{J}_{+}=$ $+1 \mathcal{J}_{+}$is satisfied. Thus the argument yields that the terms in brackets of Equations (380) and (381) must be integers. With this correspondence the geometric products $\bar{Z} Z$ and $Z \bar{Z}$ are expressed as

$$
\begin{gathered}
\bar{Z} Z \mathcal{J}_{+}=n \mathcal{J}_{+}, \\
Z \bar{Z} \mathcal{J}_{+}=(n+1) \mathcal{J}_{+} .
\end{gathered}
$$

Comparing these equations with Equations (380) and (381) and replacing $H$ by the energy $E_{n}$ of the oscillator we find

$$
\begin{gathered}
n=\frac{E_{n}}{\omega_{0}}-\frac{1}{2}, \\
n+1=\frac{E_{n}}{\omega_{0}}+\frac{1}{2} .
\end{gathered}
$$

Both these equations finally give the required result for the energy of the harmonic oscillator.

$$
E_{n}=\left(n+\frac{1}{2}\right) \hbar \omega_{0}
$$

The above treatment gives the energy of the oscillator purely on classical grounds.

Choosing the unit vectors $\boldsymbol{\sigma}_{a}$ and $\boldsymbol{\sigma}_{b}$ along $\mathbf{a}$ and $\mathrm{b}$ as above and assuming the unit vector $\boldsymbol{\sigma}_{3}$ normal to the plane $\boldsymbol{\sigma}_{a} \boldsymbol{\sigma}_{b}$, in the general case the complex vectors $Z$ and $\bar{Z}$ can be expressed as

$$
\begin{aligned}
& Z=k \boldsymbol{\sigma}_{a}\left(a-b \boldsymbol{\sigma}_{3}\right), \\
& \bar{Z}=k \boldsymbol{\sigma}_{a}\left(a+b \boldsymbol{\sigma}_{3}\right) .
\end{aligned}
$$

Since the unit vector $\sigma_{3}$ is absorbed by the idempotent $\mathcal{J}_{+}$, multiplying $Z$ and $\bar{Z}$ from right by $\mathcal{J}_{+}$gives

$$
Z \mathcal{J}_{+}=k(a-b) \boldsymbol{\sigma}_{a} \mathcal{J}_{+},
$$




$$
\bar{Z} \mathcal{J}_{+}=k(a+b) \boldsymbol{\sigma}_{a} \mathcal{J}_{+}
$$

Now, one can easily verify the following relations.

$$
\begin{aligned}
& Z \bar{Z} \mathcal{J}_{+}=k^{2}(a-b)^{2} \mathcal{J}_{+} \\
& \bar{Z} Z \mathcal{J}_{+}=k^{2}(a+b)^{2} \mathcal{J}_{+}
\end{aligned}
$$

Then using Equations (393) and (394), we find

$$
\begin{gathered}
k(a-b)=\sqrt{n}, \\
k(a+b)=\sqrt{n+1} .
\end{gathered}
$$

Now, the relations in Equations (400) and (401) are written as

$$
\begin{gathered}
Z \mathcal{J}_{+}=\sqrt{n} \boldsymbol{\sigma}_{a} \mathcal{J}_{+}, \\
\bar{Z} \mathcal{J}_{+}=\sqrt{n+1} \boldsymbol{\sigma}_{a} \mathcal{J}_{+} .
\end{gathered}
$$

From the relations in Equations (404) and (405) we have

$$
2 k a=\sqrt{n+1}+\sqrt{n} ; \quad 2 k b=\sqrt{n+1}-\sqrt{n} ; \quad 4 k^{2} a b=1 .
$$

Thus for higher energies of the oscillator the magnitude of $a$ increases with corresponding decrease in the magnitude of $b$. Since $4 k^{2} a b=1$, the spin remains constant with its magnitude $a b=\hbar / 2$. From Equations (400) and (401), it is straightforward to show

$$
\bar{Z}^{2 l} \mathcal{J}_{+}=Z^{2 l} \mathcal{J}_{+}=[n(n+1)]^{l / 2} \mathcal{J}_{+} \quad \text { for } \quad(l=1,2,3 \ldots) .
$$

If we represent $Z \bar{Z}$ as an operator $N$, we have $N \mathcal{J}_{+}=n \mathcal{J}_{+}$, then the following relations can be easily proved.

$$
\begin{gathered}
N \bar{Z} \mathcal{J}_{+}=\bar{Z}(n+1) \mathcal{J}_{+} \\
N Z \mathcal{J}_{+}=(Z \bar{Z}-1) Z \mathcal{J}_{+}=Z(n-1) \mathcal{J}_{+}
\end{gathered}
$$

Then the following commutation relations are evident.

$$
\begin{gathered}
{[N, \bar{Z}] \mathcal{J}_{+}=\bar{Z} \mathcal{J}_{+}} \\
{[N, Z] \mathcal{J}_{+}=-Z \mathcal{J}_{+}}
\end{gathered}
$$

In the case of ground state of the oscillator, the commutation relations in the above equation reduce to

$$
\begin{aligned}
& N \bar{Z} \mathcal{J}_{+}=(+1) \mathcal{J}_{+}, \\
& N Z \mathcal{J}_{+}=(-1) \mathcal{J}_{+} .
\end{aligned}
$$

These relations and Equations (412) and (413) reveal the fact that the complex vectors $\bar{Z}$ and $Z$ are analogous to the creation and annihilation operators in quantum mechanics and satisfy similar commutation relations. If we work with the idempotent $\mathcal{J}_{-}$the complex vectors reverse their 
actions. The above analysis elucidates the correspondence between the complex vector formalism of harmonic oscillator to the quantum oscillator through equivalence between commutator product and bivector product.

For particles having spin one, like photons spin $|s|=\hbar$ and the harmonic oscillator problem gives the photon energy given by Equation (240). Suppose if we assume $|s|=0$, we have $Z \bar{Z}-\bar{Z} Z=0$. Then either $a$ or $b$ must be zero and the oscillator ceases to oscillate. It means the commutator relation eliminates zeropoint energy. Further, we are free to choose any value for the magnitude of spin. Then we finally arrive at continuous energy spectrum of the oscillator just like a classical oscillator. Thus we find that the existence of spin transforms a classical oscillator into a quantum oscillator.

For mathematical interest, the above procedure may be generalised if we assume the magnitude of spin $|s|=m \hbar / 2$ for $m=1,2, \ldots$. Then by substituting $\mathbf{s}=(m \hbar / 2) \boldsymbol{\sigma}_{3}$ in Equations (373) and (374) we find

$$
\begin{aligned}
& \bar{Z} Z=\left(\frac{H}{\hbar \omega_{0}}-\frac{m \boldsymbol{\sigma}_{3}}{2}\right), \\
& Z \bar{Z}=\left(\frac{H}{\hbar \omega_{0}}+\frac{m \boldsymbol{\sigma}_{3}}{2}\right) .
\end{aligned}
$$

The scalar product of $Z$ and $\bar{Z}$ is obtained by adding Equations (416) and (417).

$$
Z . \bar{Z}=\frac{H}{\hbar \omega_{0}}
$$

This gives the energy of the oscillator. Subtracting Equations (416) and (417) gives the relation

$$
Z \bar{Z}-\bar{Z} Z=m \boldsymbol{\sigma}_{3}
$$

Multiplying the above equation with the idempotent $\mathcal{J}_{+}$, we have

$$
(Z \bar{Z}-\bar{Z} Z) \mathcal{J}_{+}=m \mathcal{J}_{+}
$$

Considering the arguments as above, from this equation one can arrive at the following relations.

$$
\begin{gathered}
Z \bar{Z} \mathcal{J}_{+}=(n+m) \mathcal{J}_{+}, \\
\bar{Z} Z \mathcal{J}_{+}=n \mathcal{J}_{+} .
\end{gathered}
$$

Substituting these relations in Equations (416) and (417) respectively gives the energy of the particle oscillator with spin $m \hbar / 2$.

$$
E_{n}=\left(n+\frac{m}{2}\right) \hbar \omega_{0}
$$

The ground state energy of the oscillator is obtained when $a^{2}=b^{2}$ and in this case the Equations (421) and (422) are expressed as

$$
\begin{gathered}
Z \bar{Z} \mathcal{J}_{+}=m \mathcal{J}_{+}, \\
\bar{Z} Z \mathcal{J}_{+}=0 .
\end{gathered}
$$

The ground state energy or the zeropoint energy per mode of the oscillating particle with spin magnitude $|s|=m \hbar / 2$ is then obtained as

$$
E_{0}=m \hbar \omega_{0} / 2
$$


Thus for $m=1$, and $m=2$, this equation gives the zeropoint energy associated with fermions and bosons respectively. As above, if we define $\bar{Z} Z$ as an operator $\mathrm{N}$ then $N \mathcal{J}_{+}=n \mathcal{J}_{+}$and we find

$$
\begin{gathered}
Z \mathcal{J}_{+}=\sqrt{n} \boldsymbol{\sigma}_{a} \mathcal{J}_{+}, \\
\bar{Z} \mathcal{J}_{+}=\sqrt{n+m} \boldsymbol{\sigma}_{a} \mathcal{J}_{+} .
\end{gathered}
$$

And comparing these equations with Equations (400) and (401) gives

$$
2 k a=\sqrt{n+m}+\sqrt{n} ; \quad 2 k b=\sqrt{n+m}-\sqrt{n} ; \quad 4 k^{2} a b=m .
$$

Since $4 k^{2} a b=m$ the spin remains constant with its magnitude $a b=m \hbar / 2$. Now, using Equations (421) and (422) we have

$$
\begin{gathered}
N \bar{Z} \mathcal{J}_{+}=\bar{Z}(n+m) \mathcal{J}_{+} \\
N Z \mathcal{J}_{+}=(Z \bar{Z}-m) Z \mathcal{J}_{+}=Z(n-m) \mathcal{J}_{+}
\end{gathered}
$$

And it is trivial to find the following commutator relations

$$
\begin{aligned}
& {[N, \bar{Z}] \mathcal{J}_{+}=+m \bar{Z} \mathcal{J}_{+}} \\
& {[N, Z] \mathcal{J}_{+}=-m Z \mathcal{J}_{+}}
\end{aligned}
$$

In the case of ground state of the oscillator, the above relations reduce to

$$
\begin{aligned}
& N \bar{Z} \mathcal{J}_{+}=+m \bar{Z} \mathcal{J}_{+} \\
& N Z \mathcal{J}_{+}=-m Z \mathcal{J}_{+}
\end{aligned}
$$

Now, it may be understood that one particle with spin $m \hbar / 2$ is created by raising $m$ number of spin-half particles and one particle with spin $m \hbar / 2$ is annihilated by lowering $m$ number of spin-half particles.

\section{Summary}

The complex vector is defined as a sum of a vector and a bivector, and found to be a natural extension of a vector. These complex vectors have certain special geometric properties and considered as algebraic entities that represent rotations in space along with specified orientation and direction in space. The complex vectors $Z$ and its conjugate $\bar{Z}$ form a physical space. The set of elements $\left\{1, \boldsymbol{\sigma}_{k} ; k=1,2,3\right\}$ defined from $Z$ and $\bar{Z}$ form a closed complex four dimensional linear space. The set of elements $\left\{1, \boldsymbol{\sigma}_{1}\right\}$ form geometric algebra $\mathcal{G}(1)$. Complexifying $\mathcal{G}(1)$ generates geometric algebra in two dimensions. The set of elements $\left\{1, \boldsymbol{\sigma}_{1}, \boldsymbol{\sigma}_{2}, i\right\}$ form geometric algebra $\mathcal{G}(2)$ and the number of elements are $2^{2}$. The elements of even subalgebra of $\mathcal{G}(2)$ are called complex scalars and unit complex scalars represent rotors and they form rotation group $S O(2)$. The geometric algebra $\mathcal{G}(3)$ can be generated from complexification of $\mathcal{G}(2)$. The set of elements $\left\{1, \boldsymbol{\sigma}_{k}, B_{k}, \mathbf{i}\right\}$ form geometric algebra $\mathcal{G}(3)$ of three dimensional space and the number of elements are $2^{3}$. The general elements of geometric algebra are known as multivectors. The even multivectors represent rotation followed by dilation and 
form spinor algebra. The spinor algebra is very much useful in the formulation of Dirac theory and rigid body dynamics in classical mechanics. A multivector can be decomposed into a complex scalar and a complex vector. The complex scalars form subalgebra of $\mathcal{G}(3)$ under multiplication. The geometric product of two complex vectors is a multivector. The product of unit complex vector with its conjugate gives an idempotent. In conventional quantum mechanics, idempotents correspond to helicity states. A quaternion in geometric algebra is an even multivector and a normalized quaternion represents spatial rotation. The sphere of unit quaternions is a twofold covering group of $S O(3)$ and hence unit quaternions represent the group $S U(2)$. A quaternion in its spinorial form represents a complementary description of spin wave function for fermions. The complexification process in complex vector space allows the generation of higher $n$ dimensional geometric algebra $\mathcal{G}(n)$ from $(n-1)$ dimensional algebra $\mathcal{G}(n-1)$ by considering the unit pseudoscalar $\mathbf{i}_{n}$ identification with square root of minus one. The spacetime algebra of four dimensional spacetime can be generated from the geometric algebra $\mathcal{G}(3)$ by considering a vector $\boldsymbol{\sigma}_{0}=\sqrt{+1}$. An active Lorentz transformation of this vector $\boldsymbol{\sigma}_{0}$ gives the unit vector along the future light cone. A complex vector in spacetime is equal to a sum of relative vector and spatial bivector. The space spanned by $\boldsymbol{\sigma}_{1}$ and $\boldsymbol{\sigma}_{2}$ unit vectors is flat space and considering the unit vector $\gamma_{0}$ normal to the flat space forms a three dimensional spacetime $\mathcal{G}(1,2)$. An even multivector in $\mathcal{G}(1,2)$ represents Lorentz rotation. The three dimensional spacetime gives a natural alternative to the Minkowski spacetime. Considering the unit vector $\gamma_{0}$ normal to the unit vector $\boldsymbol{\sigma}_{1}$ forms two dimensional spacetime. The two dimensional spacetime is quite useful in the geometric representation of spacetime events. The fourth dimension time may be purely obtained from the scalar product of complex vectors or from the product of basis bivectors and can be represented by a vector $\boldsymbol{\sigma}_{0}$. A general element in Clifford algebra is called a paravector and equal to a sum of a scalar and a vector. Since, the product of a complex vector with its conjugate contains a scalar and vector parts, a paravector can also be defined from this product. The paravector space provides required representation of spacetime with some additional set of rules. The geometric algebra of higher dimensional space is generated from the identification of the corresponding pseudoscalar to the square root of minus one in the case of odd space dimensions and to the square root of plus one in the case of even space dimensions $(n>2)$.

Any undamped elliptic harmonic wave can be represented by a product of complex vector and an exponential factor. The complex vector represents a train of plane waves of elliptic harmonic motion. The elliptic harmonic motion can be better visualized in the complex vector approach and complex vector methods can be easily incorporated into the electromagnetic theory. A simplified form of Maxwell's equations is possible with the definition of complex vector electromagnetic field. Complex vector formalism in electromagnetic theory simplifies the expressions and elucidates geometrical understanding of the basic concepts. In an infinite electromagnetic plane wave the electric and magnetic fields are perpendicular and in a finite transverse extent of an electromagnetic wave, the electromagnetic field lines are closed loops and represent circulating energy flow in addition to the energy flow in the propagation direction. Therefore, the energy of photon contains both kinetic and rotational energy components and which allows us to define photon momentum and velocity complex vectors and the energy is expressed as an even multivector. The circulating energy flow represents photon angular momentum. The multivector energy of photon can be expressed in terms of a rotor. The cause of internal complex rotations may be attributed to the fluctuations of zeropoint field. The complex internal field rotations of photon suggest 
that there exists an internal photon harmonic oscillator and internal spin structure. It has been shown that the spin angular momentum causes the complex vector electromagnetic field to rotate in the $\mathbf{E} \wedge \mathbf{B}$ plane without changing the direction of wave vector $\mathrm{k}$. Whereas, the orbital angular momentum of photon causes the plane having the orientation defined by the bivector $\mathbf{r} \wedge \mathrm{k}$ to rotate without changing the direction of $\mathbf{k}$ and the orientation of the bivector $\mathbf{E} \wedge \mathrm{B}$. The complex internal rotations of photon are analyzed in terms of an internal harmonic oscillator in complex vector formalism pertaining to the internal spin rotations and the ground state energy or zeropoint energy of photon is found to be $\hbar \omega$.

In the zitterbeweging model of electron, the structure of electron may be visualized as a point charge in circular motion with frequency of rotation equal to the zitterbewegung frequency and the average radius of rotation equal to half the Compton wavelength. The cause of such rotations has been found to be due to the very existence of zeropoint field. In this view, the structure of an elementary particle is not definitely a point particle with charge and mass or a spherical rigid body with charge distribution. The internal oscillations of an elementary particle like electron elucidate the fact that the particle possesses an extended internal structure. In the complex vector space such oscillations of the particles are considered as spatial local rotations and the angular momentum corresponding to these rotations is the zeropoint angular momentum or particle spin. In the complex vector algebra, the analysis of particle dynamics reveals the fact that the basic reason for relativistic effects that we observe is due to the internal complex rotations. We have discussed the bivector spin dynamics in complex vector space and derived an expression for proper spin and the spin equation in the presence of constant magnetic field. In the constant magnetic field, the particle contains a very low frequency spin precession in addition to its internal complex rotation. As the particle moves in the zeropoint field, the particle itself induces certain modifications to the field to take place at a lower frequency and the particle motion is visualized as a superposition of internal complex rotations on the translational motion. This gives the genesis of de Broglie relation and the quantum nature of particles in general. The wave nature of particles is found to be associated with the zeropoint field and further the uncertainty principle is merely a consequence of more fundamental property of the particles related to the bivector spin. The existence of internal structure of the particle itself suggests that there exist certain correspondence with quantum operators with the internal parameters. The quantum condition is in fact connected with the particle spin. Considering the internal structure of the particle we have derived the Schrödinger equation and found that the very appearance of Planck's constant is directly related to the particle spin. In the rest frame of the particle the rotation in bivector spin plane is a rotor and considering the relation between particle mass and spin, the Dirac-Hestenes equation is derived in the complex vector algebra. It is clear that the internal spin structure of the particle is hidden in both Schrödinger and Dirac equations and the analysis shows that the classical mechanics combined with zeropoint field leads to quantum mechanics. The particle internal harmonic oscillator has been analyzed in the complex vector formalism. It has been shown that the complex vector and its conjugate of the particle oscillator are analogous to the creation and annihilation operators in quantum mechanics. We have discussed the particle oscillator in the zeropoint field for spin half, spin one and higher order spin. The analysis shows that the existence of spin transforms a classical oscillator into a quantum oscillator. 


\section{Conflicts of Interest}

The author declares no conflict of interest.

\section{References}

1. Gibbs, J.W.; Wilson, E.B. Vector Analysis; Charles Scribner's Sons: New York, NY, USA, 1901.

2. Dirac, P.A.M. Physical Principles of Quantum Mechanics; Clarendon Press: Oxford, UK, 1947.

3. Hestenes, D. Space-Time Algebra; Gordon and Breach: New York, NY, USA, 1966.

4. Hestenes, D. Oersted Medal Lecture 2002: Reforming the Mathematical Language of Physics. Am. J. Phys. 2003, 71, 104-121.

5. Hestenes, D. Spacetime physics with Geometric algebra. Am. J. Phys. 2003, 71, 691-714.

6. Sobczyk, G. New Foundations in Mathematics: The Geometric Concept of Number; Springer: New York, NY, USA, 2013.

7. Hestenes, D. New Foundations for Classical Mechanics; D. Reidel Publishing Co.: Dordrecht, The Netherlands; Boston, MA, USA, 1986.

8. Doran, C.; Lansenby, A. Geometric Algebra for Physicists; Cambridge University Press: Cambridge, UK, 2003.

9. Muller, C. Foundations of the Mathematical Theory of Electromagnetic Waves; Springer: New York, NY, USA, 1969; pp. 339-341.

10. Lindell, I.V. Complex vector algebra in electromagnetics. Int. J. Electr. Eng. Educ. 1983, 20, 33-47.

11. Sobczyk, G. Unitary Geometric Algebra. Adv. Appl. Clifford Algebras 2012, 22, 827-836.

12. Muralidhar, K. Complex Vector Formalism of Harmonic Oscillator in Geometric Algebra: Particle Mass, Spin and Dynamics in Complex Vector Space. Found. Phys. 2014, 44, 266-295.

13. Baylis, W.E.; Cabrera, R.; Keselica, J.D. Quantum/Classical interface: Fermion spin. Adv. Appl. Clifford Algebras. 2010, 20, 517-545.

14. Sobczyk, G. Geometric matrix algebra. Linear Algebra Appl. 2008, 429, 1163-1173.

15. Hestenes, D. Vectors, spinors and complex numbers in classical and quantum physics. Am. J. Phys. 1971, 39, 1013-1027.

16. Vold, T.G. An introduction to geometric algebra with an application in rigid body mechanics. Am. J. Phys. 1993, 61, 491-504.

17. Hestenes, D. Geometry of Dirac theory. In Proceedings of the Symposium on Mathematics of Physical Spacetime, Facultad de Quimica, Universdad National Autonoma de Mexico City, Mexico City, Mexico, 1981; pp. 67-96.

18. Chappell, J.M.; Iqbal, A.; Iannella N.; Abbott, D. Revisiting special relativity: A natural algebraic alternative to Minkowski spacetime. PLoS ONE 2012, 7, e51756.

19. Machicote, J.E.R. Time as a geometrical concept involving angular relations in classical mechanics and quatum mechanics. Found. Phys. 2010, 40, 1744-1778.

20. Ueda, K.; Ishikawa, K.L. Auttoclocks play devil's advocate. Nat. Phys. 2011, 7, 371-372. 
21. Baylis, W.E. Geometry of Paravector Sapce With Applications to Relativistic Physics. In Computational Noncommutative Algebra and Applications, Proceedings of the NATO Advanced Study Institute on Computatoinal Noncommutative Algebra and Applications, Il Ciocco, Italy, 6-19 July 2003; NATO Science Series, Sub Series II; Byrnes, J., Eds; Kulwer Academic: Dordrecht, The Netherlands, 2004; Volume 136, pp. 363-387.

22. Landau, L.D.; Lifshitz, E.M. The Classical Theory of Fields; Pergamon Press: Oxford, UK, 1971.

23. Jackson, J.D. Classical Electrodynamics; Wiley Eastern Limited: New Delhi, India, 1978.

24. Vold, T.G. An introduction to geometric calculus and its application to electrodynamics. Am. J. Phys. 1993, 61, 505-513.

25. Ohanian, H.C. What is spin? Am. J. Phys. 1986, 54, 500-505.

26. Cohen-Tannoudji, C.; Dupont-Roc, J.; Grynberg, G. Photons and Atoms; Weily: New York, NY, USA, 1989.

27. Muralidhar, K. The Structure of Photon in Complex Vector Space. EPJD 2015, submitted.

28. Kobe, D.H. A relativistic Schrodinger-like Equation for a Photon and Its second Quantization. Found. Phys. 1999, 29, 1203-1231.

29. Beth, R.A. Mechanical Detection and Measurment of Angular Momentum of Light. Phys. Rev. 1936, 50, 115-125.

30. Leach, L.; Padgett, M.J.; Barnett, S.M.; Frank-Arnold, S.; Courtial, J. Measuring the orbital angular momentum of a single photon. Phys. Rev. Lett. 2002, 88, 257901, doi:10.1103/PhysRevLett.88.257901.

31. Nienhuis, G.; Allen, L. Paraxial wave optics and harmonic oscillators. Phys. Rev. A 1993, 48, 656-665.

32. Barut, A.O.; Bracken, A.J. Zitterbewegung and the internal geometry of electron. Phys. Rev. D 1981, 23, 2454-2462.

33. Sidharth, B. Revisiting Zitterbewegung. Int. J. Theor. Phys. 2009, 48, 497-506.

34. Barut, A.O.; Zanghi, A.J. Classical model of the Dirac electron. Phys. Rev. Lett. 1984, 52, 2009-2012.

35. Hestenes, D. Mysteries and insights of Dirac theory. Ann. Fond. Louis Broglie 2003, 28, 390-408.

36. Hestenes, D. Zitterbewegung in Radiative Processes. In The Electron; Hestenes, D., Weingartshofer, A., Eds.; Kluwer Academic Publishers: Dordrecht, The Netherlands, 1991; pp. 21-36.

37. Hestenes, D. Zitterbewegung in quantum mechanics. Found. Phys. 2010, 40, 1-54.

38. Doran, C.J.L.; Lansenby, A.N.; Gull, S.F.; Somoro, S.; Challinor, A.D. Spacetime Algebra and Electron Physics. Adv. Imaging Electron Phys. 1996, 95, 271-386.

39. Muralidhar, K. Classical origin of quantum spin. Apeiron 2011, 18, 146-160.

40. Muralidhar, K. The spin bivector and zeropoint energy in geometric algebra. Adv. Stud. Theor. Phys. 2012, 6, 675-686.

41. Weyssenhoff, J.; Raabbe, A. Relativistic dynamics of spin fluids and spin particles. Acta Phys. Pol. 1947, 9, 7-18.

42. Salesi G.; Recami, E. A veleocity field and operator for spinning particles in (nonrelativistic) quantum mechanics. Found. Phys. 1998, 28, 763-773. 
43. Cavalleri, G. $\hbar$ derived from cosmology and origin of special relativity and QM. Nuovo Cimento B 1997, 112, 1193-1205.

44. Bosi, L.; Cavalleri, G.; Barbero, F., Bertazzi, G.; Toni E.; Spavieri, G. Review of stochasitic electrodynamics with and without spin. In Proceedings of the Physical Interpretation of Relativity Theory (PIRT XI), London, UK, 12-15 September 2008.

45. Cavalleri, G.; Barbero, F.; Bertazzi, G.; Cesaroni, E.; Tonni, E.; Bosi, L.; Spavieri, G.; Gillies, G.T. A qualitative assessment of stochastic electrodynamics with spin (SEDS): Physical principles and novel applications. Front. Phys. China 2010, 5, 107-122.

46. Okun, L.B. The concept of mass (mass, energy, relativity). Sov. Phys. Uspekhi 1989, 32, 629-638.

47. Sobczyk, G. Special relativity in complex vector algebra. Available online: http://arxiv.org/ abs/0710.0084 (accessed on 2 January 2011).

48. Hestenes, D. Proper dynamics of rigid point particle. J. Math. Phys. 1974, 15, 1778-1786.

49. Marshall, T.W. Random electrodynamics. Proc. R. Soc. Lond. A 1963, 276, 475-491.

50. Boyer, T.H. Random electrodynamics - The theory of classical electrodynamics with classical electromagnetic zero point radiation. Phys. Rev. D 1975, 11, 790-808.

51. Rueda, A. Behavior of classical particles immersed in electromagnetic zero-pint field. Phys. Rev. A 1981, 23, 2020-2040.

52. De la Peña, L.; Cetto, A.M. The Quantum Dice-An Introduction to Stochastic Electrodynamics. Kluwer Academic Publishers: Dordrecht, The Netherlands, 1996.

53. De la Peña, L.; Cetto, A.M.; Hernandez, A.V. The Emerging Quantum: The Physics Behind Quantum Mechanics; Springer: Cham, Switzerland, 2015.

54. Boyer, T.H. Connection between the adiabatic hypothesis of old quantum theory and classical electrodynamics with classical electromagnetic zero-point radiation. Phys. Rev. A 1978, 18, $1238-1245$.

55. Schrödinger, E. Collected Papers on Wave Mechanics; Blackie and Son Limited: London, $\mathrm{UK}, 1928$.

56. Wigner, E. On the quantum correction for thermodynamic equilibrium. Phys. Rev. 1932, 40, 749-759.

57. Dechoum, K.; Franca, H.M.; Malta, C.P. Classical reinterpretation of the Schrödinger equation according to stochastic electrodynamics. In Gravitation and Cosmology: From Hubble Radius to Planck Scale. Fundamental Theories of Physics; Amoroso, R.L., Hunter, G., Kafatos, M., Vigier, J.P., Eds.; Kluwer Academic Publishers: Dordrecht, The Netherlands, 2002; Volume 126, pp. 393-400,

58. Dechoum, K.; Franca, H.M.; Malta, C.P. Classical aspects of the Pauli-Schrödinger equation. Phys. Lett. A 1998, 248, 93-102.

59. Faria, A.J.; Franka, H.M.; Melta, C.P.; Sponchiado, R.C. The vacuum electromagnetic fields and the Schrödinger equation. Found. Phys. 2007, 37, 1296-1305.

60. Cavalleri, G. Schrödinger's Equation as a Consequence of Zitterbewegung. Lett. Nuovo Cimento 1985, 43, 285-291.

61. Cavalleri, G.; Zecca, A. Interpretation of a Schrödinger-like equation derived from non-Markovian process. Phys. Rev. B 1991, 43, 3223-3227. 
62. Hestenes, D. Spin and uncertainty in the interpretation of quantum mechanics. Am. J. Phys. 1979, 47, 399-415.

63. Boudet, R. Quantum Mechanics in the Geometry of Space-Time Elementary Theory; Babaev, E.; Bremer, M.; Calmet, X.; Di Lodovico, F.; Hoogerland, M.; Overduin, J.; Wang, C. H.-T.; Whitaker, A. Eds.; Springer Briefs in Physics; Springer: Heidelberg, Germany, 2011.

(c) 2015 by the author; licensee MDPI, Basel, Switzerland. This article is an open access article distributed under the terms and conditions of the Creative Commons Attribution license (http://creativecommons.org/licenses/by/4.0/). 